\title{
CONDITIONAL LARGE INITIAL DATA SCATTERING RESULTS FOR THE DIRAC-KLEIN-GORDON SYSTEM
}

\author{
TIMOTHY CANDY and SEBASTIAN HERR \\ Universität Bielefeld, Fakultät für Mathematik, Postfach 100131, 33501 Bielefeld, Germany; \\ email: tcandy@math.uni-bielefeld.de, herr@math.uni-bielefeld.de
}

Received 23 November 2017; accepted 9 May 2018

\begin{abstract}
We consider the global behaviour for large solutions of the Dirac-Klein-Gordon system in critical spaces in dimension $1+3$. In particular, we show that bounded solutions exist globally in time and scatter, provided that a controlling space-time Lebesgue norm is finite. A crucial step is to prove nonlinear estimates that exploit the dichotomy between transversality and null structure, and furthermore involve the controlling norm.
\end{abstract}

2010 Mathematics Subject Classification: 42B37, 35Q41 (primary)

\section{Introduction}

The Dirac-Klein-Gordon system for a spinor $\psi: \mathbb{R}^{1+3} \rightarrow \mathbb{C}^{4}$ and a scalar field $\phi: \mathbb{R}^{1+3} \rightarrow \mathbb{R}$ is given as

$$
\begin{aligned}
-i \gamma^{\mu} \partial_{\mu} \psi+M \psi & =\phi \psi \\
\square \phi+m^{2} \phi & =\bar{\psi} \psi,
\end{aligned}
$$

for the Dirac matrices $\gamma^{\mu} \in \mathbb{C}^{4 \times 4}$, using the summation convention with respect to $\mu=0, \ldots, 3$, where $\partial_{0}=\partial_{t}$ and $\partial_{j}=\partial_{x_{j}}$ for $j=1,2,3$. Further, $m, M>0$ are mass parameters and $\bar{\psi}=\psi^{\dagger} \gamma^{0}$, where $\psi^{\dagger}$ is the conjugate transpose. The system (1) arises as a model for the description of particle interactions in relativistic quantum mechanics; see [2] for more details, and we also refer the reader to [23] for a thorough introduction to Dirac equations. The aim of the present paper is to initiate the study of large dispersive solutions to (1), building on our previous results $[1,6]$ on the initial value problem (1) with small initial data.

(c) The Author(s) 2018. This is an Open Access article, distributed under the terms of the Creative Commons Attribution licence (http://creativecommons.org/licenses/by/4.0/), which permits unrestricted re-use, distribution, and reproduction in any medium, provided the original work is properly cited. 
Recently, global well-posedness and scattering results have been proven for initial data $\left(\psi(0), \phi(0), \partial_{t} \phi(0)\right)$, which are small in spaces close to the critical Sobolev space, which is

$$
L^{2}\left(\mathbb{R}^{3}\right) \times \dot{H}^{1 / 2}\left(\mathbb{R}^{3}\right) \times \dot{H}^{-1 / 2}\left(\mathbb{R}^{3}\right) .
$$

More precisely, in the nonresonant case $2 M>m>0$, [24] proved a small data result in a critical Besov space with angular regularity. In [1] we treated the subcritical Sobolev spaces, and for arbitrary $M, m>0$ we proved this for small initial data in the critical Sobolev space with some small amount of additional angular regularity in [6]. We refer the reader to the introductions of [1, 6] for more details of earlier work.

The key in [1, 6, 24] is the use of the null structure in (1) discovered in [9] and the construction of custom-made function spaces, which allow for global-in-time nonlinear estimates. Here, as the first step towards a better understanding of large solutions to (1), we aim at identifying space-time Lebesgue norms that control the global behaviour of dispersive solutions.

We always assume that the Sobolev regularity is $s_{0} \geqslant 0$, the angular regularity is $\sigma \geqslant 0$, and the masses $M, m>0$ satisfy

$$
\begin{aligned}
\text { either } & 0<s_{0} \ll 1, \sigma=0, \quad \text { and } \quad 2 M>m>0, \\
\text { or } & s_{0}=0, \sigma>0, \quad \text { and } M, m>0 .
\end{aligned}
$$

We take data in the Sobolev space

$$
H_{\sigma}^{s_{0}}\left(\mathbb{R}^{3}\right)=\left(1-\Delta_{\mathbb{S}^{2}}\right)^{-\sigma} H^{s_{0}}\left(\mathbb{R}^{3}\right) \text {, with norm }\|f\|_{H_{\sigma}^{s_{0}}}=\left\|\left(1-\Delta_{\mathbb{S}^{2}}\right)^{\sigma} f\right\|_{H^{s_{0}}} .
$$

Thus in the nonresonant regime, (2), we consider data in the standard Sobolev spaces $H^{s_{0}} \times H^{s_{0}+1 / 2} \times H^{s_{0}-1 / 2}$, while in (3), which includes the resonant regime $0<2 M<m$, we work in the critical spaces with a small amount of angular regularity $H_{\sigma}^{0} \times H_{\sigma}^{1 / 2} \times H_{\sigma}^{-1 / 2}, \sigma>0$. Given an interval $I \subset \mathbb{R}$ and $s \geqslant 0$, we define the dispersive type norm $\|u\|_{\mathbf{D}_{0}^{s}(I)}=\left\|\langle\nabla\rangle^{s} u\right\|_{L_{t, x}^{4}\left(I \times \mathbb{R}^{3}\right)}$, and for $s \geqslant 0, \sigma>0$, we define

$$
\|u\|_{\mathbf{D}_{\sigma}^{s}(I)}=\left(\sum_{N \in 2^{\mathbb{N}}} N^{2 \sigma}\left\|\langle\nabla\rangle^{s} H_{N} u\right\|_{L_{t, x}^{4}\left(I \times \mathbb{R}^{3}\right)}^{2}\right)^{1 / 2},
$$

where $H_{N}$ denotes the projection on angular frequencies of size $N$; see (7) below. Our main result is the following.

THEOREM 1. Let $s_{0}, \sigma \geqslant 0$, and $M, m>0$ satisfy either (2) or (3). Consider any maximal $H_{\sigma}^{s_{0}}$-solution

$$
\begin{aligned}
\psi & \in C_{\mathrm{loc}}\left(I^{*}, H_{\sigma}^{s_{0}}\left(\mathbb{R}^{3}, \mathbb{C}^{4}\right)\right) \quad \text { and } \\
\left(\phi, \partial_{t} \phi\right) & \in C_{\mathrm{loc}}\left(I^{*}, H_{\sigma}^{s_{0}+1 / 2}\left(\mathbb{R}^{3}, \mathbb{R}\right) \times H_{\sigma}^{s_{0}-1 / 2}\left(\mathbb{R}^{3}, \mathbb{R}\right)\right)
\end{aligned}
$$


of (1), which is bounded, that is,

$$
\sup _{t \in I^{*}}\left(\|\psi(t)\|_{H_{\sigma}^{s_{0}}\left(\mathbb{R}^{3}\right)}+\left\|\left(\phi, \partial_{t} \phi\right)(t)\right\|_{H_{\sigma}^{s_{0}+1 / 2} \times H_{\sigma}^{s_{0}-1 / 2}\left(\mathbb{R}^{3}\right)}\right)<+\infty .
$$

If $\|\psi\|_{\mathbf{D}_{\sigma}^{-1 / 2}\left(I^{*}\right)}<+\infty$, then we have $I^{*}=\mathbb{R}$ and $(\psi, \phi)$ scatters to a free solution as $t \rightarrow \pm \infty$.

The norm $\|\cdot\|_{\mathbf{D}_{\sigma}^{-1 / 2}}$ is scaling-critical for (1), and in particular, if $\psi$ is a free solution to the Dirac equation, we have the Strichartz bound $\|\psi\|_{\mathbf{D}_{\sigma}^{-1 / 2}(\mathbb{R})} \lesssim$ $\|\psi(0)\|_{H_{\sigma}^{0}}$. It is important to note that a condition of the form $\|\psi\|_{\mathbf{D}_{\sigma}^{-1 / 2}\left(I^{*}\right)}<\infty$ is necessary to ensure scattering. This follows from the fact that (1) admits global stationary solutions of the form

$$
\psi(t)=e^{i t \omega} \psi_{*}, \quad \phi(t)=\left(m^{2}-\Delta\right)^{-1}\left(\overline{\psi_{*}} \psi_{*}\right), \quad \omega \in(0, M),
$$

where $\psi_{*}: \mathbb{R}^{3} \rightarrow \mathbb{C}^{4}$ is smooth and exponentially decreasing; see [11]. In particular, there exist global solutions to (1), which do not scatter to free solutions as $t \rightarrow \pm \infty$.

In recent years, the notion of type-I and type-II blow-up has played an important role in the study of nonlinear wave equations; see the survey [13] for more details and references. Roughly speaking, a maximal solution is of type-I if its spatial Sobolev norm goes to infinity in finite time, and it is of type-II if the spatial Sobolev norm stays finite, but it does not exist for all times. Thus an alternative phrasing of Theorem 1 is that any type-II blow-up solution $(\psi, \phi)$ of (1) with maximal interval $I^{*}$ must satisfy

$$
\|\psi\|_{\mathbf{D}_{\sigma}^{-1 / 2}\left(I^{*}\right)}=+\infty .
$$

The main technical result behind Theorem 1 is Theorem 4, which gives good control over any (strong) solutions with a small $\mathbf{D}_{\sigma}^{-1 / 2}$ norm. We remark that it is possible to replace the hypothesis $\|\psi\|_{\mathbf{D}_{\sigma}^{-1 / 2}\left(I^{*}\right)}<+\infty$ by $\left\|\left(\phi,\langle\nabla\rangle^{-1} \partial_{t} \phi\right)\right\|_{\mathbf{D}_{\sigma}^{0}\left(I^{*}\right)}<$ $+\infty$, which follows immediately from the statement of Theorem 4 and the proof of Theorem 1 presented in Section 4.

In the case of radial data, Theorem 1 covers the critical regularity case $\psi \in L^{2}$, $\left(\phi, \partial_{t} \phi\right) \in H^{1 / 2} \times H^{-1 / 2}$. Strictly speaking however, as the linear Dirac operator does not preserve radial solutions, it is better to consider the partial wave subspace of the lowest degree. More precisely, we let $\mathcal{H}$ be the collection of spinors $\psi_{0} \in$ $L^{2}\left(\mathbb{R}^{3}, \mathbb{C}^{4}\right)$ of the form

$$
\psi_{0}(x)=\left(\begin{array}{c}
f(|x|)\left(\begin{array}{c}
0 \\
1
\end{array}\right) \\
g(|x|)\left(\begin{array}{c}
\omega_{1}+i \omega_{2} \\
\omega_{3}
\end{array}\right)
\end{array}\right)
$$


with $\omega=x /|x|$, and $f, g \in L^{2}\left([0, \infty), r^{2} d r\right)$. The subspace $\mathcal{H}$ is preserved under the linear Dirac operator [23]. Moreover, a computation shows that the subspace $\mathcal{H}$ is preserved under the nonlinear evolution (1), provided that $\phi(0)$ and $\partial_{t} \phi(0)$ are radial. It is worth noting that there are other partial wave subspaces that remain invariant under the evolution of (1) [23]. However, the class $\mathcal{H}$ is used frequently in the literature, for instance the stationary solutions (5) belong to $\mathcal{H}$.

Applying Theorem 1 to data $\psi(0) \in \mathcal{H}$, and exploiting the conservation of charge, $\|\psi(t)\|_{L_{x}^{2}}$, we can then drop the assumption $\|\psi\|_{L^{\infty}\left(I^{*}, H_{\sigma}^{0}\left(\mathbb{R}^{3}\right)\right)}<+\infty$.

COROllary 1. Let $m, M>0$. Suppose that

$$
\left(\psi(0), \phi(0), \partial_{t} \phi(0)\right) \in L^{2}\left(\mathbb{R}^{3}\right) \times H^{1 / 2}\left(\mathbb{R}^{3}\right) \times H^{-1 / 2}\left(\mathbb{R}^{3}\right)
$$

with $\psi(0) \in \mathcal{H}, \phi(0), \partial_{t} \phi(0)$ radial, and that the corresponding $L^{2}$-maximal solution

$$
\begin{aligned}
\psi & \in C_{\mathrm{loc}}\left(I^{*}, L^{2}\left(\mathbb{R}^{3}, \mathbb{C}^{4}\right)\right) \quad \text { and } \\
\left(\phi, \partial_{t} \phi\right) & \in C_{\mathrm{loc}}\left(I^{*}, H^{1 / 2}\left(\mathbb{R}^{3}, \mathbb{R}\right) \times H^{-1 / 2}\left(\mathbb{R}^{3}, \mathbb{R}\right)\right)
\end{aligned}
$$

of (1) satisfies

$$
\sup _{t \in I^{*}}\left\|\left(\phi, \partial_{t} \phi\right)(t)\right\|_{H^{1 / 2} \times H^{-1 / 2}\left(\mathbb{R}^{3}\right)}<+\infty \quad \text { and } \quad\left\|\langle\nabla\rangle^{-1 / 2} \psi\right\|_{L^{4}\left(I^{*} \times \mathbb{R}^{3}\right)}<+\infty .
$$
$t \in I^{*}$

Then we have $I^{*}=\mathbb{R}$ and $(\phi, \psi)$ scatters to a free solution as $t \rightarrow \pm \infty$.

The main novelty of this paper is a certain refinement of the multilinear estimates from $[1,6]$ in the sense that we allow for small positive powers of suitable $L_{t, x}^{4}$ norms on the right-hand side; see Theorem 2 . This is achieved by exploiting the recent progress on bilinear adjoint Fourier restriction estimates [4]. The particular result from [4] we will exploit here is summarized in Theorem 6. These $L_{t, x}^{4}$ norms have the elementary yet crucial property that they can be made arbitrarily small by shrinking the time interval. This has been used successfully to prove global well-posedness and scattering results for wave and Schrödinger equations with polynomial nonlinearities; see for example, $[3,8,14]$ and the references therein. However, for equations with derivative nonlinearities, such as wave maps or the Maxwell-Klein-Gordon system, this is more difficult to exploit due to the presence of more involved norms. Recently, there has been significant progress, such as $[18,19,21,22]$. Our contribution here is closer in spirit to the controlling norm result in [10] in the context of Schrödinger maps.

The estimates proved in this paper have further applications. In particular, they are applied in [5] to prove scattering results for solutions that approximately satisfy a so-called Majorana condition, which defines an open set of large initial data yielding global solutions that scatter. 
The paper is organized as follows. In Section 2 we introduce notation, which is consistent with our previous work [6]. Further, we define the relevant function spaces and provide some auxiliary results. In Section 3 we prove our main local results, namely Theorems 3 and 4, based on nonlinear estimates in Theorem 2, whose proof relies on the results of the last two sections. In Section 4 we prove our main result of this paper, Theorems 1 and Corollary 1. In Section 5 we introduce further notation and preliminary results, which are important in the remaining sections. Section 6 is devoted to the proof of the crucial multilinear estimates in the subcritical regime, while in Section 7 the critical regime is considered, thereby completing the proof of Theorem 2.

\section{Notation and function spaces}

Let $\mathbb{Z}$ denote the integers and $\mathbb{N}$ the nonnegative integers. Given a function $f \in$ $L_{x}^{1}\left(\mathbb{R}^{3}\right)$, we let $\widehat{f}(\xi)=\int_{\mathbb{R}^{3}} f(x) e^{-i x \cdot \xi} d x$ denote the spatial Fourier transform of $f$. Similarly, for $u \in L_{t, x}^{1}\left(\mathbb{R}^{1+3}\right)$, we take $\widetilde{u}(\tau, \xi)=\int_{\mathbb{R}^{1+3}} u(t, x) e^{-i(t, x) \cdot(\tau, \xi)} d x d t$ to be the space-time Fourier transform of $u$. We extend these transforms to tempered distributions in the usual manner. Let $\rho \in C_{0}^{\infty}(\mathbb{R})$ be a smooth bump function satisfying supp $\rho \subset\left\{\frac{1}{2}<t<2\right\}$ and $\sum_{\lambda \in 2^{\mathbb{Z}}} \rho(t / \lambda)=1$ for $t \neq 0$, and take $\rho_{\leqslant 1}=\sum_{\lambda \leqslant 1} \rho(t / \lambda)$ for $t \neq 0$, and $\rho_{\leqslant 1}(0)=1$. Set $\rho_{\leqslant \lambda}(t)=\rho_{\leqslant 1}(t / \lambda)$ for $\lambda \in 2^{\mathbb{Z}}$. For each $\lambda \in 2^{\mathbb{N}}$ and $d \in 2^{\mathbb{Z}}$ we define the Littlewood-Paley multipliers $P_{\lambda}$, and the modulation localization operators $C_{d}^{ \pm, m}$ as

$$
\begin{aligned}
& \widehat{P_{\lambda} f}(\xi)=\rho\left(\frac{|\xi|}{\lambda}\right) \widehat{f}(\xi) \text { if } \lambda>1, \quad \widehat{P_{1} f}(\xi)=\rho_{\leqslant 1}(|\xi|) \widehat{f}(\xi),
\end{aligned}
$$

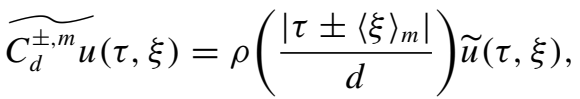

where $\langle\xi\rangle_{m}=\left(m^{2}+|\xi|^{2}\right)^{1 / 2}$. Thus $P_{\lambda}$ localizes to frequencies of size $\lambda$, and $C_{d}^{ \pm, m}$ localizes to space-time frequencies at distance $d$ from the surface $\tau \pm\langle\xi\rangle_{m}=0$. We also define

$$
C_{\leqslant d}^{ \pm, m}=\sum_{d^{\prime} \leqslant d} C_{d^{\prime}}^{ \pm, m}
$$

which localizes to space-time frequencies within $d$ of $\tau \pm\langle\xi\rangle_{m}=0$. We define the localization operators to angular frequencies of size $N \in 2^{\mathbb{N}}$ by

$$
\begin{aligned}
\left(H_{N} f\right)(x) & =\sum_{\ell \in \mathbb{N}} \sum_{n=0}^{2 \ell} \rho\left(\frac{\ell}{N}\right)\left\langle f(|x| \cdot), y_{\ell, n}\right\rangle_{L^{2}\left(\mathbb{S}^{2}\right)} y_{\ell, n}\left(\frac{x}{|x|}\right)(N>1), \\
H_{1} f(x) & =\sum_{\ell \in \mathbb{N}} \sum_{n=0}^{2 \ell} \rho_{\leqslant 1}(\ell)\left\langle f(|x| \cdot), y_{\ell, n}\right\rangle_{L^{2}\left(\mathbb{S}^{2}\right)} y_{\ell, n}\left(\frac{x}{|x|}\right),
\end{aligned}
$$


where $\left(y_{\ell, n}\right)_{n=0, \ldots, 2 \ell}$ denotes an orthonormal basis for the space of homogeneous harmonic polynomials of degree $\ell$, as in [6, Section 7B].

To simplify notation somewhat, in Sections 6 and 7, we use the shorthand

$$
C_{d}^{ \pm}=C_{d}^{ \pm, 1}, \quad \mathcal{C}_{d}^{ \pm}=\Pi_{ \pm} C_{d}^{ \pm, M},
$$

where the projections $\Pi_{ \pm}$are defined as

$$
\Pi_{ \pm}=\frac{1}{2}\left(I \pm \frac{1}{\langle\nabla\rangle_{M}}\left(-i \gamma^{0} \gamma^{j} \partial_{j}+M \gamma^{0}\right)\right) .
$$

The projections $\Pi_{ \pm}$diagonalize the Dirac operator, for instance we have the identity

$$
\left(-i \gamma^{\mu} \partial_{\mu}+M\right) \Pi_{ \pm} \psi=\gamma^{0}\left(-i \partial_{t} \pm\langle\nabla\rangle_{M}\right) \Pi_{ \pm} \psi
$$

and are also used to uncover the null structure hidden in the product $\bar{\psi} \psi$. See Section 5 for further details.

Define the propagator for the homogeneous half-wave equation as $\mathcal{U}_{m}^{ \pm}(t)=$ $e^{\mp i t\langle\nabla\rangle_{m}}$ and let $\mathcal{U}_{M}$ denote the free Dirac propagator. Explicitly we have

$$
\mathcal{U}_{M}(t)=\mathcal{U}_{M}^{+}(t) \Pi_{+}+\mathcal{U}_{M}^{-}(t) \Pi_{-} .
$$

Given $t_{0} \in I \subset \mathbb{R}$ and $F \in L_{t}^{\infty} L_{x}^{2}\left(I \times \mathbb{R}^{3}\right)$, for $t \in I$ we let $\mathcal{I}_{t_{0}}^{ \pm, m}[F](t)$ denote the inhomogeneous solution operator for the half-wave equation

$$
\mathcal{I}_{t_{0}}^{ \pm, m}[F](t)=i \int_{t_{0}}^{t} \mathcal{U}_{m}^{ \pm}\left(t-t^{\prime}\right) F\left(t^{\prime}\right) d t^{\prime}
$$

and $\mathcal{I}_{t_{0}}^{M}[F](t)=\mathcal{I}_{t_{0}}^{+, M}\left[\Pi_{+} F\right](t)+\mathcal{I}_{t_{0}}^{-, M}\left[\Pi_{-} F\right](t)$ denote the inhomogeneous solution operator for the Dirac equation. Thus, if $-i \partial_{t} u+\langle\nabla\rangle_{m} u=F$, then we can write the Duhamel formula as

$$
u(t)=\mathcal{U}_{m}^{+}\left(t-t_{0}\right) u\left(t_{0}\right)+\mathcal{I}_{t_{0}}^{+, m}[F](t) .
$$

Similarly, for the Dirac equation, if $-i \gamma^{\mu} \partial_{\mu} \psi+M \psi=G$, then we have

$$
\psi(t)=\mathcal{U}_{M}\left(t-t_{0}\right) \psi\left(t_{0}\right)+\mathcal{I}_{t_{0}}^{M}\left[\gamma^{0} G\right](t) .
$$

We now define the main function spaces in which we construct solutions. The basic building blocks are the $V^{2}$ spaces introduced by Koch and Tataru [15], and studied systematically in $[12,16]$. Let $\mathcal{Z}=\left\{\left(t_{j}\right)_{j \in \mathbb{Z}} \mid t_{j} \in \mathbb{R}\right.$ and $\left.t_{j}<t_{j+1}\right\}$ and $1 \leqslant p<\infty$. For a function $u: \mathbb{R} \rightarrow L_{x}^{2}$, the $p$-variation of $u$ is defined as

$$
|u|_{V^{p}}=\sup _{\left(t_{j}\right) \in \mathcal{Z}}\left(\sum_{j \in \mathbb{Z}}\left\|u\left(t_{j}\right)-u\left(t_{j-1}\right)\right\|_{L_{x}^{2}}^{p}\right)^{1 / p} .
$$


The normed space $V^{p}$ is then defined to be all right-continuous functions $u: \mathbb{R} \rightarrow$ $L_{x}^{2}\left(\mathbb{R}^{3}\right)$ satisfying

$$
\|u\|_{V^{p}}=\|u\|_{L_{t}^{\infty} L_{x}^{2}}+|u|_{V^{p}}<\infty .
$$

The space $V^{p}$ is complete and functions in $V^{p}$ have one-sided limits at each point, including $\pm \infty$. Define $V_{ \pm, m}^{p}=\mathcal{U}_{m}^{ \pm}(t) V^{p}$ with the norm

$$
\|u\|_{V_{ \pm, m}^{p}}=\left\|\mathcal{U}_{m}^{ \pm}(-t) u\right\|_{V^{p}}
$$

For $m \geqslant 0$ and $2 \leqslant q \leqslant \infty$, and any $d \in 2^{\mathbb{Z}}$ we have

$$
\left\|C_{d}^{ \pm, m} u\right\|_{L_{t}^{q} L_{x}^{2}} \lesssim d^{-1 / q}\|u\|_{V_{ \pm, m}^{2}}
$$

see [12, Corollary 2.18]. We also require an additional auxiliary norm, which is used to obtain a high-low frequency gain in a particular case of the bilinear estimates appearing in Sections 6 and 7. Given $1<a<2$ and $b>0$ we define

$$
\|u\|_{Y_{\lambda}^{ \pm, m}}=\sup _{d \in 2^{\mathbb{Z}}} d^{1 / a}\left(\frac{\min \{d, \lambda\}}{\lambda}\right)^{b}\left\|C_{d}^{ \pm, m} P_{\lambda} u\right\|_{L_{t}^{a} L_{x}^{2}} .
$$

The parameters $a$ and $b$ are fixed later in Sections 6 and 7, but roughly we take $1 / a-\frac{1}{2} \approx \sigma+s_{0}$, and $b \approx 1 / a-\frac{1}{2}$ where $s_{0}$ and $\sigma$ are as in (2) or (3). For $s \in \mathbb{R}$ and $\sigma>0$, we define our main function norms as

$$
\begin{aligned}
\|u\|_{\mathbf{V}_{ \pm, m}^{s, \sigma}} & =\left(\sum_{\lambda, N \in 2^{\mathbb{N}}} \lambda^{2 s} N^{2 \sigma}\left\|P_{\lambda} H_{N} u\right\|_{V_{ \pm, m}^{2}}^{2}\right)^{1 / 2} \\
\|u\|_{\mathbf{Y}_{ \pm, m}^{s, \sigma}} & =\left(\sum_{\lambda, N \in 2^{\mathbb{N}}} \lambda^{2 s} N^{2 \sigma}\left\|H_{N} u\right\|_{Y_{\lambda}^{ \pm, m}}^{2}\right)^{1 / 2}
\end{aligned}
$$

while if $\sigma=0$ we take

$$
\begin{aligned}
\|u\|_{\mathbf{V}_{ \pm, m}^{s, 0}} & =\left(\sum_{\lambda \in 2^{\mathbb{N}}} \lambda^{2 s}\left\|P_{\lambda} u\right\|_{V_{ \pm, m}^{2}}^{2}\right)^{1 / 2} \\
\|u\|_{\mathbf{Y}_{ \pm, m}^{s, 0}} & =\left(\sum_{\lambda \in \mathbb{2}^{\mathbb{N}}} \lambda^{2 s}\|u\|_{Y_{\lambda}^{ \pm, m}}^{2}\right)^{1 / 2}
\end{aligned}
$$

Note that strictly speaking $\|\cdot\|_{\mathbf{Y}_{ \pm, m}^{s, \sigma}}$ is not a norm, as $\left\|\mathcal{U}_{m}^{ \pm}(t) f\right\|_{\mathbf{Y}_{ \pm, m}^{s, \sigma}}=0$ for all $f \in H_{\sigma}^{s}$. We finally define

$$
\|u\|_{\mathbf{F}_{ \pm, m}^{s, \sigma}}=\|u\|_{\mathbf{V}_{ \pm, m}^{s, \sigma}}+\|u\|_{\mathbf{Y}_{ \pm, m}^{s, \sigma}}
$$


The wave component of the DKG equation is estimated in $\mathbf{V}_{ \pm, m}^{s, \sigma}$. On the other hand, to control solutions to the Dirac equation, we define

$$
\|\psi\|_{\mathbf{V}_{M}^{s, \sigma}}=\left\|\Pi_{+} \psi\right\|_{\mathbf{V}_{+, M}^{s, \sigma}}+\left\|\Pi_{-} \psi\right\|_{\mathbf{V}_{-, M}^{s, \sigma}}, \quad\|\psi\|_{\mathbf{Y}_{M}^{s, \sigma}}=\left\|\Pi_{+} \psi\right\|_{\mathbf{Y}_{+, M}^{s, \sigma}}+\left\|\Pi_{-} \psi\right\|_{\mathbf{Y}_{-, M}^{s, \sigma}}
$$

and

$$
\|\psi\|_{\mathbf{F}_{M}^{s, \sigma}}=\|\psi\|_{\mathbf{V}_{M}^{s, \sigma}}+\|\psi\|_{\mathbf{Y}_{M}^{s, \sigma}} .
$$

The Banach space $\mathbf{V}_{ \pm, m}^{s, \sigma}$ is then defined as the collection of all right-continuous functions $u \in L_{t}^{\infty} H_{\sigma}^{s}$ such that $\|u\|_{\mathbf{V}_{ \pm, m}^{s, \sigma}}<\infty$. The Banach spaces $\mathbf{V}_{M}^{s, \sigma}$ and $\mathbf{F}_{M}^{s, \sigma}$ are defined similarly.

We next localize the spaces constructed above to intervals. Let $I \subset \mathbb{R}$ be a leftclosed interval and right-open (in the sequel, left-closed for short) and take $\mathbb{1}_{I}(t)$ to be the corresponding indicator function. Given a function $u$ on $I$, we make a harmless abuse of notation and think of $\mathbb{1}_{I}(t) u(t)$ as a function defined on $\mathbb{R}$. In other words, $\mathbb{1}_{I} u$ is the extension of $u$ by zero to a function on $\mathbb{R}$. We then define $\mathbf{V}_{ \pm, m}^{s, \sigma}(I)$ as the set of all right-continuous functions $u \in L_{t}^{\infty} H_{\sigma}^{s}\left(I \times \mathbb{R}^{3}\right)$ such that $\mathbb{1}_{I} u \in \mathbf{V}_{ \pm, m}^{s, \sigma}$ with the obvious norm

$$
\|u\|_{\mathbf{V}_{ \pm, m}^{s, \sigma}(I)}=\left\|\mathbb{1}_{I} u\right\|_{\mathbf{V}_{ \pm, m}^{s, \sigma}} .
$$

The Banach space $\mathbf{F}_{M}^{s, \sigma}(I)$, and the norms $\|\cdot\|_{\mathbf{F}_{M}^{s, \sigma}(I)}$ and $\|\cdot\|_{\mathbf{Y}_{M}^{s, \sigma}(I)}$ are defined analogously. Note that the existence of left-sided limits in $V^{2}$ immediately implies that if $u \in \mathbf{V}_{ \pm, m}^{s, \sigma}(I)$ then there exists $f \in H_{\sigma}^{s}$ such that $\left\|u(t)-\mathcal{U}_{m}^{ \pm}(t) f\right\|_{H_{\sigma}^{s}} \rightarrow 0$ as $t \rightarrow \sup I$. In particular, if $I=\left[t_{0}, t_{1}\right), t_{1}<\infty$, and $\|u\|_{\mathbf{V}_{ \pm, m}^{s, \sigma}(I)}<\infty$ then $u\left(t_{1}\right) \in H_{\sigma}^{s}$ is well defined.

The following lemma shows that we may freely restrict the spaces to smaller intervals.

LEMMA 1. Let $M>0, s, \sigma \geqslant 0$, and $I$ and $I^{\prime}$ be left-closed intervals with $I \subset I^{\prime}$. If $\psi \in \mathbf{F}_{M}^{s, \sigma}\left(I^{\prime}\right)$ and $\phi \in \mathbf{V}_{+, M}^{s, \sigma}\left(I^{\prime}\right)$, then

$$
\|\psi\|_{\mathbf{F}_{M}^{s, \sigma}(I)} \lesssim\|\psi\|_{\mathbf{F}_{M}^{s, \sigma}\left(I^{\prime}\right)}, \quad\|\phi\|_{\mathbf{V}_{+, M}^{s, \sigma}(I)} \lesssim\|\phi\|_{\mathbf{V}_{+, M}^{s, \sigma}\left(I^{\prime}\right)} .
$$

Proof. By taking differences, exploiting translation invariance, and unpacking the definitions of the spaces $\mathbf{F}_{M}^{s, \sigma}(I)$ and $\mathbf{V}_{+, M}^{s, \sigma}(I)$, it suffices to show that

$$
\left\|\mathbb{1}_{I} u\right\|_{V^{2}} \leqslant 2\|u\|_{V^{2}}
$$

and for any $\lambda \in 2^{\mathbb{N}}$

$$
\left\|\mathbb{1}_{I} u\right\|_{Y_{\lambda}^{ \pm, M}} \lesssim\left\|P_{\lambda} u\right\|_{L_{t}^{\infty} L_{x}^{2}}+\|u\|_{Y_{\lambda}^{ \pm, M}}
$$


with $I=[0, \infty)$. The first inequality follows by noting that for any increasing sequence $\left(t_{j}\right)_{j \in \mathbb{Z}}$ we have

$$
\sum_{j \in \mathbb{Z}}\left\|\left(\mathbb{1}_{I} u\right)\left(t_{j}\right)-\left(\mathbb{1}_{I} u\right)\left(t_{j-1}\right)\right\|_{L_{x}^{2}\left(\mathbb{R}^{3}\right)}^{2} \leqslant\|u\|_{L_{t}^{\infty} L_{x}^{2}}^{2}+|u|_{V_{ \pm, M}^{2}}^{2}=\|u\|_{V_{ \pm, M}^{2}}^{2} .
$$

On the other hand, to prove (10), we start by defining the time frequency localization operators $P_{d}^{(t)}=\rho\left(\left|-i \partial_{t}\right| / d\right)$ and $P_{\leqslant d}^{(t)}=\rho_{\leqslant 1}\left(\left|-i \partial_{t}\right| / d\right)$, where $\rho$ is as in the definition of the $C_{d}^{M, \pm}$ and $P_{\lambda}$ multipliers. Suppose for the moment that we have the bounds

$$
\left\|\mathbb{1}_{I}-P_{\ll d}^{(t)} \mathbb{1}_{I}\right\|_{L_{t}^{a}} \lesssim d^{-1 / a}, \quad\left\|P_{\ll d}^{(t)} \mathbb{1}_{I}\right\|_{L_{t}^{\infty}} \lesssim 1 .
$$

Let $F(t)=\mathcal{U}_{ \pm}^{M}(-t) u$. The identity $C_{d}^{ \pm, M}=\mathcal{U}_{ \pm}^{M}(t) P_{d}^{(t)} \mathcal{U}_{ \pm}^{M}(-t)$, together with (11) and the fact that the free solution propagators are unitary on $L_{x}^{2}\left(\mathbb{R}^{3}\right)$, implies that

$$
\begin{aligned}
\left\|C_{d}^{ \pm, M}\left(\mathbb{1}_{I} u\right)\right\|_{L_{t}^{a} L_{x}^{2}} & =\left\|P_{d}^{(t)}\left(\mathbb{1}_{I} F\right)\right\|_{L_{t}^{a} L_{x}^{2}} \\
& \leqslant\left\|P_{d}^{(t)}\left(\left[\mathbb{1}_{I}-P_{\ll d}^{(t)} \mathbb{1}_{I}\right] F\right)\right\|_{L_{t}^{a} L_{x}^{2}}+\left\|P_{d}^{(t)}\left(P_{\ll d}^{(t)} \mathbb{1}_{I} F\right)\right\|_{L_{t}^{a} L_{x}^{2} .} \\
& \lesssim\left\|\left[\mathbb{1}_{I}-P_{\ll d}^{(t)} \mathbb{1}_{I}\right] F\right\|_{L_{t}^{a} L_{x}^{2}}+\sup _{d^{\prime} \approx d}\left\|P_{\ll d}^{(t)} \mathbb{1}_{I} P_{d^{\prime}}^{(t)} F\right\|_{L_{t}^{a} L_{x}^{2}} \\
& \lesssim d^{-1 / a}\|u\|_{L_{t}^{\infty} L_{x}^{2}}+\sup _{d^{\prime} \approx d}\left\|C_{d^{\prime}}^{ \pm, M} u\right\|_{L_{t}^{a} L_{x}^{2}} .
\end{aligned}
$$

Thus, by definition of the $Y_{\lambda}^{ \pm, M}$ norm, the required bound (10) follows. Consequently, it only remains to prove the bounds (11). To this end, the definition of the $P_{\ll d}^{(t)}$ multipliers implies that there exists a rapidly decreasing function $\sigma \in C^{\infty}(\mathbb{R})$ with $\int_{\mathbb{R}} \sigma(t) d t=1$ such that

$$
P_{\ll d}^{(t)} \mathbb{1}_{I}(t)=\int_{0}^{\infty} \sigma(t d-s) d s .
$$

Hence the second estimate in (11) is immediate. On the other hand, for the first term, we observe that the rapid decay of $\sigma$ gives

$$
\begin{aligned}
\left|\mathbb{1}_{I}(t)-P_{\ll d}^{(t)} \mathbb{1}_{I}(t)\right| & =\left|\mathbb{1}_{I}(t) \int_{\mathbb{R}} \sigma(t d-s) d s-\int_{0}^{\infty} \sigma(t d-s)\right| \\
& \leqslant\left|\int_{\mathbb{R}} \sigma(|t| d+|s|) d s\right|+\left|\int_{\mathbb{R}} \sigma(-|t| d-|s|) d s\right| \\
& \lesssim\langle t d\rangle^{-10}
\end{aligned}
$$

and hence (11) follows. 
A straightforward computation implies that linear solutions belong to the spaces $\mathbf{F}_{M}^{s, \sigma}$ and $\mathbf{V}_{+, m}^{s, \sigma}$. Lemma 1 implies that for any $s, \sigma \geqslant 0$, and $t_{0} \in I \subset \mathbb{R}$ we have

$$
\left\|\mathcal{U}_{M}\left(t-t_{0}\right) \psi_{0}\right\|_{\mathbf{F}_{M}^{s, \sigma}(I)} \lesssim\left\|\psi_{0}\right\|_{H_{\sigma}^{s}}, \quad\left\|\mathcal{U}_{m}^{+}\left(t-t_{0}\right) \phi_{0}\right\|_{\mathbf{V}_{+, m}^{s, \sigma}(I)} \lesssim\left\|\phi_{0}\right\|_{H_{\sigma}^{s}} .
$$

The Strichartz type spaces are also controlled by the $\mathbf{F}_{M}^{s, \sigma}$ and $\mathbf{V}_{+, m}^{s, \sigma}$ norms. We give a more precise version of this statement in Section 5, and for the moment we simply recall that we have the bounds

$$
\begin{aligned}
\|\psi\|_{L_{t}^{\infty} H_{\sigma}^{s}\left(I \times \mathbb{R}^{3}\right)}+\|\psi\|_{\mathbf{D}_{\sigma}^{s-1 / 2}(I)} & \lesssim\|\psi\|_{\mathbf{V}_{M}^{s, \sigma}(I)} \\
\|\phi\|_{L_{t}^{\infty} H_{\sigma}^{s+1 / 2}\left(I \times \mathbb{R}^{3}\right)}+\|\phi\|_{\mathbf{D}_{\sigma}^{s}(I)} & \lesssim\|\phi\|_{\mathbf{V}_{+, m}^{s+1 / 2, \sigma}(I)}
\end{aligned}
$$

These estimates follow directly from the fact that the estimates for the free solutions immediately imply bounds in the corresponding $V^{2}$ space, the details can be found in, for instance, [12]. We also need to understand how the norm $\|\cdot\|_{\mathbf{F}_{M}^{s, \sigma}(I)}$ depends on the interval $I$. Clearly, if $I$ is left-closed, and we can write $I=I_{1} \cup I_{2}$ with $I_{1}, I_{2}$ disjoint left-closed intervals, then by the triangle inequality we have the bound

$$
\begin{aligned}
\|u\|_{\mathbf{V}_{ \pm, m}^{s, \sigma}(I)} & =\left\|\mathbb{1}_{I}(t) u\right\|_{\mathbf{V}_{ \pm, m}^{s, \sigma}} \leqslant\left\|\mathbb{1}_{I_{1}}(t) u\right\|_{\mathbf{V}_{ \pm, m}^{s, \sigma}}+\left\|\mathbb{1}_{I_{2}}(t) u\right\|_{\mathbf{V}_{ \pm, m}^{s, \sigma}} \\
& =\|u\|_{\mathbf{V}_{ \pm, m}^{s, \sigma}\left(I_{1}\right)}+\|u\|_{\mathbf{V}_{ \pm, m}^{s, \sigma}\left(I_{2}\right)} .
\end{aligned}
$$

An identical argument gives

$$
\|u\|_{\mathbf{F}_{M}^{s, \sigma}(I)} \leqslant\|u\|_{\mathbf{F}_{M}^{s, \sigma}\left(I_{1}\right)}+\|u\|_{\mathbf{F}_{M}^{s, \sigma}\left(I_{2}\right)} .
$$

\section{Local theory for the Dirac-Klein-Gordon system}

In this section, we derive two key consequences of the bilinear estimates obtained in Sections 6 and 7, namely Theorems 3 and 4. These theorems show that the time of existence of solutions to the DKG system can be controlled by the $L_{t, x}^{4}$ norm, or, more precisely, by the $\|\cdot\|_{\mathbf{D}_{\sigma}^{0}}$ norms. In particular, we refine the previous local and small data global results obtained in $[1,6]$. The proof of Theorem 1 is then an easy consequence.

We now start with the local theory. By time reversibility, it is enough to consider the forward-in-time problem; thus we always work on left-closed intervals $I=$ $\left[t_{0}, t_{1}\right)$ where potentially we may have $t_{1}=\infty$. In addition, instead of the secondorder system (1), we prefer to work with a first-order system. More precisely, we construct $\psi: I \times \mathbb{R}^{3} \rightarrow \mathbb{C}^{4}$ and $\phi_{+}: I \times \mathbb{R}^{3} \rightarrow \mathbb{C}$ solving the first-order system

$$
\begin{aligned}
-i \gamma^{\mu} \partial_{\mu} \psi+M \psi & =\operatorname{Re}\left(\phi_{+}\right) \psi \\
-i \partial_{t} \phi_{+}+\langle\nabla\rangle_{m} \phi_{+} & =\langle\nabla\rangle_{m}^{-1}(\bar{\psi} \psi) .
\end{aligned}
$$


If we let $\phi=\operatorname{Re}\left(\phi_{+}\right)$, a standard computation using the fact that $\bar{\psi} \psi \in \mathbb{R}$ then shows that, at least for classical solutions, $(\psi, \phi)$ is a solution to the original Dirac-Klein-Gordon system (1).

We next clarify precisely what we mean by solutions and maximal solutions.

DEFINITION 1. Let $s, \sigma \in \mathbb{R}$.

(1) We say $\left(\psi, \phi_{+}\right): I \times \mathbb{R}^{3} \rightarrow \mathbb{C}^{4} \times \mathbb{C}$ is an $H_{\sigma}^{s}$-strong solution on an interval $I \subset \mathbb{R}$, if

$$
\left(\psi, \phi_{+}\right) \in C\left(I, H_{\sigma}^{s}\left(\mathbb{R}^{3}, \mathbb{C}^{4}\right) \times H_{\sigma}^{s+1 / 2}\left(\mathbb{R}^{3}, \mathbb{C}\right)\right)
$$

and there exists a sequence $\left(\psi_{n}, \phi_{n}\right) \in C^{2}\left(I, H^{m}\left(\mathbb{R}^{3}, \mathbb{C}^{4} \times \mathbb{C}\right)\right), m=\max \{10$, $s\}$, of classical solutions to (16) such that, for any compact $I^{\prime} \subseteq I$,

$$
\sup _{t \in I^{\prime}}\left(\left\|\psi(t)-\psi_{n}(t)\right\|_{H_{\sigma}^{s}\left(\mathbb{R}^{3}, \mathbb{C}^{4}\right)}+\left\|\phi_{+}(t)-\phi_{n}(t)\right\|_{H_{\sigma}^{1 / 2+s}\left(\mathbb{R}^{3}, \mathbb{C}\right)}\right) \rightarrow 0 .
$$

(2) We say $\left(\psi, \phi_{+}\right):\left[t_{0}, t^{*}\right) \times \mathbb{R}^{3} \rightarrow \mathbb{C}^{4} \times \mathbb{C}$ is a (forward) maximal $H_{\sigma}^{s}$-solution if the following two properties hold:

(a) for any $t_{1} \in\left(t_{0}, t^{*}\right),\left(\psi, \phi_{+}\right)$is a strong $H_{\sigma}^{s}$-solution on $\left[t_{0}, t_{1}\right)$;

(b) if $\left(\psi^{\prime}, \phi_{+}^{\prime}\right): I \times \mathbb{R}^{3} \rightarrow \mathbb{C}^{4} \times \mathbb{C}$ is a strong $H_{\sigma}^{s}$-solution on an interval $I$ satisfying $I \cap\left[t_{0}, t^{*}\right) \neq \varnothing$ and $\left(\psi^{\prime}, \phi_{+}^{\prime}\right)=\left(\psi, \phi_{+}\right)$on $I \cap\left[t_{0}, t^{*}\right)$, then $I \cap\left[t_{0}, \infty\right) \subseteq\left[t_{0}, t^{*}\right)$.

We remark that $H_{\sigma}^{s}$-strong solutions are unique, owing to the fact that classical solutions are unique and the local Lipschitz continuity of the data-to-solution map in $H_{\sigma}^{s}$ established below. Moreover, the local theory we develop below implies that an $H_{\sigma}^{s_{0}}$-strong solution to (16) on an interval $I$ is also locally in $\mathbf{F}_{M}^{s_{0}, \sigma} \times \mathbf{V}_{+, m}^{s_{0}+1 / 2, \sigma}$. Furthermore, we also show that if we have control over both the $L_{t, x}^{4}$ type norm $\|\psi\|_{\mathbf{D}_{\sigma(I)}^{0}}$ and the data norm $\left\|\left(\psi, \phi_{+}\right)\left(t_{0}\right)\right\|_{H_{\sigma}^{s_{0}} \times H_{\sigma}^{s_{0}+1 / 2}}$, then in fact $\left(\psi, \phi_{+}\right) \in \mathbf{F}_{M}^{s_{0}, \sigma}(I) \times \mathbf{V}_{+, m}^{s_{0}+1 / 2, \sigma}(I)$. In view of the existence of right and left limits of elements of $V^{2}$, this second fact is, roughly speaking, simply a restatement of Theorem 1. We give a more detailed description of this argument in Section 4.

We state the bilinear estimates that we exploit in the following.

THEOREM 2. Let $s_{0}, \sigma \geqslant 0$ and $M, m>0$ satisfy either (3) or (2). There exist $1<a<2, b>0$, and $C>0$ such that if $I \subset \mathbb{R}$ is a left-closed interval, $t_{0} \in I$, and $\phi_{+} \in \mathbf{V}_{m,+}^{s_{0}+1 / 2, \sigma}(I), \psi, \varphi \in \mathbf{F}_{M}^{s_{0}, \sigma}(I)$, then we have the bounds

$$
\begin{aligned}
\left\|\mathcal{I}_{t_{0}}^{M}\left(\operatorname{Re}\left(\phi_{+}\right) \gamma^{0} \psi\right)\right\|_{\mathbf{V}_{M}^{s_{0}, \sigma}(I)} \leqslant & C\left(\left\|\phi_{+}\right\|_{\mathbf{D}_{\sigma}^{0}(I)}\|\psi\|_{\mathbf{D}_{\sigma}^{-1 / 2}(I)}\right)^{\theta} \\
& \cdot\left(\left\|\phi_{+}\right\|_{\mathbf{V}_{+, m}^{s_{0}+1 / 2, \sigma}(I)}\|\psi\|_{\mathbf{F}_{M}^{s_{0}, \sigma}(I)}\right)^{1-\theta},
\end{aligned}
$$




$$
\begin{aligned}
\left\|\mathcal{I}_{t_{0}}^{M}\left(\operatorname{Re}\left(\phi_{+}\right) \gamma^{0} \psi\right)\right\|_{\mathbf{Y}_{M}^{s_{0}, \sigma}(I)} \leqslant & C\left(\left\|\phi_{+}\right\|_{\mathbf{D}_{\sigma}^{0}(I)}\|\psi\|_{\mathbf{D}_{\sigma}^{-1 / 2}(I)}\right)^{\theta} \\
& \cdot\left(\left\|\phi_{+}\right\|_{\mathbf{V}_{+, m}^{s_{0}+1 / 2, \sigma}(I)}\|\psi\|_{\mathbf{V}_{M}^{s_{0}, \sigma}(I)}\right)^{1-\theta},
\end{aligned}
$$

and

$$
\begin{aligned}
\left\|\mathcal{I}_{t_{0}}^{m,+}\left(\langle\nabla\rangle_{m}^{-1}(\bar{\psi} \varphi)\right)\right\|_{\mathbf{V}_{+, m}^{s_{0}+1 / 2, \sigma}(I)} \leqslant & C\left(\|\psi\|_{\mathbf{D}_{\sigma}^{-1 / 2}(I)}\|\varphi\|_{\mathbf{D}_{\sigma}^{-1 / 2}(I)}\right)^{\theta} \\
& \cdot\left(\|\psi\|_{\mathbf{F}_{M}^{s_{0}, \sigma}(I)}\|\varphi\|_{\mathbf{F}_{M}^{s_{0}, \sigma}(I)}\right)^{1-\theta} .
\end{aligned}
$$

Moreover, for any $s \geqslant s_{0}$, we have the fractional Leibniz type bounds

$$
\begin{aligned}
& \left\|\mathcal{I}_{t_{0}}^{M}\left(\operatorname{Re}\left(\phi_{+}\right) \gamma^{0} \psi\right)\right\|_{\mathbf{F}_{M}^{s, \sigma}(I)} \\
& \quad \leqslant 2^{s} C\left(\left\|\phi_{+}\right\|_{\mathbf{D}_{\sigma}^{0}(I)}^{\theta}\left\|\phi_{+}\right\|_{\mathbf{V}_{+, m}^{s_{0}+1 / 2, \sigma}(I)}^{1-\theta}\|\psi\|_{\mathbf{F}_{M}^{s, \sigma}(I)}+\left\|\phi_{+}\right\|_{\mathbf{V}_{+, m}^{s+1 / 2, \sigma}(I)}\|\psi\|_{\mathbf{D}_{\sigma}^{-1 / 2}(I)}^{\theta}\|\psi\|_{\mathbf{F}_{M}^{s, o}(I)}^{1-\theta}\right)
\end{aligned}
$$

and

$$
\begin{aligned}
& \left\|\mathcal{I}_{t_{0}}^{m,+}\left(\langle\nabla\rangle_{m}^{-1}(\bar{\psi} \varphi)\right)\right\|_{\mathbf{V}_{+, m}^{s+1 / 2, \sigma}(I)} \\
& \quad \leqslant 2^{s} C\left(\|\psi\|_{\mathbf{D}_{\sigma}^{-1 / 2}(I)}^{\theta}\|\psi\|_{\mathbf{F}_{M}^{s_{0}, \sigma}(I)}^{1-\theta}\|\varphi\|_{\mathbf{F}_{M}^{s, \sigma}(I)}+\|\psi\|_{\mathbf{F}_{M}^{s, \sigma}(I)}\|\varphi\|_{\mathbf{D}_{\sigma}^{-1 / 2}(I)}^{\theta}\|\varphi\|_{\mathbf{F}_{M}^{s_{0}, \sigma}(I)}^{1-\theta}\right) .
\end{aligned}
$$

Proof. First, suppose that $I=\mathbb{R}$. By rescaling, we may assume $m=1$. The bounds are immediate consequences of Theorem 7 (in case (2)) and Theorem 9 (in case (3)), because of $2 \operatorname{Re}\left(\phi_{+}\right)=\phi_{+}+\phi_{+}^{*}$, as well as the fact that for any $s^{\prime}$ and $m_{1} \geqslant 0$, we have $\|\cdot\|_{\mathbf{D}_{\sigma}^{s^{\prime}}} \lesssim\|\cdot\|_{\mathbf{V}_{ \pm, m_{1}}^{s^{\prime}+1 / 2, \sigma}}$.

Finally, we remark that Lemma 1 implies the claims for arbitrary left-closed intervals $I$ with $t_{0} \in I$, since we have

$$
\mathbb{1}_{I} \mathcal{I}_{t_{0}}^{M}\left(\operatorname{Re}\left(\phi_{+}\right) \gamma^{0} \psi\right)=\mathbb{1}_{I} \mathcal{I}_{t_{0}}^{M}\left(\operatorname{Re}\left(\mathbb{1}_{I} \phi_{+}\right) \gamma^{0} \mathbb{1}_{I} \psi\right)
$$

and similarly for $\mathcal{I}_{t_{0}}^{m,+}\left(\langle\nabla\rangle_{m}^{-1}(\bar{\psi} \varphi)\right)$.

Note that we have elected to separate the statement of the bounds in $\mathbf{F}_{M}^{s_{0}, \sigma}$ for spinor components into a $\mathbf{V}_{M}^{s_{0}, \sigma}$ bound, and a $\mathbf{Y}_{M}^{s_{0}, \sigma}$ bound. The motivation is that although the bound in $\mathbf{V}_{M}^{s_{0}, \sigma}$ requires $\psi \in \mathbf{F}_{M}^{s_{0}, \sigma}$, the control of the $\mathbf{Y}_{M}^{s_{0}, \sigma}$ norm only requires $\psi \in \mathbf{V}_{M}^{s_{0}, \sigma}$. Consequently, if required, by using a two-step iteration argument, it is possible to develop a local theory using just the $V^{2}$ type norms, without explicitly using the $\mathbf{Y}_{M}^{s_{0}, \sigma}$ spaces. Another way of stating this, is that for solutions to the DKG system, if $\psi \in \mathbf{V}_{M}^{s_{0}, \sigma}$, then we immediately have $\psi \in \mathbf{F}_{M}^{s_{0}, \sigma}$.

We now give a precise version of the local well-posedness (and small data global well-posedness) theory that follows from the bilinear estimates in Theorem 2. 
THEOREM 3 (Local well-posedness). Let $s_{0}, \sigma \geqslant 0$ and $M, m>0$ satisfy either (3) or (2). There exist $\theta \in(0,1)$ and $C>1$, such that if

$$
A, B>0, \quad 0<\alpha \leqslant\left(C A^{1-\theta}\right)^{-1 / \theta}, \quad 0<\beta \leqslant\left(C B^{1-\theta}\right)^{-1 / \theta},
$$

and $I \subset \mathbb{R}$ is a left-closed interval, then for any initial time $t_{0} \in I$, and any data $\left(\psi_{0}, \phi_{0}\right) \in H_{\sigma}^{s_{0}} \times H_{\sigma}^{s_{0}+1 / 2}$ satisfying

$$
\left\|\psi_{0}\right\|_{H_{\sigma}^{s_{0}}}<A, \quad\left\|\mathcal{U}_{M}\left(t-t_{0}\right) \psi_{0}\right\|_{\mathbf{D}_{\sigma}^{-1 / 2}(I)}<\alpha
$$

and

$$
\left\|\phi_{0}\right\|_{H_{\sigma}^{s_{0}+1 / 2}}<B, \quad\left\|\mathcal{U}_{m}^{+}\left(t-t_{0}\right) \phi_{0}\right\|_{\mathbf{D}_{\sigma}^{0}(I)}<\beta
$$

there exists a unique $H_{\sigma}^{s_{0}}$-strong solution $\left(\psi, \phi_{+}\right)$of (16) on I with ( $\psi$, $\left.\phi_{+}\right)\left(t_{0}\right)=\left(\psi_{0}, \phi_{0}\right)$. Moreover, the data-to-solution map is Lipschitz-continuous into $\mathbf{F}_{M}^{s_{0}, \sigma}(I) \times \mathbf{V}_{+, m}^{s_{0}, \sigma}(I)$ and we have the bounds

$$
\begin{aligned}
\left\|\psi-\mathcal{U}_{M}\left(t-t_{0}\right) \psi_{0}\right\|_{\mathbf{F}_{M}^{s_{0}, \sigma}(I)} & \leqslant C \alpha^{\theta} A^{1-\theta}\left(\alpha^{\theta} A^{1-\theta}+\beta^{\theta} B^{1-\theta}\right) \\
\left\|\phi_{+}-\mathcal{U}_{m}^{+}\left(t-t_{0}\right) \phi_{0}\right\|_{\mathbf{V}_{+, m}^{s_{0}+1 / 2, \sigma}} & \leqslant C\left(\alpha^{\theta} A^{1-\theta}\right)^{2} .
\end{aligned}
$$

Finally, if we have additional regularity $\left(\psi_{0}, \phi_{0}\right) \in H_{\sigma}^{s} \times H_{\sigma}^{s+1 / 2}$ for some $s>s_{0}$, then $\left(\psi, \phi_{+}\right) \in F_{M}^{s, \sigma}(I) \times \mathbf{V}_{+, m}^{s, \sigma}(I)$ is also an $H_{\sigma}^{s}$-strong solution.

Proof. Let $\epsilon_{0}=\alpha^{\theta} A^{1-\theta}+\beta^{\theta} B^{1-\theta}$ and $\epsilon_{s}=\left\|\psi_{0}\right\|_{H_{\sigma}^{s}}+\left\|\phi_{0}\right\|_{H_{\sigma}^{s+1 / 2}}$. For ease of notation, we take

$$
\psi_{L}=\mathcal{U}_{M}\left(t-t_{0}\right) \psi_{0}, \quad \phi_{L}=\mathcal{U}_{m}^{+}\left(t-t_{0}\right) \phi_{0}, \quad \psi_{N}=\psi-\psi_{L}, \quad \phi_{N}=\phi-\phi_{L} .
$$

Define the set $\mathcal{X}_{s} \subset \mathbf{F}_{M}^{s, \sigma}(I) \times \mathbf{V}_{+, m}^{s, \sigma}(I)$ as the collection of all $(\psi, \phi) \in \mathbf{F}_{M}^{s, \sigma}(I) \times$ $\mathbf{V}_{+, m}^{s, \sigma}(I)$ such that

$$
\left\|\psi_{N}\right\|_{\mathbf{F}_{M}^{s_{0}, \sigma}(I)}+\left\|\phi_{N}\right\|_{\mathbf{V}_{+, m}^{s_{0}, \sigma}(I)} \leqslant \epsilon_{0}, \quad\left\|\psi_{N}\right\|_{\mathbf{F}_{M}^{s, \sigma}(I)}+\left\|\phi_{N}\right\|_{\mathbf{V}_{M}^{s, \sigma}(I)} \leqslant \epsilon_{s} .
$$

Our goal is to construct a fixed point of the map $\mathcal{T}=\left(\mathcal{T}_{1}, \mathcal{T}_{2}\right): \mathcal{X}_{s} \rightarrow \mathcal{X}_{s}$ defined as

$$
\mathcal{T}_{1}(\psi, \phi)=\psi_{L}+\mathcal{I}_{t_{0}}^{M}\left[\operatorname{Re}(\phi) \gamma^{0} \psi\right], \quad \mathcal{T}_{2}(\psi, \phi)=\phi_{L}+\mathcal{I}_{t_{0}}^{+, m}\left[\langle\nabla\rangle_{m}^{-1}(\bar{\psi} \psi)\right] .
$$

To this end, if $(\psi, \phi) \in \mathcal{X}_{s}$, then after decomposing the product $\phi \psi=\phi_{L} \psi_{L}+$ $\phi_{N} \psi_{L}+\phi_{L} \psi_{N}+\psi_{N} \phi_{N}$, an application of Theorem 2 together with bounds (12) and (13) implies there exists $C^{*}>0$ such that

$$
\begin{aligned}
\left\|\mathcal{I}_{t_{0}}^{M}\left(\operatorname{Re}(\phi) \gamma^{0} \psi\right)\right\|_{\mathbf{F}_{M}^{s_{0}, \sigma}(I)} & \leqslant C^{*}\left(\beta^{\theta} B^{1-\theta}+\epsilon_{0}\right) \epsilon_{0} \\
\left\|\mathcal{I}_{t_{0}}^{M}\left(\operatorname{Re}(\phi) \gamma^{0} \psi\right)\right\|_{\mathbf{F}_{M}^{s, \sigma}(I)} & \leqslant C^{*}\left(\beta^{\theta} B^{1-\theta}+\epsilon_{0}\right) \epsilon_{s} .
\end{aligned}
$$


Similarly, decomposing $\bar{\psi} \psi=\bar{\psi}_{L} \psi_{L}+\bar{\psi}_{N} \psi_{L}+\bar{\psi}_{L} \psi_{N}+\bar{\psi}_{N} \psi_{N}$, we have $C^{*}>0$ such that

$$
\begin{aligned}
& \left\|\mathcal{I}_{t_{0}}^{+, m}\left(\langle\nabla\rangle_{m}^{-1} \bar{\psi} \psi\right)\right\|_{\mathbf{V}_{+, m}^{s_{0}, \sigma}(I)} \leqslant C^{*} \epsilon_{0}^{2} \\
& \left\|\mathcal{I}_{t_{0}}^{+, m}\left(\langle\nabla\rangle_{m}^{-1} \bar{\psi} \psi\right)\right\|_{\mathbf{V}_{+, m}^{s, \sigma}(I)} \leqslant C^{*} \epsilon_{0} \epsilon_{s} .
\end{aligned}
$$

To show that $\mathcal{T}$ is a contraction on $\mathcal{X}_{s}$, we observe that for $(\psi, \phi),\left(\psi^{\prime}, \phi^{\prime}\right) \in \mathcal{X}$ after decomposing the difference of the products as

$$
\phi \psi-\phi^{\prime} \psi^{\prime}=\left(\phi-\phi^{\prime}\right)\left(\psi_{L}+\psi_{N}\right)+\left(\phi_{L}+\phi_{N}^{\prime}\right)\left(\psi^{\prime}-\psi\right)
$$

another application of Theorem 2, (12), and (13) implies that there exists $C^{*}=$ $C^{*}(s)>0$ such that

$$
\begin{aligned}
& \left\|\mathcal{I}_{t_{0}}^{M}\left(\operatorname{Re}(\phi) \gamma^{0} \psi\right)-\mathcal{I}_{t_{0}}^{M}\left(\operatorname{Re}\left(\phi^{\prime}\right) \gamma^{0} \psi^{\prime}\right)\right\|_{\mathbf{F}_{M}^{s_{0}, \sigma}(I)} \\
& \quad \leqslant C^{*}\left(\beta^{\theta} B^{1-\theta}+\epsilon_{0}\right)\left(\left\|\psi-\psi^{\prime}\right\|_{\mathbf{F}_{M}^{s_{0}, \sigma}(I)}+\left\|\phi-\phi^{\prime}\right\|_{\mathbf{v}_{+, m}^{s_{0}, \sigma}(I)}\right)
\end{aligned}
$$

and

$$
\begin{aligned}
& \left\|\mathcal{I}_{t_{0}}^{M}\left(\operatorname{Re}(\phi) \gamma^{0} \psi\right)-\mathcal{I}_{t_{0}}^{M}\left(\operatorname{Re}\left(\phi^{\prime}\right) \gamma^{0} \psi^{\prime}\right)\right\|_{\mathbf{F}_{M}^{s, \sigma}(I)} \\
& \leqslant C^{*}\left(\beta^{\theta} B^{1-\theta}+\epsilon_{0}\right)\left(\left\|\psi-\psi^{\prime}\right\|_{\mathbf{F}_{M}^{s, \sigma}(I)}+\left\|\phi-\phi^{\prime}\right\|_{\mathbf{V}_{+, m}^{s, \sigma}(I)}\right) \\
& \quad+C^{*} \epsilon_{s}\left(\left\|\psi-\psi^{\prime}\right\|_{\mathbf{F}_{M}^{s, \sigma}(I)}+\left\|\phi-\phi^{\prime}\right\|_{\mathbf{v}_{+, m}^{s, \sigma}(I)}\right) .
\end{aligned}
$$

Similarly, we have $C^{*}=C^{*}(s)>0$ such that

$$
\begin{aligned}
& \left\|\mathcal{I}_{t_{0}}^{+, m}\left(\langle\nabla\rangle_{m}^{-1} \bar{\psi} \psi\right)-\mathcal{I}_{t_{0}}^{+, m}\left(\langle\nabla\rangle_{m}^{-1} \overline{\psi^{\prime}} \psi^{\prime}\right)\right\|_{\mathbf{V}_{+, m}^{s_{0}, \sigma}(I)} \\
& \quad \leqslant C^{*} \epsilon_{0}\left(\left\|\psi-\psi^{\prime}\right\|_{\mathbf{F}_{M}^{s_{0}, \sigma}(I)}+\left\|\phi-\phi^{\prime}\right\|_{\mathbf{V}_{+, m}^{s_{0}, \sigma}(I)}\right)
\end{aligned}
$$

and

$$
\begin{aligned}
\left\|\mathcal{I}_{t_{0}}^{+, m}\left(\langle\nabla\rangle_{m}^{-1} \bar{\psi} \psi\right)-\mathcal{I}_{t_{0}}^{+, m}\left(\langle\nabla\rangle_{m}^{-1} \overline{\psi^{\prime}} \psi^{\prime}\right)\right\|_{\mathbf{V}_{+, m}^{s, \sigma}(I)} & \\
\leqslant & C^{*} \epsilon_{0}\left(\left\|\psi-\psi^{\prime}\right\|_{\mathbf{F}_{M}^{s, \sigma}(I)}+\left\|\phi-\phi^{\prime}\right\|_{\mathbf{V}_{+, m}^{s, \sigma}(I)}\right) \\
& +C^{*} \epsilon_{s}\left(\left\|\psi-\psi^{\prime}\right\|_{\mathbf{F}_{M}^{s_{0}, \sigma}(I)}+\left\|\phi-\phi^{\prime}\right\|_{\mathbf{V}_{+, m}^{s, \sigma}(I)}\right) .
\end{aligned}
$$

Consequently, taking $C^{*}=C^{*}(s)>0$ to be the largest of the constants appearing in (17)-(22), we see that provided

$$
\beta^{\theta} B^{1-\theta}+\alpha^{\theta} A^{1-\theta} \leqslant\left(2 C^{*}\right)^{-1}
$$

we have $\mathcal{T}: \mathcal{X}_{s} \rightarrow \mathcal{X}_{s}$ and the difference bounds

$$
\begin{aligned}
& \left\|\mathcal{T}_{1}(\psi, \phi)-\mathcal{T}_{1}\left(\psi^{\prime}, \phi^{\prime}\right)\right\|_{\mathbf{F}_{M}^{s_{0}, \sigma}(I)}+\left\|\mathcal{T}_{2}(\psi, \phi)-\mathcal{T}_{2}\left(\psi^{\prime}, \phi^{\prime}\right)\right\|_{\mathbf{V}_{+, m}^{s_{0}, \sigma}(I)} \\
& \quad \leqslant \frac{1}{2}\left(\left\|\psi-\psi^{\prime}\right\|_{\mathbf{F}_{M}^{s_{0}, \sigma}(I)}+\left\|\phi-\phi^{\prime}\right\|_{\mathbf{V}_{+, m}^{s_{0}, \sigma}(I)}\right)
\end{aligned}
$$


and

$$
\begin{aligned}
& \left\|\mathcal{T}_{1}(\psi, \phi)-\mathcal{T}_{1}\left(\psi^{\prime}, \phi^{\prime}\right)\right\|_{\mathbf{F}_{M}^{s, \sigma}(I)}+\left\|\mathcal{T}_{2}(\psi, \phi)-\mathcal{T}_{2}\left(\psi^{\prime}, \phi^{\prime}\right)\right\|_{\mathbf{V}_{+, m}^{s, \sigma}(I)} \\
& \leqslant \frac{1}{2}\left(\left\|\psi-\psi^{\prime}\right\|_{\mathbf{F}_{M}^{s, \sigma}(I)}+\left\|\phi-\phi^{\prime}\right\|_{\mathbf{V}_{+, m}^{s, \sigma}(I)}\right) \\
& \quad+2 C^{*} \epsilon_{s}\left(\left\|\psi-\psi^{\prime}\right\|_{\mathbf{F}_{M}^{s, \sigma}(I)}+\left\|\phi-\phi^{\prime}\right\|_{\mathbf{V}_{+, m}^{s, \sigma}(I)}\right)
\end{aligned}
$$

Therefore, a standard argument implies that there exists a unique fixed point in $\mathcal{X}_{s}$, and that the resulting solution map depends continuously on the initial data.

Fix $C=\sup _{s_{0} \leqslant s \leqslant 10} 2 C^{*}(s)$ and suppose that $0<\alpha \leqslant\left(C A^{1-\theta}\right)^{-1 / \theta}$ and $0<\beta \leqslant\left(C B^{1-\theta}\right)^{-1 / \theta}$. Then running the above argument with $s=s_{0}$ shows that for data $\left(\psi_{0}, \phi_{0}\right) \in H_{\sigma}^{s_{0}} H_{\sigma}^{s_{0}+1 / 2}$ we get a unique solution $\left(\psi, \phi_{+}\right) \in \mathcal{X}_{s_{0}}$, which depends continuously on the data. Approximating the data with functions in $H_{\sigma}^{10} \times H_{\sigma}^{10+1 / 2}$ and applying the previous argument with $s=10$, we obtain a sequence of solutions in $\mathcal{X}_{10}$, which converge to $\left(\psi, \phi_{+}\right)$. In particular, $\left(\psi, \phi_{+}\right)$ is an $H_{\sigma}^{s_{0}}$-strong solution to (16). The claimed bounds on the norms of $\left(\psi, \phi_{+}\right)$ follow directly from the fact that $\left(\psi, \phi_{+}\right) \in \mathcal{X}$ together with (17) and (18). Finally, if in addition we have additional regularity $\left(\psi_{0}, \phi_{0}\right) \in H_{\sigma}^{s}$ with $s>s_{0}$, then running the fixed point argument as above gives a solution in $\mathbf{F}_{M}^{s, \sigma}\left(I^{\prime}\right) \times \mathbf{V}_{+, m}^{s, \sigma}\left(I^{\prime}\right)$ provided that the interval $I^{\prime} \subset I$ is chosen sufficiently small such that

$$
\left\|\psi_{L}\right\|_{\mathbf{D}_{\sigma}^{-1 / 2}\left(I^{\prime}\right)}^{\theta}\left\|\psi_{0}\right\|_{H_{\sigma}^{s_{0}}}^{1-\theta}+\left\|\phi_{L}\right\|_{\mathbf{D}_{\sigma}^{0}\left(I^{\prime}\right)}^{\theta}\left\|\phi_{0}\right\|_{H_{\sigma}^{s_{0}+1 / 2}}^{1-\theta} \leqslant\left(2 C^{*}(s)\right)^{-1} .
$$

Since we can cover the full interval $I$ by $\mathcal{O}_{s}(1)$ smaller intervals $I^{\prime}$, we deduce that $\left(\psi, \phi_{+}\right) \in \mathbf{F}_{M}^{s, \sigma}(I) \times \mathbf{V}_{M}^{s, \sigma}(I)$ as claimed.

Note that we may take $I=[0, \infty)$ in the previous theorem. In particular, if $\left(\psi_{0}, \phi_{0}\right) \in H_{\sigma}^{s_{0}} \times H_{\sigma}^{s_{0}+1 / 2}$ then provided $\left\|\mathcal{U}_{M}(t) \psi_{0}\right\|_{\mathbf{D}_{\sigma}^{-1 / 2}(I)}$ and $\left\|\mathcal{U}_{m}^{+}(t) \phi_{0}\right\|_{\mathbf{D}_{\sigma}^{0}(I)}$ are sufficiently small, we have global existence and scattering.

The next result we give implies that $H_{\sigma}^{s_{0}}$-strong solutions belong to $\mathbf{F}_{M}^{s_{0}, \sigma}(I) \times$ $\mathbf{V}_{+, m}^{s_{0}+1 / 2, \sigma}(I)$, provided that the $L_{t, x}^{4}$ type norm is sufficiently small relative to $\|(\psi$, $\left.\phi_{+}\right)\left(t_{0}\right) \|_{H_{\sigma}^{s_{0}} \times H_{\sigma}^{s_{0}+1 / 2}}$.

THEOREM 4. Let $s_{0}, \sigma \geqslant 0$ and $M, m>0$ satisfy either (3) or (2). There exist $C>1$ and $0<\theta<1$ such that, if $A>0$ and $I \subset \mathbb{R}$ is a left-closed interval, $t_{0} \in I$, and $\left(\psi, \phi_{+}\right)$is an $H_{\sigma}^{s_{0}}$-strong solution on I satisfying

$$
\left\|\psi\left(t_{0}\right)\right\|_{H_{\sigma}^{s_{0}}}+\left\|\phi_{+}\left(t_{0}\right)\right\|_{H_{\sigma}^{s_{0}+1 / 2}} \leqslant A
$$

and $\min \left\{\|\psi\|_{\mathbf{D}_{\sigma}^{-1 / 2}(I)},\left\|\phi_{+}\right\|_{\mathbf{D}_{\sigma}^{0}(I)}\right\} \leqslant\left(C(1+A)^{2-\theta} A^{1-\theta}\right)^{-1 / \theta}$,

then $\left(\psi, \phi_{+}\right) \in \mathbf{F}_{M}^{s_{0}, \sigma}(I) \times \mathbf{V}_{+, m}^{s_{0}+1 / 2, \sigma}(I)$ and we have the bound

$$
\|\psi\|_{\mathbf{F}_{M}^{s_{0}, \sigma}(I)}+\left\|\phi_{+}\right\|_{\mathbf{V}_{+, m}^{s_{0}+1 / 2, \sigma}(I)} \leqslant C A(1+A) .
$$


Proof. Let $C^{*}$ be the largest of the constants appearing in Lemma 1, Theorems 2 and 3 , and (12), (13). We first consider the case $I=\left[t_{0}, t_{1}\right)$ with $t_{1} \leqslant \infty$. Let $(\psi, \phi)$ be an $H_{\sigma}^{s_{0}}$-strong solution on $I$, and define

$$
\delta=\min \left\{\|\psi\|_{\mathbf{D}_{\sigma}^{-1 / 2}(I)},\left\|\phi_{+}\right\|_{\mathbf{D}_{\sigma}^{0}(I)}\right\}
$$

and

$$
\begin{aligned}
\mathcal{T}=\left\{t_{0}<T \leqslant t_{1} \mid\right. & \sup _{t_{0}<T^{\prime} \leqslant T}\|\psi\|_{\mathbf{F}_{M}^{s_{0}, \sigma}\left(\left[t_{0}, T^{\prime}\right)\right)} \leqslant 2 C^{*} A, \\
& \left.\sup _{t_{0}<T^{\prime} \leqslant T}\left\|\phi_{+}\right\|_{\mathbf{V}_{+, m}^{s_{0}+1 / 2, \sigma}\left(\left[t_{0}, T^{\prime}\right)\right)} \leqslant\left(2 C^{*}\right)^{3} A(1+A)\right\} .
\end{aligned}
$$

An application of the local well-posedness result in Theorem 3 implies that $T \in \mathcal{T}$ provided that $T-t_{0}$ is sufficiently small, in particular, $\mathcal{T}$ is nonempty. If we let $T_{\text {sup }}=\sup \mathcal{T}$, then our goal is to show that $T_{\text {sup }}=t_{1}$. Suppose that $T_{\text {sup }}<t_{1}$ and let $T_{n} \in \mathcal{T}$ be a sequence of times converging to $T_{\text {sup. }}$. The continuity of the solution $\left(\psi, \phi_{+}\right)$at $T_{\text {sup }}$, together with (13) and the definition of $\mathcal{T}$ implies that

$$
\begin{aligned}
\left\|\psi\left(T_{\text {sup }}\right)\right\|_{H_{\sigma}^{s_{0}}}+\left\|\phi_{+}\left(T_{\text {sup }}\right)\right\|_{H_{\sigma}^{s_{0}+1 / 2}} & \left.\leqslant C^{*} \sup _{t_{0}<T<T_{\text {sup }}}\|\psi\|_{\mathbf{F}_{M}^{s_{0}, \sigma}\left(\left[t_{0}, T\right)\right)}+\left\|\phi_{+}\right\|_{\mathbf{V}_{+, m}^{s_{0}+1 / 2, \sigma}\left(\left[t_{0}, T\right)\right)}\right) \\
& \leqslant\left(2 C^{*}\right)^{4} A(1+A) .
\end{aligned}
$$

Hence, again applying Theorem 3, there exist $n$ and $\epsilon_{0}>0$ such that for all $0<\epsilon<\epsilon_{0}$ we have on the interval $\left[T_{n}, T_{\text {sup }}+\epsilon\right)$ the bound

$$
\begin{aligned}
\|\psi\|_{\mathbf{F}_{M}^{s_{0}, \sigma}\left(\left[T_{n}, T_{\text {sup }}+\epsilon\right)\right)}+\|\phi\|_{\mathbf{V}_{+, m}^{s_{0}+1 / 2, \sigma}\left(\left[T_{n}, T_{\text {sup }}+\epsilon\right)\right)} & \leqslant 2 C^{*}\left(\left\|\psi\left(T_{\text {sup }}\right)\right\|_{H_{\sigma}^{s_{0}}}+\left\|\phi_{+}\left(T_{\text {sup }}\right)\right\|_{\left.H_{\sigma}^{s_{0}}\right)}\right. \\
& \leqslant\left(2 C^{*}\right)^{5} A(1+A) .
\end{aligned}
$$

We now exploit the smallness assumption on the $L_{t, x}^{4}$ norm. An application of (13), (15), and (14), together with Theorem 2 and the fact that $\left(\psi, \phi_{+}\right)$is an $H_{\sigma}^{s_{0}}$-strong solution on $\left[t_{0}, t_{1}\right)$ implies that

$$
\begin{aligned}
\|\psi\|_{\mathbf{F}_{M}^{s_{0}, \sigma}\left(\left[t_{0}, T_{\text {sup }}+\epsilon\right)\right)} \leqslant & C^{*}\left\|\psi\left(t_{0}\right)\right\|_{H_{\sigma}^{s_{0}}}+C^{*}\left(\|\psi\|_{\mathbf{D}_{\sigma}^{-1 / 2}\left(\left[t_{0}, T_{\text {sup }}+\epsilon\right)\right)}\left\|\phi_{+}\right\|_{\mathbf{D}_{\sigma}^{0}\left(\left[t_{0}, T_{\text {sup }}+\epsilon\right)\right)}\right)^{\theta} \\
& \cdot\left(\left(\|\psi\|_{\mathbf{F}_{M}^{s_{0}, \sigma}\left(\left[t_{0}, T_{n}\right)\right)}+\|\psi\|_{\mathbf{F}_{M}^{s_{0}, \sigma}\left(\left[T_{n}, T_{\text {sup }}+\epsilon\right)\right)}\right)\right. \\
& \left.\cdot\left(\left\|\phi_{+}\right\|_{\mathbf{V}_{+, m}^{s_{0}+1 / 2, \sigma}\left(\left[t_{0}, T_{n}\right)\right)}+\left\|\phi_{+}\right\|_{\mathbf{V}_{+, m}^{s_{0}+1 / 2, \sigma}\left(\left[T_{n}, T_{\text {sup }}+\epsilon\right)\right)}\right)\right)^{1-\theta} \\
\leqslant & C^{*} A+\delta^{\theta}\left(2 C^{*}\right)^{12}(1+A)^{2-\theta} A^{2-\theta} .
\end{aligned}
$$

Consequently, provided $\delta \leqslant\left[\left(2 C^{*}\right)^{12}(1+A)^{2-\theta} A^{1-\theta}\right]^{-1 / \theta}$, we see that

$$
\|\psi\|_{\mathbf{F}_{M}^{s_{0}, \sigma}\left(\left[t_{0}, T_{\text {sup }}+\epsilon\right)\right)} \leqslant 2 C^{*} A .
$$


To bound $\phi$, we simply observe that another application of Theorem 2 implies that

$$
\begin{aligned}
\left\|\phi_{+}\right\|_{\mathbf{V}_{+, m}^{s_{0}+1 / 2}\left(\left[t_{0}, T_{\text {sup }}+\epsilon\right)\right)} & \leqslant C^{*}\left\|\psi_{+}\left(t_{0}\right)\right\|_{H_{\sigma}^{s_{0}+1 / 2}}+C^{*}\|\psi\|_{\mathbf{F}_{M}^{s_{0}, \sigma}\left(\left[t_{0}, T_{\text {sup }}+\epsilon\right)\right)}^{2} \\
& \leqslant\left(2 C^{*}\right)^{3} A(1+A) .
\end{aligned}
$$

Therefore $T_{\text {sup }}+\epsilon \in \mathcal{T}$, which contradicts the assumption $T_{\text {sup }}<t_{1}$. Consequently, we must have $T_{\text {sup }}=t_{1}$ and hence $\psi \in \mathbf{F}_{M}^{s_{0}, \sigma}(I), \phi_{+} \in \mathbf{V}_{+, m}^{s_{0}+1 / 2}(I)$, and the claimed bounds hold. In the general case, when $t_{0}$ is not the left end point of $I$, we simply need to run the above argument for times smaller than $t_{0}$.

\section{Proof of Theorem 1 and Corollary 1}

Here we give the proof of Theorem 1 and Corollary 1.

4.1. Proof of Theorem 1. We first observe that since (1) and (16) are equivalent in the class of $H_{\sigma}^{s_{0}}$-strong solutions, and the Dirac-Klein-Gordon system is time reversible, it is enough to consider the forward-in-time problem for the reduced system (16). Thus let $\left(\psi, \phi_{+}\right):\left[t_{0}, t^{*}\right) \times \mathbb{R}^{3} \rightarrow \mathbb{C}^{4} \times \mathbb{C}$ be a forward maximal $H_{\sigma}^{s_{0}}$-solution to (16) such that

$$
\sup _{t \in\left[t_{0}, t^{*}\right)}\left(\|\psi(t)\|_{H_{\sigma}^{s_{0}}}+\left\|\phi_{+}(t)\right\|_{H_{\sigma}^{s_{0}+1 / 2}}\right) \leqslant A, \quad\|\psi\|_{\mathbf{D}_{\sigma}^{-1 / 2}\left(\left[t_{0}, t^{*}\right)\right)}<\infty .
$$

The finiteness of the dispersive norm $\|\cdot\|_{\mathbf{D}_{\sigma}^{-1 / 2}\left(\left[t_{0}, t^{*}\right)\right)}$, together with the dominated convergence theorem, implies that for every $\delta>0$ we can find an interval $I=\left[t_{1}\right.$, $\left.t^{*}\right)$ with $t_{1}<t^{*}$ such that

$$
\|\psi\|_{\mathbf{D}_{\sigma}^{-1 / 2}(I)} \leqslant \delta .
$$

In particular, choosing $\delta$ sufficiently small, depending only on $A$, an application of Theorem 4 implies that $\left(\psi, \phi_{+}\right) \in \mathbf{F}_{M}^{s_{0}, \sigma}(I) \times \mathbf{V}_{+, m}^{s_{0}+1 / 2, \sigma}(I)$. Therefore, by the existence of left limits in $V^{2}$, there exists $\left(\psi_{0}, \phi_{0}\right) \in H_{\sigma}^{s_{0}} \times H_{\sigma}^{s_{0}+1 / 2}$ such that

$$
\lim _{t \rightarrow t^{*}}\left(\left\|\psi(t)-\mathcal{U}_{M}(t) \psi_{0}\right\|_{H_{\sigma}^{s_{0}}}+\left\|\phi(t)-\mathcal{U}_{m}^{+}(t) \phi_{0}\right\|_{H_{\sigma}^{s_{0}+1 / 2}}\right)=0
$$

The local well-posedness theory in Theorem 3, together with the definition of maximal $H_{\sigma}^{s_{0}}$-solution, then implies that we must have $t^{*}=\infty$. Consequently, the solution $\left(\psi, \phi_{+}\right)$exists globally in time and scatters as $t \rightarrow \infty$. 
4.2. Proof of Corollary 1. In view of Theorem 1, and the fact that the spinor remains in $\mathcal{H}$ and that the wave component $\phi$ remains radial, it is enough to show that for $\psi_{0} \in \mathcal{H}, 1<p<\infty$, and $\sigma \geqslant 0$ we have the bound

$$
\sum_{N \in 2^{\mathbb{N}}} N^{\sigma}\left\|H_{N} \psi_{0}\right\|_{L_{x}^{p}} \leqslant\left\|\psi_{0}\right\|_{L_{x}^{p}}
$$

However, this follows directly from the definition of the angular frequency localization operators $H_{N}$, since the orthogonality of the spherical harmonics $y_{\ell, n}$ implies that for $\ell \geqslant 2$ and $j=1,2,3$ we have

$$
\left\langle 1, y_{l, n}\right\rangle_{L^{2}\left(\mathbb{S}^{2}\right)}=\left\langle\omega_{j}, y_{\ell, n}\right\rangle_{L^{2}\left(\mathbb{S}^{2}\right)}=0 .
$$

Therefore, $H_{N} \psi_{0}=0$ for $N \gg 1$ and hence (23) follows.

\section{Further notation and preliminary results}

In this section, we recall a number of results that will be used in the proof of the bilinear estimates in Theorem 2. The setup and notation follow closely our previous paper [6]; in particular, we refer the reader to [6] for further details and references.

We start by recalling the key fact that estimating a Duhamel term in $V_{ \pm, m}^{2}$ can be reduced to estimating a bilinear integral. More precisely, suppose that $F \in L_{t}^{\infty} L_{x}^{2}$, and

$$
\sup _{\left\|P_{\lambda} H_{N} v\right\|_{V_{ \pm, m}^{2}} \lesssim 1}\left|\int_{\mathbb{R}}\left\langle P_{\lambda} H_{N} v(t), F(t)\right\rangle_{L_{x}^{2}} d t\right|<\infty .
$$

If $u \in C\left(\mathbb{R}, L_{x}^{2}\right)$ satisfies $-i \partial_{t} u \pm\langle\nabla\rangle_{m} u=F$, then $P_{\lambda} H_{N} u \in V_{ \pm, m}^{2}$ and we have

$$
\left\|P_{\lambda} H_{N} u\right\|_{V_{ \pm, m}^{2}} \lesssim\left\|P_{\lambda} H_{N} u(0)\right\|_{L^{2}}+\sup _{\left\|P_{\lambda} H_{N} v\right\|_{V_{ \pm}^{2}, m} \lesssim 1} \int_{\mathbb{R}}\left\langle P_{\lambda} H_{N} v(t), F(t)\right\rangle_{L_{x}^{2}} d t .
$$

An analogous bound holds without the angular frequency multiplier $H_{N}$. See, for instance, [6, Lemma 7.3] for a proof of this inequality.

Let $\mathcal{Q}_{\mu}$ be a collection of cubes of diameter proportional to $\mu$ covering $\mathbb{R}^{3}$ with uniformly finite overlap, and let $\left(\rho_{q}\right)_{q \in \mathcal{Q}_{\mu}}$ be a subordinate partition of unity. For $q \in Q$ let $P_{q}=\rho_{q}(|-i \nabla|)$. Given $\alpha \leqslant 1$ and a collection $\mathcal{C}_{\alpha}$ of spherical caps $\kappa$ of diameter $\alpha$ with uniformly finite overlap, we let $\left(\rho_{\kappa}\right)_{\kappa \in \mathcal{C}_{\alpha}}$ be a smooth partition of unity subordinate to the conic sectors spanned by $\kappa$, and define the angular Fourier localization multipliers as $R_{\kappa}=\rho_{\kappa}(-i \nabla)$. In certain regimes, we need to use the fact that the modulation localization operators are uniformly disposable. 
LeMmA 2. Let $1 \leqslant q, r \leqslant \infty$, and $m>0$. For any $0<\alpha \leqslant 1, \lambda \in 2^{\mathbb{N}}, \kappa \in \mathcal{C}_{\alpha}$, $q \in \mathcal{Q}_{\alpha \lambda^{2}}$, and $d \in 2^{\mathbb{Z}}$ with $d \gtrsim \alpha^{2} \lambda$, we have

$$
\left\|C_{d}^{ \pm, m} R_{\kappa} P_{\lambda} P_{q} u\right\|_{L_{t}^{q} L_{x}^{r}}+\left\|C_{\leqslant d}^{ \pm, m} R_{\kappa} P_{\lambda} P_{q} u\right\|_{L_{t}^{q} L_{x}^{r}} \lesssim\left\|R_{\kappa} P_{\lambda} P_{q} u\right\|_{L_{t}^{q} L_{x}^{r}} .
$$

Here, if $\alpha \gtrsim \lambda^{-1}$, the operator $P_{q}$ can be dropped, and if $\lambda \approx 1$, the operator $R_{\kappa}$ can be dropped. Further, for every $d \in 2^{\mathbb{Z}}$

$$
\left\|C_{d}^{ \pm, m} u\right\|_{V_{ \pm, m}^{2}}+\left\|C_{\leqslant d}^{ \pm, m} u\right\|_{V_{ \pm, m}^{2}} \lesssim\|u\|_{V_{ \pm, m}^{2}} .
$$

Proof. First, it is enough to consider $0<\alpha \leqslant 1, \lambda \in 2^{\mathbb{N}}, r_{0} \geqslant 0$ satisfying $\left\langle r_{0}\right\rangle \approx \lambda$ and $d \in 2^{\mathbb{Z}}$ with $d \gtrsim \alpha^{2} \lambda$, and any function $u$ satisfying

$$
\operatorname{supp}(\widehat{u(t)}) \subset\left\{\xi \in \mathbb{R}^{3}|||\xi|-r_{0} \mid \lesssim \alpha \lambda^{2},\langle\xi\rangle \approx \lambda, r_{0}^{1 / 2}\left(|\xi|-\xi_{1}\right)^{1 / 2} \lesssim \lambda \alpha\right\},
$$

and to prove the estimate

$$
\left\|C_{\leqslant d}^{ \pm, m} u\right\|_{L_{t}^{q} L_{x}^{r}} \lesssim\|u\|_{L_{t}^{q} L_{x}^{r}} .
$$

The support assumptions imply that we can write

$$
C_{\leqslant d}^{ \pm, m} u=\omega * u
$$

for $\widetilde{\omega}$ a smooth bump function adapted to the set

$$
\left\{(\tau, \xi) \in \mathbb{R}^{1+3}|| \tau \pm\langle\xi\rangle|\lesssim d,||\xi|-r_{0} \mid \lesssim \alpha \lambda^{2},\langle\xi\rangle \approx \lambda, r_{0}^{1 / 2}\left(|\xi|-\xi_{1}\right)^{1 / 2} \lesssim \lambda \alpha\right\}
$$

Multiple integration by parts yields

$$
|\omega(t, x)| \lesssim_{N} d \beta \lambda(\alpha \lambda)^{2}\left(1+d|t|+\beta \lambda\left|x_{1} \pm t \frac{r_{0}}{\left\langle r_{0}\right\rangle}\right|+(\alpha \lambda)\left|\left(x_{2}, x_{3}\right)\right|\right)^{-N},
$$

where $\beta=\min \{1, \alpha \lambda\}$ and $N \in \mathbb{N}$. Clearly, this implies (25).

Second, from $C_{\leqslant d}^{ \pm, m}=\mathcal{U}_{m}^{ \pm} P_{\leqslant d} \mathcal{U}_{m}^{ \pm}(-\cdot)$ and

$$
\|\sigma * v\|_{V^{2}} \lesssim\|\sigma\|_{L^{1}}\|v\|_{V^{2}}
$$

the estimate (26) follows.

In a similar vein, for all $1 \leqslant p \leqslant \infty$, we note that

$$
\sup _{N \geqslant 1}\left\|H_{N}\right\|_{L^{p}\left(\mathbb{R}^{3}\right) \rightarrow L^{p}\left(\mathbb{R}^{3}\right)}<+\infty,
$$

and $H_{N}$ commutes with any radial Fourier multiplier such as $P_{\lambda}$. While $H_{N}$ also commutes with $C_{d}$, it does not commute with the cube and cap localization 
operators $R_{\kappa}$ and $P_{q}$. We also note an angular concentration bound [20, Lemma 5.2], which corresponds to [6, Lemma 8.5]. Let $2 \leqslant p<\infty$ and $0 \leqslant s<2 / p$. For all $\lambda, N \geqslant 1, \alpha \gtrsim \lambda^{-1}$, and $\kappa \in \mathcal{C}_{\alpha}$ we have

$$
\left\|R_{\kappa} P_{\lambda} H_{N} f\right\|_{L_{x}^{p}\left(\mathbb{R}^{3}\right)} \lesssim \alpha^{s} N^{s}\left\|P_{\lambda} H_{N} f\right\|_{L_{x}^{p}\left(\mathbb{R}^{3}\right)} .
$$

The proof of Theorem 2 requires carefully exploiting the structure of the product $\bar{\psi} \psi$. To this end, we recall a number of null form bounds that have been used frequently in the literature; see for instance, [6] and [1] for further details. We start by recalling that the multipliers $\Pi_{ \pm}$satisfy

$$
\left\|\left(\Pi_{ \pm_{1}}-\Pi_{ \pm_{1}}(\lambda \omega(\kappa))\right) R_{\kappa} P_{\lambda} f\right\|_{L_{x}^{r}} \lesssim \alpha\left\|R_{\kappa} P_{\lambda} u\right\|_{L_{x}^{r}}
$$

provided that $\lambda \geqslant 1, \alpha \gtrsim \lambda^{-1}$, and $\kappa \in \mathcal{C}_{\alpha}$. Similarly, in the Klein-Gordon regime we have

$$
\left\|\left(\Pi_{ \pm_{1}}-\Pi_{ \pm_{1}}\left(\xi_{0}\right)\right) R_{\kappa} P_{q} P_{\lambda} f\right\|_{L_{x}^{r}} \lesssim \alpha\left\|R_{\kappa} P_{q} P_{\lambda} u\right\|_{L_{x}^{r}},
$$

provided $\lambda \geqslant 1,0<\alpha \lesssim \lambda^{-1}, \kappa \in \mathcal{C}_{\alpha}$, and $q \in Q_{\lambda^{2} \alpha}$ with centre $\xi_{0}$. Consequently, the identity

$$
\begin{aligned}
{\left[\Pi_{ \pm_{1}} f\right]^{\dagger} \gamma^{0} \Pi_{ \pm_{2}} g=} & {\left[\left(\Pi_{ \pm_{1}}-\Pi_{ \pm_{1}}(x)\right) f\right]^{\dagger} \gamma^{0} \Pi_{ \pm_{2}} g } \\
& +\left[\Pi_{ \pm_{1}}(x) f\right]^{\dagger} \gamma^{0}\left(\Pi_{ \pm_{2}}-\Pi_{ \pm_{2}}(y)\right) g+f^{\dagger} \Pi_{ \pm_{1}}(x) \gamma^{0} \Pi_{ \pm_{2}}(y) g,
\end{aligned}
$$

together with the pointwise null form type bound

$$
\left|\Pi_{ \pm_{1}}(x) \gamma^{0} \Pi_{ \pm_{2}}(y)\right| \lesssim \theta\left( \pm_{1} x, \pm_{2} y\right)+\frac{\left| \pm_{1}\right| x\left| \pm_{2}\right| y||}{\langle x\rangle\langle y\rangle}
$$

then immediately implies, for instance, that

$$
\left\|\overline{\Pi_{ \pm_{1}} R_{\kappa} \psi_{\lambda_{1}}} \Pi_{ \pm_{2}} R_{\kappa^{\prime}} \varphi_{\lambda_{2}}\right\|_{L_{x}^{r}} \lesssim \alpha\|\psi\|_{L_{x}^{a}}\|\varphi\|_{L_{x}^{b}},
$$

where $1 / r=1 / a+1 / b, 1<r, a, b<\infty$, and the caps $\kappa, \kappa^{\prime} \in \mathcal{C}_{\alpha}$ satisfy $\mid \pm_{1} \kappa-$ $\pm_{2} \kappa^{\prime} \mid \lesssim \alpha$, with $\alpha \gtrsim\left(\min \left\{\lambda_{1}, \lambda_{2}\right\}\right)^{-1}$. Similarly, if we have the $V^{2}$ bound

$$
\left\|\psi^{\dagger} \varphi\right\|_{L_{x}^{r}} \leqslant \mathbf{C}\|\psi\|_{V_{ \pm_{1}, M}^{2}}\|\varphi\|_{V_{ \pm_{2}, M}^{2}}
$$

under some conditions on supp $\widehat{\psi}$ and supp $\widehat{\varphi}$, then we also have, under the same conditions on supp $\widehat{\psi}$ and supp $\widehat{\varphi}$, the null form bound

$$
\left\|\overline{\Pi_{ \pm_{1}} R_{\kappa} \psi} \Pi_{ \pm_{2}} R_{\kappa^{\prime}} \varphi\right\|_{L_{x}^{r}} \lesssim \alpha \mathbf{C}\|\psi\|_{V_{ \pm_{1}, M}^{2}}\|\varphi\|_{V_{ \pm_{2}, M}^{2}} .
$$

These estimates are used frequently in the proof of Theorem 2.

Next, we recall the Strichartz estimates for the wave equation, which corresponds to [6, Lemma 8.2] and relies on [1, Lemma 3.1] and [20, Appendix]. 
Lemma 3 (Wave Strichartz). Let $m \geqslant 0$ and $2<q \leqslant \infty$. If $0<\mu \leqslant \lambda, N \geqslant 1$, and $1 / r=\frac{1}{2}-1 / q$ then for every $q \in Q_{\mu}$ we have

$$
\left\|e^{\mp i t\langle\nabla\rangle_{m}} P_{q} P_{\lambda} f\right\|_{L_{t}^{q} L_{x}^{r}} \lesssim \mu^{1 / 2-1 / r} \lambda^{1 / 2-1 / r}\left\|P_{q} P_{\lambda} f\right\|_{L_{x}^{2}} .
$$

Moreover, if $1 / q+2 / r<1$ and $\epsilon>0$, we have

$$
\left\|e^{\mp i t\langle\nabla\rangle_{m}} P_{\lambda} H_{N} f\right\|_{L_{t}^{q} L_{x}^{r}} \lesssim \lambda^{3(1 / 2-1 / r)-1 / q} N^{1 / 2+\epsilon}\left\|P_{\lambda} H_{N} f\right\|_{L_{x}^{2}} .
$$

We also require Strichartz estimates in the Klein-Gordon regime. The first bound can be found in, for instance, [17, Lemma 3], while the second is a special case of [7, Theorem 1.1] and corresponds to [6, Lemma 8.3].

LEMmA 4 (Klein-Gordon Strichartz). Let $m>0$. Then for $\frac{1}{4} \leqslant 1 / r \leqslant \frac{3}{10}$ we have

$$
\left\|e^{\mp i t\langle\nabla\rangle_{m}} P_{\lambda} f\right\|_{L_{t, x}^{r}} \lesssim \lambda^{1 / 2}\left\|P_{\lambda} f\right\|_{L_{x}^{2}} .
$$

On the other hand, if $\frac{3}{10}<1 / r<\frac{5}{14}$ and $\epsilon>0$ we have

$$
\left\|e^{\mp i t\langle\nabla\rangle_{m}} P_{\lambda} H_{N} f\right\|_{L_{t, x}^{r}} \lesssim \lambda^{2-5 / r} N^{7(1 / r-3 / 10)+\epsilon}\left\|P_{\lambda} H_{N} f\right\|_{L_{x}^{2}} .
$$

Both Lemmas 3 and 4 have analogues in $V_{ \pm, m}^{2}$. This follows by decomposing into $U_{ \pm, m}^{2}$ atoms, and applying the estimate for free solutions; see for instance, the arguments used in [12, Proposition 2.19 and Corollary 2.21]. For instance, under the assumptions in Lemma 3, the first inequality in Lemma 3 implies that

$$
\left\|P_{q} P_{\lambda} u\right\|_{L_{t}^{q} L_{x}^{r}} \lesssim \mu^{1 / 2-1 / r} \lambda^{1 / 2-1 / r}\left\|P_{q} P_{\lambda} u\right\|_{V_{ \pm, m}^{2}} .
$$

Similarly, the remaining bounds in Lemmas 3 and 4 imply corresponding versions in $V_{ \pm, m}^{2}$.

We now turn to the bilinear estimates that we require in the proof of Theorem 2. To simplify the gain of the $L^{4}$ type norm in a particular high modulation interaction, we use the following 'cheap' bilinear $L_{t, x}^{2}$ estimate in $V^{2}$.

THEOREM 5. Let $\mu \ll \lambda_{1} \approx \lambda_{2}$. Then for any $0<\gamma \leqslant 1$ we have

$$
\begin{aligned}
& \left\|P_{\mu}\left(\overline{\Pi_{ \pm_{1}} \psi_{\lambda_{1}}} \Pi_{ \pm_{2}} \varphi_{\lambda_{2}}\right)\right\|_{L_{t, x}^{2}} \\
& \quad \lesssim \mu\left(\frac{\mu}{\lambda_{1}}\right)^{-2 \gamma}\left(\lambda_{1}^{-1 / 2}\left\|\psi_{\lambda_{1}}\right\|_{L_{t, x}^{4}} \lambda_{2}^{-1 / 2}\left\|\psi_{\lambda_{2}}\right\|_{L_{t, x}^{4}}\right)^{\gamma}\left(\left\|\psi_{\lambda_{1}}\right\|_{V_{ \pm_{1}, M}^{2}}\left\|\varphi_{\lambda_{2}}\right\|_{V_{ \pm_{2}, M}^{2}}\right)^{1-\gamma} .
\end{aligned}
$$

Proof. We consider separately the cases $\pm_{1}= \pm_{2}$ and $\pm_{1}=- \pm_{2}$. In the former case, the fact that the output frequencies are restricted to be of size $\mu$, implies that 
we can directly exploit the null structure together with the standard $L_{t, x}^{4}$ Strichartz bound to deduce that for every $\epsilon>0$ we have

$$
\begin{aligned}
&\left\|P_{\mu}\left(\overline{\Pi_{ \pm_{1}} \psi_{\lambda_{1}}} \Pi_{ \pm_{2}} \varphi_{\lambda_{2}}\right)\right\|_{L_{t, x}^{2}} \lesssim \frac{\mu}{\lambda_{1}} \sum_{\substack{q, q^{\prime} \in Q_{\mu} \\
\left|q-q^{\prime}\right| \approx \mu}}\left\|P_{q} \psi_{\lambda_{1}}\right\|_{L_{t, x}^{4}}\left\|P_{q^{\prime}} \varphi_{\lambda_{2}}\right\|_{L_{t, x}^{4}} \\
& \lesssim \mu\left(\frac{\mu}{\lambda_{1}}\right)^{1 / 2-\epsilon}\left\|\psi_{\lambda_{1}}\right\|_{V_{ \pm_{1}, M}^{2}}\left\|\varphi_{\lambda_{2}}\right\|_{V_{ \pm_{2}, M}^{2}} .
\end{aligned}
$$

On the other hand, in the $\pm_{1}=- \pm_{2}$ case, the frequency restriction implies that the free waves $\left(-i \partial_{t} \pm_{1}\langle\nabla\rangle_{M}\right) \psi=\left(-i \partial_{t} \pm_{2}\langle\nabla\rangle\right) \varphi=0$ are fully transverse. In particular, for free waves, we have the bilinear estimate

$$
\left\|P_{\mu}\left(\overline{\Pi_{ \pm_{1}} \psi_{\lambda_{1}}} \Pi_{ \pm_{2}} \varphi_{\lambda_{2}}\right)\right\|_{L_{t, x}^{2}} \lesssim \mu\left\|\psi_{\lambda_{1}}(0)\right\|_{L_{x}^{2}}\left\|\varphi_{\lambda_{2}}(0)\right\|_{L_{x}^{2}}
$$

see for instance [6, Lemma 2.6]. Arguing as in [12, Proposition 2.19 and Corollary 2.21], by interpolating this with the standard $L_{t, x}^{4}$ bound, we deduce that for every $\epsilon>0$ we have the $V_{ \pm, M}^{2}$ bound

$$
\left\|P_{\mu}\left[\left(\Pi_{ \pm_{1}} \psi_{\lambda_{1}}\right)^{\dagger} \gamma^{0} \Pi_{ \pm_{2}} \varphi_{\lambda_{2}}\right]\right\|_{L_{t, x}^{2}} \lesssim \mu\left(\frac{\mu}{\lambda_{1}}\right)^{-\epsilon}\left\|\psi_{\lambda_{1}}\right\|_{V_{ \pm_{1}, M}^{2}}\left\|\varphi_{\lambda_{2}}\right\|_{V_{ \pm_{2}, M}^{2}} .
$$

Thus in either of the \pm cases, by $L^{p}$ interpolation, we obtain

$$
\begin{aligned}
& \left\|P_{\mu}\left[\left(\Pi_{ \pm_{1}} \psi_{\lambda_{1}}\right)^{\dagger} \gamma^{0} \Pi_{ \pm_{2}} \varphi_{\lambda_{2}}\right]\right\|_{L_{t, x}^{2}} \\
& \quad \lesssim \mu\left(\frac{\mu}{\lambda_{1}}\right)^{-\epsilon(1-\gamma)-\gamma}\left(\lambda_{1}^{-1 / 2}\left\|\psi_{\lambda_{1}}\right\|_{L_{t, x}^{4}} \lambda_{2}^{-1 / 2}\left\|\psi_{\lambda_{2}}\right\|_{L_{t, x}^{4}}\right)^{\gamma}\left(\left\|\psi_{\lambda_{1}}\right\|_{V_{ \pm_{1}, M}^{2}}\left\|\varphi_{\lambda_{2}}\right\|_{V_{ \pm_{2}, M}^{2}}\right)^{1-\gamma} .
\end{aligned}
$$

Therefore, the result follows by choosing $\epsilon$ sufficiently small.

The main bilinear input in the critical case is the following bilinear restriction type bound from [4], which extends the corresponding bound in [6]. The key point in the following is that the estimate holds for functions in $V_{ \pm, m}^{2}$, in the full bilinear range. This is a highly nontrivial observation, which, in contrast to the linear and bilinear estimates mentioned above, does not follow from the same bounds for free homogeneous solutions. Instead it requires a more involved direct argument; see $[4,6]$ for further details.

THEOREM 6. Let $\epsilon>0,1 \leqslant q, r \leqslant 2,1 / q+2 / r<2$. For all $m_{1}, m_{2} \geqslant 0$, $0<\alpha \leqslant 1$, and $\xi_{0}, \eta_{0} \in \mathbb{R}^{3}$ such that $\left\langle\xi_{0}\right\rangle_{m_{1}} \approx \lambda_{1},\left\langle\eta_{0}\right\rangle_{m_{2}} \approx \lambda_{2}$, and

$$
\frac{\left|m_{2}\right| \xi_{0}\left|-m_{1}\right| \eta_{0}||}{\lambda_{1} \lambda_{2}}+\left(\frac{\left|\xi_{0}\right|\left|\eta_{0}\right| \mp \xi_{0} \cdot \eta_{0}}{\lambda_{1} \lambda_{2}}\right)^{1 / 2} \approx \alpha,
$$


and for all $u, v$ satisfying

$$
\begin{aligned}
& \operatorname{supp} \widehat{u} \subset\left\{|| \xi|-| \xi_{0}|| \ll \beta \lambda_{1},\left(|\xi|\left|\xi_{0}\right|-\xi \cdot \xi_{0}\right)^{1 / 2} \ll \alpha \lambda_{1}\right\}, \\
& \operatorname{supp} \widehat{v} \subset\left\{|| \xi|-| \eta_{0}|| \ll \beta \lambda_{2},\left(|\xi|\left|\eta_{0}\right|-\xi \cdot \eta_{0}\right)^{1 / 2} \ll \alpha \lambda_{2}\right\},
\end{aligned}
$$

then we have the bilinear estimate

$$
\|u v\|_{L_{t}^{q} L_{x}^{r}} \lesssim \alpha^{2-2 / r-2 / q} \beta^{1-1 / r} \lambda_{\min }^{3-3 / r-1 / q}\left(\frac{\lambda_{\max }}{\lambda_{\min }}\right)^{1 / q-1 / 2+\epsilon}\|u\|_{V_{ \pm_{1}, m_{1}}^{2}}\|v\|_{V_{ \pm_{2}, m_{2}}^{2}},
$$

where $\lambda_{\min }=\min \left\{\lambda_{1}, \lambda_{2}\right\}, \lambda_{\max }=\max \left\{\lambda_{1}, \lambda_{2}\right\}$, and $\beta=\left(m_{1} / \alpha \lambda_{1}+\right.$ $\left.m_{2} / \alpha \lambda_{2}+1\right)^{-1}$.

\section{Multilinear estimates in the subcritical case}

Our aim in this section is to establish the following result, which applies to the nonresonant regime. Here, after rescaling, we have $m=1$ and $M>\frac{1}{2}$ now.

THEOREM 7. Let $M>\frac{1}{2}$. If $s_{0}>0$ is sufficiently small, there exist $0<\theta<1$, $1<a<2$, and $C>1$, such that if $b=4\left(1 / a-\frac{1}{2}\right)$, for all $s \geqslant s_{0}>0$

$$
\begin{aligned}
& \left\|\Pi_{ \pm_{1}} \mathcal{I}_{M}^{ \pm_{1}}\left[\phi \gamma^{0} \Pi_{ \pm_{2}} \varphi\right]\right\|_{\mathbf{V}_{ \pm_{1}, M}^{s}} \\
& \lesssim \sup _{\mu, \lambda_{2} \geqslant 1}\left(\left\|\phi_{\mu}\right\|_{L_{t, x}^{4}} \lambda_{2}^{s-s_{0}-1 / 2}\left\|\varphi_{\lambda_{2}}\right\|_{L_{t, x}^{4}}\right)^{\theta}\left(\|\phi\|_{\mathbf{v}_{+, 1}^{1 / 2+s_{0}}}\|\varphi\|_{\mathbf{F}_{ \pm_{2}, M}^{s}}\right)^{1-\theta} \\
& \quad+\sup _{\mu, \lambda_{2} \geqslant 1}\left(\mu^{s-s_{0}}\left\|\phi_{\mu}\right\|_{L_{t, x}^{4}} \lambda_{2}^{-1 / 2}\left\|\varphi_{\lambda_{2}}\right\|_{L_{t, x}^{4}}\right)^{\theta}\left(\|\phi\|_{\mathbf{V}_{+, 1}^{1 / 2+s}}\|\varphi\|_{\mathbf{F}_{ \pm_{2}, M}^{s_{0}}}\right)^{1-\theta}
\end{aligned}
$$

and

$$
\begin{aligned}
& \left\|\Pi_{ \pm_{1}} \mathcal{I}_{M}^{ \pm_{1}}\left[\phi \gamma^{0} \Pi_{ \pm_{2}} \varphi\right]\right\|_{\mathbf{Y}_{ \pm_{1}, M}^{s}} \\
& \lesssim \sup _{\mu, \lambda_{2} \geqslant 1}\left(\left\|\phi_{\mu}\right\|_{L_{t, x}^{4}} \lambda_{2}^{s-s_{0}-1 / 2}\left\|\varphi_{\lambda_{2}}\right\|_{L_{t, x}^{4}}\right)^{\theta}\left(\|\phi\|_{\mathbf{V}_{+, 1}^{1 / 2+s_{0}}}\|\varphi\|_{\mathbf{V}_{ \pm_{2}, M}^{s}}\right)^{1-\theta} \\
& \quad+\sup _{\mu, \lambda_{2} \geqslant 1}\left(\mu^{s-s_{0}}\left\|\phi_{\mu}\right\|_{L_{t, x}^{4}} \lambda_{2}^{-1 / 2}\left\|\varphi_{\lambda_{2}}\right\|_{L_{t, x}^{4}}\right)^{\theta}\left(\|\phi\|_{\mathbf{V}_{+, 1}^{1 / 2+s}}\|\varphi\|_{\mathbf{V}_{ \pm_{2}, M}^{s_{0}}}\right)^{1-\theta}
\end{aligned}
$$

Similarly,

$$
\begin{aligned}
& \left\|\langle\nabla\rangle^{-1} \mathcal{I}_{m}^{+}\left[\left(\Pi_{ \pm_{1}} \psi\right)^{\dagger} \gamma^{0} \Pi_{ \pm_{2}} \varphi\right]\right\|_{\mathbf{V}_{+, 1}^{1 / 2+s}} \\
& \lesssim \sup _{\lambda_{1}, \lambda_{2} \geqslant 1}\left(\lambda_{1}^{-1 / 2}\left\|\psi_{\lambda_{1}}\right\|_{L_{t, x}^{4}} \lambda_{2}^{s-s_{0}-1 / 2}\left\|\varphi_{\lambda_{2}}\right\|_{L_{t, x}^{4}}\right)^{\theta}\left(\|\psi\|_{\mathbf{F}_{ \pm_{1}, M}^{s_{0}}}\|\varphi\|_{\mathbf{F}_{ \pm_{2}, M}^{s}}\right)^{1-\theta} \\
& \quad+\sup _{\lambda_{1}, \lambda_{2} \geqslant 1}\left(\lambda_{1}^{s-s_{0}-1 / 2}\left\|\psi_{\lambda_{1}}\right\|_{L_{t, x}^{4}} \lambda_{2}^{-1 / 2}\left\|\varphi_{\lambda_{2}}\right\|_{L_{t, x}^{4}}\right)^{\theta}\left(\|\psi\|_{\mathbf{F}_{ \pm_{1}, M}^{s}}\|\varphi\|_{\mathbf{F}_{ \pm_{2}, M}^{s_{0}}}\right)^{1-\theta}
\end{aligned}
$$

The proof, which we postpone to Section 6.2, relies on trilinear estimates. 
6.1. A subcritical trilinear estimate. Here, we consider frequency localized estimates and use the shorthand notation $f_{\lambda}=P_{\lambda} f$.

THEOREM 8. Let $M>\frac{1}{2}, 0<\varrho \ll 1, \frac{5}{3}<a<2$, and $0<b<\varrho / 4$. Let $\phi: \mathbb{R}^{1+3} \rightarrow \mathbb{C}$, and $\varphi, \psi: \mathbb{R}^{1+3} \rightarrow \mathbb{C}^{4}$ such that $\Pi_{ \pm_{1}} \psi=\psi$ and $\Pi_{ \pm_{2}} \varphi=\varphi$. Define

$$
\mathbf{A}=\left\|\phi_{\mu}\right\|_{L_{t, x}^{4}} \lambda_{1}^{-1 / 2}\left\|\psi_{\lambda_{1}}\right\|_{L_{t, x}^{4}} \lambda_{2}^{-1 / 2}\left\|\varphi_{\lambda_{2}}\right\|_{L_{t, x}^{4}} .
$$

There exists $\theta_{0} \in(0,1)$ such that

$$
\begin{aligned}
& \left|\int_{\mathbb{R}^{1+3}} \phi_{\mu} \overline{\psi_{\lambda_{1}}} \varphi_{\lambda_{2}} d x d t\right| \\
& \quad \lesssim \mu^{\varrho}\left(\frac{\mu}{\max \left\{\lambda_{1}, \lambda_{2}\right\}}\right)^{1 / 10} \mathbf{A}^{\theta_{0}}\left(\mu^{1 / 2}\left\|\phi_{\mu}\right\|_{V_{+, 1}^{2}}\left\|\psi_{\lambda_{1}}\right\|_{V_{ \pm_{1}, M}^{2}}\left\|\varphi_{\lambda_{2}}\right\|_{V_{ \pm_{2}, M}^{2}}\right)^{1-\theta_{0}} .
\end{aligned}
$$

If $\lambda_{1} \gg \lambda_{2}$, we can improve this to

$$
\begin{aligned}
& \left|\int_{\mathbb{R}^{1+3}} \phi_{\mu} \overline{\psi_{\lambda_{1}}} \varphi_{\lambda_{2}}-\sum_{d \lesssim \lambda_{2}} C_{\leqslant d} \phi_{\mu} \overline{\mathcal{C}_{\leqslant d}^{ \pm_{1}} \psi_{\lambda_{1}}} \mathcal{C}_{d}^{ \pm_{2}} \varphi_{\lambda_{2}} d x d t\right| \\
& \quad \lesssim \lambda_{2}^{\varrho}\left(\frac{\lambda_{2}}{\lambda_{1}}\right)^{1 / 10} \mathbf{A}^{\theta_{0}}\left(\mu^{1 / 2}\left\|\phi_{\mu}\right\|_{V_{+, 1}^{2}}\left\|\psi_{\lambda_{1}}\right\|_{V_{ \pm_{1}, M}^{2}}\left\|\varphi_{\lambda_{2}}\right\|_{V_{ \pm_{2}, M}^{2}}\right)^{1-\theta_{0}}
\end{aligned}
$$

and

$$
\begin{aligned}
& \left|\int_{\mathbb{R}^{1+3}} \sum_{d \lesssim \lambda_{2}} C_{\leqslant d} \phi_{\mu} \overline{\mathcal{C}_{\leqslant d}^{ \pm 1} \psi_{\lambda_{1}}} \mathcal{C}_{d}^{ \pm 2} \varphi_{\lambda_{2}} d x d t\right| \\
& \quad \lesssim \lambda_{2}^{\varrho}\left(\frac{\lambda_{2}}{\lambda_{1}}\right)^{(1 / 4)(1 / a-1 / 2)} \mathbf{A}^{\theta_{0}}\left(\mu^{1 / 2}\left\|\phi_{\mu}\right\|_{V_{+, 1}^{2}}\left\|\psi_{\lambda_{1}}\right\|_{V_{ \pm_{1}, M}^{2}}\|\varphi\|_{Y_{\lambda_{2}}^{ \pm}, M}\right)^{1-\theta_{0}} .
\end{aligned}
$$

Similarly, if $\lambda_{1} \ll \lambda_{2}$, we have

$$
\begin{aligned}
& \left|\int_{\mathbb{R}^{1+3}} \phi_{\mu} \overline{\psi_{\lambda_{1}}} \varphi_{\lambda_{2}}-\sum_{d \leqslant \lambda_{1}} C_{\leqslant d} \phi_{\mu} \overline{\mathcal{C}_{d}^{ \pm_{1}} \psi_{\lambda_{1}}} \mathcal{C}_{\leqslant d}^{ \pm_{2}} \varphi_{\lambda_{2}} d x d t\right| \\
& \quad \lesssim \lambda_{1}^{\varrho}\left(\frac{\lambda_{1}}{\lambda_{2}}\right)^{1 / 10} \mathbf{A}^{\theta_{0}}\left(\mu^{1 / 2}\left\|\phi_{\mu}\right\|_{V_{+, 1}^{2}}\left\|\psi_{\lambda_{1}}\right\|_{V_{ \pm_{1}, M}^{2}}\left\|\varphi_{\lambda_{2}}\right\|_{V_{ \pm_{2}, M}^{2}}\right)^{1-\theta_{0}}
\end{aligned}
$$

and

$$
\begin{aligned}
& \left|\int_{\mathbb{R}^{1+3}} \sum_{d \lesssim \lambda_{1}} C_{\leqslant d} \phi_{\mu} \overline{\mathcal{C}_{d}^{ \pm_{1}} \psi_{\lambda_{1}}} \mathcal{C}_{\leqslant d}^{ \pm 2} \varphi_{\lambda_{2}} d x d t\right| \\
& \quad \lesssim \lambda_{1}^{\varrho}\left(\frac{\lambda_{1}}{\lambda_{2}}\right)^{(1 / 4)(1 / a-1 / 2)} \mathbf{A}^{\theta_{0}}\left(\mu^{1 / 2}\left\|\phi_{\mu}\right\|_{V_{+, 1}^{2}}\|\psi\|_{Y_{\lambda_{1}}^{ \pm_{1}, M}}\left\|\varphi_{\lambda_{2}}\right\|_{V_{ \pm_{2}, M}^{2}}\right)^{1-\theta_{0}}
\end{aligned}
$$


Proof. The first step is to decompose the trilinear product into the modulation localized terms

$$
\phi_{\mu}\left(\Pi_{ \pm_{1}} \psi_{\lambda_{1}}\right)^{\dagger} \gamma^{0} \Pi_{ \pm_{2}} \varphi_{\lambda_{2}}=\sum_{d} A_{0}+A_{1}+A_{2}
$$

where

$$
\begin{aligned}
& A_{0}=C_{d} \phi_{\mu}\left(\mathcal{C}_{\ll d}^{ \pm \psi_{1}} \psi_{\lambda_{1}}\right)^{\dagger} \gamma^{0} \mathcal{C}_{\ll d}^{ \pm_{2}} \varphi_{\lambda_{2}}, \\
& A_{1}=C_{\lesssim d} \phi_{\mu}\left(\mathcal{C}_{d}^{ \pm 1} \psi_{\lambda_{1}}\right)^{\dagger} \gamma^{0} \mathcal{C}_{\lesssim d}^{ \pm 2} \varphi_{\lambda_{2}}, \\
& A_{2}=C_{\lesssim d} \phi_{\mu}\left(\mathcal{C}_{{ }_{d}}^{ \pm 1} \psi_{\lambda_{1}}\right)^{\dagger} \gamma^{0} \mathcal{C}_{d}^{ \pm 2} \varphi_{\lambda_{2}} .
\end{aligned}
$$

We now consider separately the small modulation cases:

$$
\mu \lesssim \lambda_{1} \approx \lambda_{2} \text { and } d \lesssim \mu, \quad \mu \gg \min \left\{\lambda_{1}, \lambda_{2}\right\} \text { and } d \lesssim \min \left\{\lambda_{1}, \lambda_{2}\right\}
$$

and the high modulation cases:

$$
\mu \lesssim \lambda_{1} \approx \lambda_{2} \text { and } d \gg \mu, \quad \mu \gg \min \left\{\lambda_{1}, \lambda_{2}\right\} \text { and } d \gg \min \left\{\lambda_{1}, \lambda_{2}\right\} .
$$

Due to the $L_{t, x}^{4}$ Strichartz inequality we have the obvious bound

$$
\mathbf{A} \lesssim \mu^{1 / 2}\left\|\phi_{\mu}\right\|_{V_{+, 1}^{2}}\left\|\psi_{\lambda_{1}}\right\|_{V_{ \pm_{1}, M}^{2}}\left\|\varphi_{\lambda_{2}}\right\|_{V_{ \pm_{2}, M}^{2}} .
$$

Therefore, if we have the bounds (37), (38), and (40) for some $\theta_{0}$, we can always replace $\theta_{0}$ with a smaller factor. In particular, in each of the various cases considered below, it is enough to get some (potentially very small) power of $\mathbf{A}$; the factor $\theta_{0}$ can then be taken to the minimum of the powers obtained. In the remaining estimates involving the $\|\cdot\|_{Y_{\lambda_{j}}^{ \pm_{j}, M}}$-norms, as this norm does not give control of the $L_{t, x}^{4}$ norm, we directly verify the fact that it is possible to make the exponent of A smaller if needed.

Before we start the case-by-case analysis, we recall some facts from the modulation analysis for the Dirac-Klein-Gordon system [1, 6, 9]. As in [6, Section 8B], we define the modulation function

$$
\mathfrak{M}_{ \pm_{1}, \pm_{2}}(\xi, \eta)=\left|\langle\xi-\eta\rangle \mp_{1}\langle\xi\rangle \pm_{2}\langle\eta\rangle\right|,
$$

where we take $\xi \in \operatorname{supp} \widehat{\psi}, \eta \in \operatorname{supp} \widehat{\varphi}$, and $\xi-\eta \in \operatorname{supp} \widehat{\phi}$. A computation shows that, for any $j=0,1,2$,

$$
\int_{\mathbb{R}^{1+3}} A_{j} d x d t \neq 0 \text { implies } \mathfrak{M}_{ \pm_{1}, \pm_{2}}(\xi, \eta) \lesssim d \text { within the domain of integration. }
$$

Furthermore, since $m=1$ and $M>\frac{1}{2}$ we are in the nonresonant case where

$$
\mathfrak{M}_{ \pm_{1}, \pm_{2}} \gtrsim\left(\min \left\{\mu, \lambda_{1}, \lambda_{2}\right\}\right)^{-1} \text {. }
$$

In particular, in the case-by-case analysis below, we may assume that $d \gtrsim$ $\left(\min \left\{\mu, \lambda_{1}, \lambda_{2}\right\}\right)^{-1}$. 
Case 1: $\mu \lesssim \lambda_{1} \approx \lambda_{2}$ and $d \lesssim \mu$. We first observe that the sum over the modulation is restricted to the region $\mu^{-1} \lesssim d \lesssim \mu$. Moreover, the resonance identities in [6, Lemma 8.7] together with (42) imply that

$$
d \gtrsim \mathfrak{M}_{ \pm_{1}, \pm_{2}}(\xi, \eta) \gtrsim \frac{\lambda_{1}^{2}}{\mu} \theta^{2}\left( \pm_{1} \xi, \pm_{2} \eta\right)+\mu \theta^{2}\left(\xi-\eta, \pm_{1} \xi\right)+\mu \theta^{2}\left(\xi-\eta, \pm_{2} \eta\right)
$$

Consequently, the angle between the Fourier supports of $\psi$ and $\varphi$ must be of size $\alpha=\left(d \mu / \lambda_{1}^{2}\right)^{1 / 2}$. In particular, decomposing $\psi$ and $\varphi$ into caps of size $\alpha$ and cubes of size $\mu$, applying Hölder's inequality, and using the null form bound (32) we deduce that for every $0<\delta<1$ :

$$
\begin{aligned}
& \left|\int_{\mathbb{R}^{1+3}} A_{0} d x d t\right| \\
& \quad \lesssim \alpha \sum_{\substack{q, q^{\prime} \in \mathcal{Q}_{\mu} \\
\left|q-q^{\prime}\right| \approx \mu}} \sum_{\substack{\kappa, \kappa^{\prime} \in \mathcal{C}_{\alpha} \\
\left| \pm_{1}- \pm_{2} \kappa^{\prime}\right| \approx \alpha}}\left\|C_{d} \phi_{\mu}\right\|_{L_{t, x}^{2}}\left\|P_{q} R_{\kappa} \psi_{\lambda_{1}}\right\|_{L_{t, x}^{4}}\left\|P_{q^{\prime}} R_{\kappa^{\prime}} \varphi_{\lambda_{2}}\right\|_{L_{t, x}^{4}} \\
& \quad \lesssim\left(\frac{d}{\mu}\right)^{-2 \delta}\left(\frac{\mu}{\lambda_{1}}\right)^{1 / 2-8 \delta} \mu^{1 / 2}\left\|\phi_{\mu}\right\|_{V_{+, 1}^{2}}\left(\lambda_{1}^{-1 / 2} \lambda_{2}^{-1 / 2}\left\|\psi_{\lambda_{1}}\right\|_{L_{t, x}^{4}}\left\|\varphi_{\lambda_{2}}\right\|_{L_{t, x}^{4}}\right)^{\delta} \\
& \quad \cdot\left(\left\|\psi_{\lambda_{1}}\right\|_{V_{ \pm_{1}, M}^{2}}\left\|\varphi_{\lambda_{2}}\right\|_{V_{ \pm_{2}, M}^{2}}\right)^{1-\delta},
\end{aligned}
$$

where we absorbed the square over caps and cubes using [6, Lemma 8.6], which gives, in particular

$$
\begin{aligned}
& \left(\sum_{q \in \mathcal{Q}_{\mu}} \sum_{\kappa \in \mathcal{C}_{\alpha}}\left\|P_{q} R_{\kappa} \psi_{\lambda_{1}}\right\|_{L_{t, x}^{4}}^{2}\right)^{1 / 2} \\
& \quad \lesssim \alpha^{-2 \delta}\left(\frac{\mu}{\lambda_{1}}\right)^{-2 \delta}\left(\mu \lambda_{1}\right)^{1 / 4}\left(\lambda_{1}^{-1 / 2}\left\|\psi_{\lambda_{1}}\right\|_{L_{t, x}^{4}}\right)^{\delta}\left\|\psi_{\lambda_{1}}\right\|_{V_{ \pm_{1}, M}^{2}}^{1-\delta} .
\end{aligned}
$$

On the other hand, applying the Klein-Gordon Strichartz estimate and (44), we deduce that for $\frac{10}{3} \leqslant r<4$ and $\beta=(d / \mu)^{1 / 2}$ :

$$
\begin{aligned}
& \left|\int_{\mathbb{R}^{1+3}} A_{0} d x d t\right| \\
& \quad \lesssim \alpha \sum_{\substack{\kappa, \kappa^{\prime} \in \mathcal{C}_{\mathcal{\alpha}} \\
\left| \pm_{1} \kappa- \pm_{2} \kappa^{\prime}\right| \approx \alpha}} \sum_{\substack{\kappa^{\prime \prime} \in \mathcal{C}_{\beta} \\
\left|\kappa^{\prime \prime}- \pm_{2} \kappa^{\prime}\right| \lesssim \beta}}\left\|C_{d} R_{\kappa^{\prime \prime}} \phi_{\mu}\right\|_{L_{t, x}^{r /(r-2)}}\left\|R_{\kappa} \mathcal{C}_{\ll d}^{ \pm} \psi_{\lambda_{1}}\right\|_{L_{t, x}^{r}}\left\|R_{\kappa^{\prime}} \mathcal{C}_{\ll d}^{ \pm_{2}} \varphi_{\lambda_{2}}\right\|_{L_{t, x}^{r}} \\
& \quad \lesssim\left(\frac{d}{\mu}\right)^{4 / r-1-2 \delta}\left(\frac{\mu}{\lambda_{1}}\right)^{-6 \delta} \mu^{8 / r-2}\left\|\phi_{\mu}\right\|_{L_{t, x}^{4}}^{8 / r-2}\left(\mu^{1 / 2}\left\|\phi_{\mu}\right\|_{V_{+, 1}^{2}}\right)^{3-8 / r}\left\|\psi_{\lambda_{1}}\right\|_{V_{ \pm_{1}, M}^{2}}\left\|\varphi_{\lambda_{2}}\right\|_{V_{ \pm_{2}, M}^{2}}^{2} .
\end{aligned}
$$


Combining these bounds with $r=\frac{10}{3}$ then gives, for every $0<\delta, \theta<\frac{2}{5}$,

$$
\begin{aligned}
& \left|\int_{\mathbb{R}^{1+3}} A_{0} d x d t\right| \\
& \quad \lesssim\left(\frac{d}{\mu}\right)^{-2 \delta}\left(\frac{\mu}{\lambda_{1}}\right)^{1 / 2-6(\theta+\delta)} \mu^{(2 / 5) \theta} \mathbf{A}^{\delta \theta}\left(\mu^{1 / 2}\left\|\phi_{\mu}\right\|_{V_{+, 1}^{2}}\left\|\psi_{\lambda_{1}}\right\|_{V_{ \pm_{1}, M}^{2}}\left\|\varphi_{\lambda_{2}}\right\|_{V_{ \pm_{2}, M}^{2}}\right)^{1-\delta \theta} .
\end{aligned}
$$

Summing up over $\mu^{-1} \lesssim d \lesssim \mu$ and choosing $\delta, \theta>0$ sufficiently small then gives the required estimate for the $A_{0}$ term.

To estimate the $A_{1}$ term, we again put the high modulation term into $L_{t, x}^{2}$, which gives

$$
\begin{aligned}
& \left|\int_{\mathbb{R}^{1+3}} A_{1} d x d t\right| \\
& \lesssim \alpha \sum_{\substack{\kappa, \kappa^{\prime} \in \mathcal{C}_{\alpha} \\
\left| \pm_{1} \kappa- \pm_{2} \kappa^{\prime}\right| \lesssim \alpha}} \sum_{\substack{\kappa^{\prime \prime} \in \mathcal{C}_{\beta} \\
\left|\kappa^{\prime \prime}- \pm_{2} \kappa^{\prime}\right| \lesssim \beta}}\left\|R_{\kappa^{\prime \prime}} C_{\lesssim d} \phi_{\mu}\right\|_{L_{t, x}^{4}}\left\|R_{\kappa} \mathcal{C}_{d}^{ \pm_{1}} \psi_{\lambda_{1}}\right\|_{L_{t, x}^{2}}\left\|R_{\kappa^{\prime}} \mathcal{C}_{\lesssim d}^{ \pm_{2}} \varphi_{\lambda_{2}}\right\|_{L_{t, x}^{4}} \\
& \lesssim\left(\frac{d}{\mu}\right)^{-2 \delta}\left(\frac{\mu}{\lambda_{1}}\right)^{1 / 2-6 \delta}\left(\left\|\phi_{\mu}\right\|_{L_{t, x}^{4}} \lambda_{2}^{-1 / 2}\left\|\varphi_{\lambda_{2}}\right\|_{L_{t, x}^{4}}\right)^{\delta} \\
& \cdot\left(\mu^{1 / 2}\left\|\phi_{\mu}\right\|_{V_{+, 1}^{2}}\left\|\varphi_{\lambda_{2}}\right\|_{V_{ \pm_{2}, M}^{2}}\right)^{1-\delta}\left\|\psi_{\lambda_{1}}\right\|_{V_{ \pm_{1}, M}^{2}} \cdot
\end{aligned}
$$

To gain an $L_{t, x}^{4}$ norm of $\psi_{\lambda_{1}}$, we essentially repeat the argument used in the $A_{0}$ case. More precisely, we observe that using the Klein-Gordon Strichartz estimate for $\phi_{\mu}$ and $\varphi_{\lambda_{2}}$ gives for every $\frac{10}{3} \leqslant r<4$ and $\delta>0$ :

$$
\begin{aligned}
& \left|\int_{\mathbb{R}^{1+3}} A_{1} d x d t\right| \\
& \quad \lesssim \alpha \sum_{\substack{\kappa, \kappa^{\prime} \in \mathcal{C}_{\alpha} \\
\left| \pm_{1} \kappa- \pm_{2} \kappa^{\prime}\right| \lesssim \alpha}}\left\|C_{\lesssim d} \phi_{\mu}\right\|_{L_{t, x}^{r}}\left\|C_{d} R_{\kappa} \psi_{\lambda_{1}}\right\|_{L_{t, x}^{2}}^{3-8 / r}\left\|C_{d} R_{\kappa} \psi_{\lambda_{1}}\right\|_{L_{t, x}^{4}}^{8 / r-2}\left\|C_{\lesssim d} R_{\kappa^{\prime}} \varphi_{\lambda_{2}}\right\|_{L_{t, x}^{r}} \\
& \quad \lesssim\left(\frac{d}{\mu}\right)^{4 / r-1-4 \delta}\left(\frac{\mu}{\lambda_{1}}\right)^{3 / 2-4 / r-4 \delta} \mu^{4 / r-1} \mu^{1 / 2}\left\|\phi_{\mu}\right\|_{V_{+, 1}^{2}} \\
& \quad \cdot\left(\lambda_{1}^{-1 / 2}\left\|\psi_{\lambda_{1}}\right\|_{L_{t, x}^{4}}\right)^{8 / r-2}\left\|\psi_{\lambda_{1}}\right\|_{V_{ \pm_{1}, M}^{2}}^{3-8 / r}\left\|\varphi_{\lambda_{2}}\right\|_{V_{ \pm_{2}, M}^{2},},
\end{aligned}
$$

where the square sums over caps were again controlled by using [6, Lemma 8.6]. Together with (47), this completes the proof for the $A_{1}$ component. An identical computation gives an acceptable bound for the $A_{2}$ term. Hence, by choosing $\delta$ sufficiently small and summing up over $\mu^{-1} \lesssim d \lesssim \mu$, we get the required bound in Case 1. 
Case 2: $\mu \gg \min \left\{\lambda_{1}, \lambda_{2}\right\}$ and $d \lesssim \min \left\{\lambda_{1}, \lambda_{2}\right\}$. We only consider the case $\lambda_{1} \geqslant \lambda_{2}$, as the remaining case is identical. As previously, we first estimate $A_{0}$ by placing $\phi_{\mu} \in L^{2}$ and $\psi_{\lambda_{1}}, \varphi_{\lambda_{2}} \in L^{4}$. From the resonance identities in [6, Lemma 8.7] together with (42) we obtain

$$
d \gtrsim \mathfrak{M}_{ \pm_{1}, \pm_{2}}(\xi, \eta) \gtrsim \frac{\mu^{2}}{\lambda_{2}} \theta^{2}\left(\xi-\eta, \pm_{1} \xi\right)+\lambda_{2} \theta^{2}\left( \pm_{1} \xi, \pm_{2} \eta\right)+\lambda_{2} \theta^{2}\left(\xi-\eta, \pm_{2} \eta\right)
$$

Hence, if we let $\beta=\left(d / \lambda_{2}\right)^{1 / 2}$ we obtain

$$
\begin{aligned}
& \left|\int_{\mathbb{R}^{1+3}} A_{0} d x d t\right| \\
& \quad \lesssim \beta \sum_{\substack{q, q^{\prime \prime} \in Q_{\lambda_{2}} \\
\left|q-q^{\prime \prime}\right| \approx \lambda_{2}}} \sum_{\substack{\kappa, \mathcal{K}_{1} \in \mathcal{C}_{\beta} \kappa- \pm_{2} \kappa^{\prime} \mid \lesssim \beta\\
}}\left\|P_{q^{\prime \prime}} C_{d} \phi_{\mu}\right\|_{L_{t, x}^{2}}\left\|R_{\kappa} P_{q} \psi_{\lambda_{1}}\right\|_{L_{t, x}^{4}}\left\|R_{\mathcal{K}^{\prime}} \varphi_{\lambda_{2}}\right\|_{L_{t, x}^{4}} \\
& \quad \lesssim\left(\frac{d}{\lambda_{2}}\right)^{-4 \delta}\left(\frac{\lambda_{2}}{\mu}\right)^{1 / 4-2 \delta} \mu^{1 / 2}\left\|\phi_{\mu}\right\|_{V_{+, 1}^{2}}\left(\lambda_{1}^{-1 / 2}\left\|\psi_{\lambda_{1}}\right\|_{L_{t, x}^{4}} \lambda_{2}^{-1 / 2}\left\|\varphi_{\lambda_{2}}\right\|_{L_{t, x}^{4}}\right)^{\delta} \\
& \quad \cdot\left(\left\|\psi_{\lambda_{1}}\right\|_{V_{ \pm_{1}, M}^{2}}\left\|\varphi_{\lambda_{2}}\right\|_{V_{ \pm_{2}, M}^{2}}\right)^{1-\delta}
\end{aligned}
$$

for every $0<\delta<1$. Thus we have a high-low gain provided we place the functions $\phi_{\mu}, \psi_{\lambda_{1}}$, and $\varphi_{\lambda_{2}}$ into the relevant $V^{2}$ space. On the other hand, letting $\alpha=\left(d \lambda_{2} / \mu^{2}\right)^{1 / 2}$ and applying the Klein-Gordon Strichartz estimate gives for $\frac{10}{3} \leqslant r<4$ :

$$
\begin{aligned}
& \left|\int_{\mathbb{R}^{1+3}} A_{0} d x d t\right| \\
& \quad \lesssim \beta \sum_{\substack{\kappa, \kappa^{\prime} \in \mathcal{C}_{\beta} \\
\left| \pm_{1} \kappa- \pm_{2} \kappa^{\prime}\right| \lesssim \beta}} \sum_{\substack{\kappa^{\prime \prime} \in \mathcal{C}_{\alpha} \\
\pm_{1} \kappa-\kappa^{\prime \prime} \mid \lesssim \alpha}}\left\|C_{d} R_{\kappa^{\prime \prime}} \phi_{\mu}\right\|_{L_{t, x}^{r / r-2)}}\left\|R_{\kappa} \mathcal{C}_{d}^{ \pm 1} \psi_{\lambda_{1}}\right\|_{L_{t, x}^{r}}\left\|R_{\kappa^{\prime}} \mathcal{C}_{d}^{ \pm_{2}} \varphi_{\lambda_{2}}\right\|_{L_{t, x}^{r}} \\
& \quad \lesssim\left(\frac{d}{\lambda_{2}}\right)^{4 / r-1-2 \delta}\left(\frac{\mu}{\lambda_{2}}\right)^{1+4 / r} \lambda_{2}^{8 / r-2} \\
& \quad \cdot\left(\mu^{1 / 2}\left\|\phi_{\mu}\right\|_{V_{+, 1}^{2}}\right)^{3-8 / r}\left\|\phi_{\mu}\right\|_{L_{t, x}^{4}}^{8 / r-2}\left\|\psi_{\lambda_{1}}\right\|_{V_{ \pm_{1}, M}^{2}}\left\|\varphi_{\lambda_{2}}\right\|_{V_{ \pm_{2}, M}^{2}},
\end{aligned}
$$

where we used that $\sup _{\kappa \in \mathcal{C}_{\beta}}\left\{\kappa^{\prime \prime} \in \mathcal{C}_{\alpha}:\left| \pm_{1} \kappa-\kappa^{\prime \prime}\right| \lesssim \alpha\right\} \lesssim\left(\mu / \lambda_{2}\right)^{2}$. Provided we choose $\delta>0$ sufficiently small, the above estimates and summation with respect to $\lambda_{2}^{-1} \lesssim d \lesssim \mu$ give an acceptable bound for the $A_{0}$ term.

The argument to control the $A_{1}$ term is similar. We just reverse the roles of $\phi_{\mu}$ and $\psi_{\lambda_{1}}$, and note that, with $\alpha=\left(d \lambda_{2} / \mu^{2}\right)^{1 / 2}$, 


$$
\begin{aligned}
& \left|\int_{\mathbb{R}^{1+3}} A_{1} d x d t\right| \\
& \quad \lesssim \beta \sum_{\substack{q, q^{\prime \prime} \in Q_{\lambda_{2}} \\
\left|q-q^{\prime \prime}\right| \approx \lambda_{2}}} \sum_{\substack{\kappa, \kappa^{\prime \prime} \in \mathcal{C}_{\alpha} \\
\left| \pm_{1} \kappa-\kappa^{\prime \prime}\right| \lesssim \alpha}} \sum_{\substack{\kappa^{\prime} \in \mathcal{C}_{\beta} \\
\left| \pm_{1} \kappa- \pm_{2} \kappa^{\prime}\right| \lesssim \beta}}\left\|P_{q^{\prime \prime}} R_{\kappa^{\prime \prime}} \phi_{\mu}\right\|_{L_{t, x}^{4}}\left\|R_{\kappa} P_{q} \mathcal{C}_{d}^{ \pm_{1}} \psi_{\lambda_{1}}\right\|_{L_{t, x}^{2}}\left\|R_{\kappa^{\prime}} \varphi_{\lambda_{2}}\right\|_{L_{t, x}^{4}} \\
& \quad \lesssim\left(\frac{d}{\lambda_{2}}\right)^{-4 \delta}\left(\frac{\lambda_{2}}{\mu}\right)^{1 / 4-4 \delta}\left\|\psi_{\lambda_{1}}\right\|_{V_{ \pm_{1}, M}^{2}}\left(\left\|\phi_{\mu}\right\|_{L_{t, x}^{4}} \lambda_{2}^{-1 / 2}\left\|\varphi_{\lambda_{2}}\right\|_{L_{t, x}^{4}}\right)^{\delta} \\
& \quad \cdot\left(\mu \phi^{1 / 2}\left\|\phi_{\mu}\right\|_{V_{+, 1}^{2}}\left\|\varphi_{\lambda_{2}}\right\|_{V_{ \pm_{2}, M}^{2}, M}\right)^{1-\delta} .
\end{aligned}
$$

As in the $A_{0}$ case, we can apply the Klein-Gordon Strichartz estimate to gain a positive factor of $d / \lambda_{2}$ as well as an $L_{t, x}^{4}$ factor of $\psi_{\lambda_{1}}$. Hence summing up over $\lambda_{2}^{-1} \lesssim d \ll \lambda_{2}$ gives the claimed bound for the $A_{1}$ term.

Finally to bound the $A_{2}$ component, we can either lose $\epsilon$ high derivatives, or avoid this loss by exploiting the $Y_{\lambda}^{ \pm, M}$ type norms. More precisely, using the wave Strichartz pair $(2 r /(r-1), 2 r)$ with $1 / r=1 / a-\frac{1}{2}\left(1 / a-\frac{1}{2}\right)$ and $a$ as in the definition of the $Y_{\lambda}^{ \pm, M}$ norm, we see that for any $\delta>0$ sufficiently small:

$$
\begin{aligned}
& \left|\int_{\mathbb{R}^{1+3}} A_{2} d x d t\right|
\end{aligned}
$$

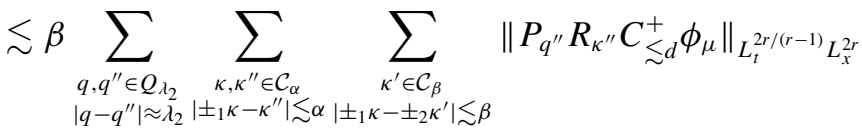

$$
\begin{aligned}
& \text { - }\left\|R_{\kappa} P_{q} \mathcal{C}_{\lesssim d}^{ \pm 1} \psi_{\lambda_{1}}\right\|_{L_{t}^{2 r /(r-1)} L_{x}^{2 r}}\left\|R_{\kappa^{\prime}} \mathcal{C}_{d}^{ \pm_{2}} \varphi_{\lambda_{2}}\right\|_{L_{t}^{r} L_{x}^{r /(r-1)}} \\
& \lesssim\left(\frac{d}{\lambda_{2}}\right)^{-b-4 \delta}\left(\frac{\lambda_{2}}{\mu}\right)^{1 / 2 a-1 / 4-4 \delta} \mathbf{A}^{\delta}\left(\mu^{1 / 2}\left\|\phi_{\mu}\right\|_{V_{+, 1}^{2}}\left\|\psi_{\lambda_{1}}\right\|_{V_{ \pm_{1}, M}^{2}}\|\varphi\|_{Y_{\lambda_{2}}^{ \pm 2}, M}\right)^{1-\delta} \text {. }
\end{aligned}
$$

Here, we have used two estimates, which require further explanation. First, $L^{r}$ interpolation together with Bernstein's inequality implies

$$
\begin{aligned}
& \left\|R_{\mathcal{K}^{\prime}} \mathcal{C}_{d}^{ \pm 2} \varphi_{\lambda_{2}}\right\|_{L_{t}^{r} L_{x}^{r /(r-1)}} \\
& \quad \lesssim\left(\frac{d}{\lambda_{2}}\right)^{-1 / 2} \lambda_{2}^{2 / r-3 / 2}\left(\lambda_{2}^{-1 / 2}\left\|R_{\kappa^{\prime}} \mathcal{C}_{d}^{ \pm 2} \varphi_{\lambda_{2}}\right\|_{L_{t, x}^{4}}\right)^{\delta_{0}}\left(d^{-1 / a}\left\|R_{\kappa^{\prime}} \mathcal{C}_{d}^{ \pm 2} \varphi_{\lambda_{2}}\right\|_{L_{t}^{a} L_{x}^{2}}\right)^{1-\delta_{0}}
\end{aligned}
$$

with $\delta_{0} \in(0,1)$ satisfying $1 / r=\delta_{0} / 4+\left(1-\delta_{0}\right) / a$. Then, by writing $C_{d}^{ \pm_{2}} \varphi_{\lambda_{2}}$ as a superposition of free waves and applying the Strichartz estimate and Hölder's inequality, we obtain

$$
\lambda_{2}^{-1 / 2}\left\|R_{\kappa^{\prime}} \mathcal{C}_{d}^{ \pm 2} \varphi_{\lambda_{2}}\right\|_{L_{t, x}^{4}} \lesssim d^{1 / a}\left\|R_{\kappa^{\prime}} \mathcal{C}_{d}^{ \pm 2} \varphi_{\lambda_{2}}\right\|_{L_{t}^{a} L_{x}^{2}}
$$


We conclude that for all $0<\delta<\delta_{0}$

$$
\left\|R_{\kappa^{\prime}} \mathcal{C}_{d}^{ \pm 2} \varphi_{\lambda_{2}}\right\|_{L_{t}^{r} L_{x}^{r /(r-1)}} \lesssim\left(\frac{d}{\lambda_{2}}\right)^{-1 / 2-b} \lambda_{2}^{1 / a-1}\left(\lambda_{2}^{-1 / 2}\left\|\varphi_{\lambda_{2}}\right\|_{L_{t, x}^{4}}\right)^{\delta}\|\varphi\|_{Y_{\lambda_{2}}^{ \pm}, M}^{1-\delta},
$$

since $R_{\kappa^{\prime}} \mathcal{C}_{d}^{ \pm 2}$ is uniformly disposable here. The second estimate used above is the following: Since $\frac{5}{3}<a<2$ we have via [6, Lemma 8.6] for every $0<\delta<\frac{1}{2}$

$$
\begin{aligned}
& \left(\sum_{q \in Q_{\lambda_{2}}} \sum_{\kappa \in \mathcal{C}_{\alpha}}\left\|R_{\kappa} P_{q} \mathcal{C}_{\lesssim d}^{ \pm{ }_{1}} \psi_{\lambda_{1}}\right\|_{L_{t}^{2 r /(r-1)} L_{x}^{2 r}}^{2 r}\right)^{1 / 2} \\
& \quad \lesssim\left(\mu \lambda_{2}\right)^{1 / 2-1 / 2 r}\left(\sum_{q \in Q_{\lambda_{2}}} \sum_{\kappa \in \mathcal{C}_{\beta}}\left(\lambda_{1}^{-1 / 2}\left\|\psi_{\lambda_{1}}\right\|_{L_{t, x}^{4}}\right)^{2 \delta}\left\|\psi_{\lambda_{1}}\right\|_{V_{ \pm_{1}, M}^{2}}^{2-2 \delta}\right)^{1 / 2} \\
& \quad \lesssim\left(\frac{\lambda_{2}}{\mu}\right)^{-2 \delta}\left(\frac{d}{\lambda_{2}}\right)^{-2 \delta}\left(\mu \lambda_{2}\right)^{3 / 8-1 / 4 a}\left(\lambda_{1}^{-1 / 2}\left\|\psi_{\lambda_{1}}\right\|_{L_{t, x}^{4}}\right)^{\delta}\left\|\psi_{\lambda_{1}}\right\|_{V_{ \pm_{1}, M}^{2}}^{1-\delta}
\end{aligned}
$$

together with a similar bound for the $\phi_{\mu}$ term. Thus summing up over $\lambda_{2}^{-1} \lesssim$ $d \lesssim \lambda_{2}$ and choosing $\delta>0$ sufficiently small (depending on both $a$ and $\varrho$ ), we get an acceptable bound for the $A_{2}$ term.

We also require a bound for the $A_{2}$ component without using the $Y_{\lambda}^{ \pm, M}$ norm. To this end, we note that for every $\delta>0$ we have the weaker bound

$$
\begin{aligned}
& \left|\int_{\mathbb{R}^{1+3}} A_{2} d x d t\right| \\
& \lesssim \beta \sum_{\substack{q, q^{\prime \prime} \in \mathcal{C}_{\lambda_{2}} \\
\left|q-q^{\prime \prime}\right| \approx \lambda_{2}}} \sum_{\substack{\kappa, \kappa^{\prime \prime} \in \mathcal{C}_{\alpha} \\
\left| \pm \pm_{1} \kappa-\kappa^{\prime \prime}\right| \lesssim \alpha}} \sum_{\substack{\kappa^{\prime} \in \mathcal{C}_{\beta} \\
\left| \pm_{1} \kappa- \pm_{2} \kappa^{\prime}\right| \lesssim \beta}}\left\|R_{\kappa^{\prime \prime}} P_{q^{\prime \prime}} C_{\lesssim d}^{+} \phi_{\mu}\right\|_{L_{t, x}^{4}} \\
& \text { - }\left\|R_{\kappa} P_{q} \mathcal{C}_{\lesssim d}^{ \pm 1} \psi_{\lambda_{1}}\right\|_{L_{t, x}^{4}}\left\|R_{\kappa^{\prime}} \mathcal{C}_{d}^{ \pm 2} \varphi_{\lambda_{2}}\right\|_{L_{t, x}^{2}} \\
& \lesssim\left(\frac{d}{\lambda_{2}}\right)^{-4 \delta}\left(\frac{\lambda_{2}}{\mu}\right)^{-4 \delta}\left(\left\|\phi_{\mu}\right\|_{L_{t, x}^{4}} \lambda_{1}^{-1 / 2}\left\|\psi_{\lambda_{1}}\right\|_{L_{t, x}^{4}}\right)^{\delta} \\
& \cdot\left(\mu^{1 / 2}\left\|\phi_{\mu}\right\|_{V_{+, 1}^{2}}\left\|\psi_{\lambda_{1}}\right\|_{V_{ \pm_{1}, M}^{2}}\right)^{1-\delta}\left\|\varphi_{\lambda_{2}}\right\|_{V_{ \pm_{2}, M}^{2}} \text {. }
\end{aligned}
$$

To gain a power of the $L_{t, x}^{4}$ norm of $\varphi_{\lambda_{2}}$, we exploit the Klein-Gordon Strichartz estimates as previously, which gives for $\frac{10}{3} \leqslant r<4$ and $\delta>0$ :

$$
\begin{aligned}
& \left|\int_{\mathbb{R}^{1+3}} A_{2} d x d t\right| \\
& \lesssim \beta \sum_{\substack{\kappa, \kappa^{\prime} \in \mathcal{C}_{\alpha} \\
\left| \pm_{1} \kappa- \pm_{2} \kappa^{\prime}\right| \lesssim \alpha}} \sum_{\substack{\kappa^{\prime} \in \mathcal{C}_{\beta} \\
\left| \pm_{1} \kappa- \pm_{2} \kappa^{\prime}\right| \lesssim \beta}}\left\|C_{\lesssim d} R_{\kappa^{\prime \prime}} \phi_{\mu}\right\|_{L_{t, x}^{r}}\left\|C_{\lesssim d} R_{\kappa} \psi_{\lambda_{1}}\right\|_{L_{t, x}^{r}}
\end{aligned}
$$




$$
\begin{aligned}
& \left\|C_{d} R_{\kappa^{\prime}} \varphi_{\lambda_{2}}\right\|_{L_{t, x}^{2}}^{3-8 / r}\left\|C_{d} R_{\kappa^{\prime}} \varphi_{\lambda_{2}}\right\|_{L_{t, x}^{4}}^{8 / r-2} \\
\lesssim & \left(\frac{d}{\lambda_{2}}\right)^{4 / r-1+\delta}\left(\frac{\lambda_{2}}{\mu}\right)^{-1 / 2-2 \delta} \lambda_{2}^{8 / r-2} \mu^{1 / 2}\left\|\phi_{\mu}\right\|_{V_{+, 1}^{2}}\left\|\psi_{\lambda_{1}}\right\|_{V_{ \pm_{1}, M}^{2}} \\
& \cdot\left(\lambda_{1}^{-1 / 2}\left\|\varphi_{\lambda_{2}}\right\|_{L_{t, x}^{4}}\right)^{8 / r-2}\left\|\varphi_{\lambda_{2}}\right\|_{V_{ \pm_{2}, M}^{2}, 3-r}^{3-8 / r}
\end{aligned}
$$

Combining these bounds and summing up with respect to $\lambda_{2}^{-1} \lesssim d \lesssim \lambda_{2}$, we obtain an acceptable contribution for the $A_{2}$ term.

Case 3: $\mu \ll \lambda_{1} \approx \lambda_{2}$ and $d \gg \mu$. To bound the $A_{0}$ component, we first observe that $\int_{\mathbb{R}^{1+3}} A_{0} \neq 0$ implies $d \approx \mathfrak{M}_{ \pm_{1}, \pm_{2}}$. In particular, since either $\mathfrak{M}_{ \pm_{1}, \pm_{2}} \lesssim \mu$ or $\mathfrak{M}_{ \pm_{1}, \pm_{2}} \approx \lambda_{1}$, the sum over the modulation is restricted to $d \gtrsim \lambda_{1}$. If we now apply Theorem 5 with $\gamma=\frac{1}{8}$ we see that

$$
\begin{aligned}
& \left|\int_{\mathbb{R}^{1+3}} A_{0} d x d t\right| \\
& \quad \lesssim\left\|C_{d}^{+} \phi_{\mu}\right\|_{L_{t, x}^{2}}\left\|P_{\mu}\left[\left(\mathcal{C}_{\ll d}^{ \pm_{1}} \psi_{\lambda_{1}}\right)^{\dagger} \gamma^{0} \mathcal{C}_{\ll d}^{ \pm_{2}} \varphi_{\lambda_{2}}\right]\right\|_{L_{t, x}^{2}} \\
& \quad \lesssim\left(\frac{d}{\lambda_{1}}\right)^{-1 / 2}\left(\frac{\mu}{\lambda_{1}}\right)^{1 / 4} \mu^{1 / 2}\left\|\phi_{\mu}\right\|_{V_{+, 1}^{2}} \\
& \quad \cdot\left(\lambda_{1}^{-1 / 2}\left\|\psi_{\lambda_{1}}\right\|_{L_{t, x}^{4}} \lambda_{1}^{-1 / 2}\left\|\varphi_{\lambda_{2}}\right\|_{L_{t, x}^{4}}\right)^{1 / 8}\left(\left\|\psi_{\lambda_{1}}\right\|_{V_{ \pm_{1}, M}^{2}}\left\|\varphi_{\lambda_{2}}\right\|_{V_{ \pm_{2}, M}^{2}}\right)^{7 / 8}
\end{aligned}
$$

On the other hand, using the Klein-Gordon Strichartz estimates, and noting that the $C_{\ll d}$ multipliers are now disposable, we have for $\frac{10}{3} \leqslant r<4$ the estimate

$$
\begin{aligned}
\left|\int_{\mathbb{R}^{1+3}} A_{0} d x d t\right| \leqslant & \left\|C_{d} \phi_{\mu}\right\|_{L_{t, x}^{r /(r-2)}}\left\|\psi_{\lambda_{1}}\right\|_{L_{t, x}^{r}}\left\|\varphi_{\lambda_{2}}\right\|_{L_{t, x}^{r}} \\
\lesssim & \left(\frac{d}{\mu}\right)^{4 / r-1} \mu^{8 / r-2}\left\|\phi_{\mu}\right\|_{L_{t, x}^{4}}^{8 / r-2} \\
& \cdot\left(\mu^{1 / 2}\left\|\phi_{\mu}\right\|_{V_{+, 1}^{2}}\right)^{3-8 / r}\left\|\psi_{\lambda_{1}}\right\|_{V_{ \pm_{1}, M}^{2}}\left\|\varphi_{\lambda_{2}}\right\|_{V_{ \pm_{2}, M}^{2}} .
\end{aligned}
$$

In particular, interpolating between these bounds and summing up over $d \gtrsim \lambda_{1}$ gives an acceptable contribution for the $A_{0}$ term.

The bound for the $A_{1}$ term is slightly different as we no longer have $\mathfrak{M}_{ \pm_{1}, \pm_{2}} \approx d$, and thus have to consider the full region $d \gg \mu$. We first deal with the region $d \gtrsim \lambda_{1}$. Here we argue as usual by controlling the integral by $L^{4} \times L^{2} \times L^{4}$, which gives for every $0<\delta \leqslant 1$ 


$$
\begin{aligned}
& \left|\int_{\mathbb{R}^{1+3}} A_{1} d x d t\right| \\
& \quad \lesssim\left\|\phi_{\mu}\right\|_{L_{t, x}^{4}} \sum_{\substack{q, q^{\prime} \in Q_{\mu} \\
\left|q-q^{\prime}\right| \lesssim \mu}}\left\|P_{q} C_{d} \psi_{\lambda_{1}}\right\|_{L_{t, x}^{2}}\left\|P_{q^{\prime}} \varphi_{\lambda_{2}}\right\|_{L_{t, x}^{4}} \\
& \quad \lesssim\left(\frac{d}{\lambda_{1}}\right)^{-1 / 2}\left(\frac{\mu}{\lambda_{1}}\right)^{1 / 4-2 \delta}\left\|\phi_{\mu}\right\|_{L_{t, x}^{4}}\left\|\psi_{\lambda_{2}}\right\|_{V_{ \pm_{1}, M}^{2}}\left(\lambda_{1}^{-1 / 2}\left\|\varphi_{\lambda_{2}}\right\|_{L_{t, x}^{4}}\right)^{\delta}\left\|\varphi_{\lambda_{2}}\right\|_{V_{ \pm_{2}, M}^{2}}^{1-\delta},
\end{aligned}
$$

where we controlled the square sum as previously via an estimate analogous to (46). To gain an $L_{t, x}^{4}$ norm of $\psi$, we again exploit the Klein-Gordon Strichartz estimate and observe that for $\frac{10}{3} \leqslant r<4$ we have

$$
\begin{aligned}
& \left|\int_{\mathbb{R}^{1+3}} A_{1} d x d t\right| \\
& \quad \lesssim\left\|\phi_{\mu}\right\|_{L_{t, x}^{r}}\left\|C_{d} \psi_{\lambda_{1}}\right\|_{L_{t, x}^{2}}^{3-8 / r}\left\|C_{d} R_{\kappa} \psi_{\lambda_{1}}\right\|_{L_{t, x}^{4}}^{8 / r-2}\left\|\varphi_{\lambda_{2}}\right\|_{L_{t, x}^{r}} \\
& \quad \lesssim\left(\frac{d}{\lambda_{1}}\right)^{4 / r-3 / 2} \lambda_{1}^{8 / r-2} \mu^{1 / 2}\left\|\phi_{\mu}\right\|_{V_{+, 1}^{2}}\left\|\varphi_{\lambda_{2}}\right\|_{V_{ \pm_{2}, M}^{2}}\left(\lambda_{1}^{-1 / 2}\left\|\psi_{\lambda_{1}}\right\|_{L_{t, x}^{4}}\right)^{8 / r-2}\left\|\psi_{\lambda_{1}}\right\|_{V_{ \pm}^{2}, M}^{3-8 / r}
\end{aligned}
$$

Combining bounds (54) and (55), and summing up over modulation, we deduce the required bound for $A_{1}$ in the region $d \gtrsim \lambda_{1}$.

We now consider the case $\mu \ll d \ll \lambda_{1}$. This implies that $\mathfrak{M}_{ \pm_{1}, \pm_{2}} \ll \lambda_{1}$, which is only possible if $\pm_{1}= \pm_{2}$. The key point is that we may now exploit the null structure in the product of the spinors $\psi$ and $\varphi$, since we gain $\theta(\xi, \eta)$, and the angle between the supports of $\widehat{\psi}$ and $\widehat{\phi}$ is less than $\mu / \lambda_{1}$. In particular, exploiting the standard null structure bound implies that we may improve (54) to

$$
\begin{aligned}
& \left|\int_{\mathbb{R}^{1+3}} A_{1} d x d t\right| \\
& \quad \lesssim\left(\frac{d}{\lambda_{1}}\right)^{-1 / 2}\left(\frac{\mu}{\lambda_{1}}\right)^{5 / 4-2 \delta}\left\|\phi_{\mu}\right\|_{L_{t, x}^{4}}\left\|\psi_{\lambda_{2}}\right\|_{V_{ \pm_{1}, M}^{2}}\left(\lambda_{1}^{-1 / 2}\left\|\varphi_{\lambda_{2}}\right\|_{L_{t, x}^{4}}\right)^{\delta}\left\|\varphi_{\lambda_{2}}\right\|_{V_{ \pm_{2}, M}^{2}}^{1-\delta} .
\end{aligned}
$$

Again combining this bound with (55), and summing up over $\mu \ll d \ll \lambda_{1}$, the required bound for the $A_{1}$ term follows. The $A_{2}$ term follows from an identical argument.

Case 4: $\mu \gtrsim \min \left\{\lambda_{1}, \lambda_{2}\right\}$ and $d \gtrsim \min \left\{\lambda_{1}, \lambda_{2}\right\}$. It is enough to consider the case $\lambda_{1} \geqslant \lambda_{2}$. To estimate the $A_{0}$ term, we first observe that as in the previous case, we may restrict the sum over modulation to $d \gtrsim \mu$. If we now observe that 


$$
\begin{aligned}
\left|\int_{\mathbb{R}^{1+3}} A_{0} d x d t\right| & \lesssim\left\|C_{d}^{+} \phi_{\mu}\right\|_{L_{t, x}^{2}}\left\|\psi_{\lambda_{1}}\right\|_{L_{t, x}^{4}}\left\|\varphi_{\lambda_{2}}\right\|_{L_{t, x}^{4}} \\
& \lesssim\left(\frac{d}{\mu}\right)^{-1 / 2}\left(\frac{\lambda_{2}}{\mu}\right)^{1 / 2} \mu^{1 / 2}\left\|\phi_{\mu}\right\|_{V_{+, 1}^{2}} \lambda_{1}^{-1 / 2}\left\|\psi_{\lambda_{1}}\right\|_{L_{t, x}^{4}} \lambda_{2}^{-1 / 2}\left\|\varphi_{\lambda_{2}}\right\|_{L_{t, x}^{4}}
\end{aligned}
$$

then, together with (53), summing up over $d \gtrsim \mu$ gives the required bound for the $A_{0}$ term.

If we suppose that $d \gtrsim \mu$, then a similar argument handles the $A_{1}$ term. Again supposing that $d \gtrsim \mu$, to bound the $A_{2}$ term, we decompose into cubes of diameter $\mu$ to obtain

$$
\begin{aligned}
& \left|\int_{\mathbb{R}^{1+3}} A_{2} d x d t\right| \\
& \quad \lesssim \sum_{\substack{q, q^{\prime} \in Q_{\lambda_{2}} \\
\left|q-q^{\prime \prime}\right| \approx \mu}}\left\|P_{q^{\prime \prime}} \phi_{\mu}\right\|_{L_{t, x}^{4}}\left\|P_{q} \psi_{\lambda_{1}}\right\|_{L_{t, x}^{4}}\left\|\mathcal{C}_{d}^{ \pm 2} \varphi_{\lambda_{2}}\right\|_{L_{t, x}^{2}} \\
& \quad \lesssim\left(\frac{d}{\mu}\right)^{-1 / 2}\left(\frac{\lambda_{2}}{\mu}\right)^{1 / 2-4 \delta}\left(\left\|\phi_{\mu}\right\|_{L_{t, x}^{4}} \lambda_{1}^{-1 / 2}\left\|\psi_{\lambda_{1}}\right\|_{L_{t, x}^{4}}\right)^{\delta} \\
& \quad \cdot\left(\mu \phi^{1 / 2}\left\|\phi_{\mu}\right\|_{V_{+, 1}^{2}}\left\|\psi_{\lambda_{1}}\right\|_{V_{ \pm_{1}, M}^{2}}\right)^{1-\delta}\left\|\varphi_{\lambda_{2}}\right\|_{V_{ \pm_{2}, M}^{2},} .
\end{aligned}
$$

An analogous estimate to (55) then gives an additional $L_{t, x}^{4}$ norm of $\varphi_{\lambda_{2}}$. This gives an acceptable bound when $d \gtrsim \mu$.

It remains to bound the $A_{1}$ and $A_{2}$ terms when $\lambda_{2} \ll d \ll \mu$. We first recall that either $\mathfrak{M}_{ \pm_{1}, \pm_{2}} \approx \lambda_{1}$ or $\mathfrak{M}_{ \pm_{1}, \pm_{2}} \lesssim \lambda_{2}$. Hence the restriction $\lambda_{2} \ll d \ll \mu$ implies that $\mathfrak{M}_{ \pm_{1}, \pm_{2}} \ll d$ and consequently, a short computation shows that at least $t w o$ of the functions $\phi, \psi$, and $\varphi$ must have large modulation. More precisely, we have the decomposition

$$
\begin{aligned}
\int_{\mathbb{R}^{1+3}} A_{1} d x d t= & \int_{\mathbb{R}^{1+3}} C_{\approx d} \phi_{\mu}\left(\mathcal{C}_{d}^{ \pm 1} \psi_{\lambda_{1}}\right)^{\dagger} \gamma^{0} \mathcal{C}_{\lesssim d}^{ \pm 2} \varphi_{\lambda_{2}} d x d t \\
& +\int_{\mathbb{R}^{1+3}} C_{\ll d} \phi_{\mu}\left(\mathcal{C}_{d}^{ \pm_{1}} \psi_{\lambda_{1}}\right)^{\dagger} \gamma^{0} \mathcal{C}_{\approx d}^{ \pm_{2}} \varphi_{\lambda_{2}} d x d t
\end{aligned}
$$

To bound the first term in (59), we observe that

$$
\begin{aligned}
& \left|\int_{\mathbb{R}^{1+3}} C_{\approx d} \phi_{\mu}\left(\mathcal{C}_{d}^{ \pm} \psi_{\lambda_{1}}\right)^{\dagger} \gamma^{0} \mathcal{C}_{\lesssim d}^{ \pm 2} \varphi_{\lambda_{2}} d x d t\right| \\
& \quad \lesssim\left\|C_{\approx d} \phi_{\mu}\right\|_{L_{t, x}^{2}}\left\|\mathcal{C}_{d}^{ \pm_{1}} \psi_{\lambda_{1}}\right\|_{L_{t, x}^{2}}\left\|\mathcal{C}_{\lesssim d}^{ \pm 2} \varphi_{\lambda_{2}}\right\|_{L_{t, x}^{\infty}} \\
& \quad \lesssim\left(\frac{d}{\lambda_{2}}\right)^{-1}\left(\frac{\lambda_{2}}{\mu}\right)^{1 / 2} \mu^{1 / 2}\left\|\phi_{\mu}\right\|_{V_{+, 1}^{2}}\left\|\psi_{\lambda_{1}}\right\|_{V_{ \pm_{1}, M}^{2}}\left\|\varphi_{\lambda_{2}}\right\|_{V_{ \pm_{2}, M}^{2}} .
\end{aligned}
$$


On the other hand, to bound the second term in (59), we decompose into cubes of size $\lambda_{2}$ and apply Bernstein's inequality, which gives for every $\epsilon>0$

$$
\begin{aligned}
& \left|\int_{\mathbb{R}^{1+3}} C_{\ll d} \phi_{\mu}\left(\mathcal{C}_{d}^{ \pm_{1}} \psi_{\lambda_{1}}\right)^{\dagger} \gamma^{0} \mathcal{C}_{\lesssim d}^{ \pm 2} \varphi_{\lambda_{2}} d x d t\right| \\
& \quad \lesssim \sum_{\substack{q, q^{\prime} \in \mathcal{Q}_{\lambda_{2}} \\
\left|q-q^{\prime}\right| \lesssim \lambda_{2}}}\left\|C_{\ll d} P_{q^{\prime}} \phi_{\mu}\right\|_{L_{t, x}^{\infty}}\left\|P_{q} \mathcal{C}_{d}^{ \pm 1} \psi_{\lambda_{1}}\right\|_{L_{t, x}^{2}\left\|\mathcal{C}_{\approx d}^{ \pm_{2}} \varphi_{\lambda_{2}}\right\|_{L_{t, x}^{2}}} \\
& \quad \lesssim\left(\frac{d}{\lambda_{2}}\right)^{-1}\left(\frac{\lambda_{2}}{\mu}\right)^{1 / 2-\epsilon} \mu^{1 / 2}\left\|\phi_{\mu}\right\|_{V_{+, 1}^{2}}\left\|\psi_{\lambda_{1}}\right\|_{V_{ \pm_{1}, M}^{2}}\left\|\varphi_{\lambda_{2}}\right\|_{V_{ \pm_{2}, M}^{2}} .
\end{aligned}
$$

Thus we deduce that for $\lambda_{2} \ll d \ll \mu$ and $\mu \approx \lambda_{1} \gtrsim \lambda_{2}$ we have

$$
\left|\int_{\mathbb{R}^{1+3}} A_{1} d x d t\right| \lesssim\left(\frac{d}{\lambda_{2}}\right)^{-1}\left(\frac{\lambda_{2}}{\mu}\right)^{1 / 4} \mu^{1 / 2}\left\|\phi_{\mu}\right\|_{V_{+, 1}^{2}}\left\|\psi_{\lambda_{1}}\right\|_{V_{ \pm_{1}, M}^{2}}\left\|\varphi_{\lambda_{2}}\right\|_{V_{ \pm_{2}, M}^{2}} .
$$

To gain powers of the $L_{t, x}^{4}$ norm, we simply use an analogous bound to (57) and (53). Thus summing up over $\lambda_{2} \ll d \ll \mu$ then gives the required bound for the $A_{1}$ term.

We now consider the $A_{2}$ term in the region $\lambda_{2} \ll d \ll \mu$. As in the argument for the $A_{1}$ term, we have the decomposition

$$
\begin{aligned}
\int_{\mathbb{R}^{1+3}} A_{2} d x d t= & \int_{\mathbb{R}^{1+3}} C_{\approx d} \phi_{\mu}\left(\mathcal{C}_{\lesssim d}^{ \pm 1} \psi_{\lambda_{1}}\right)^{\dagger} \gamma^{0} \mathcal{C}_{d}^{ \pm 2} \varphi_{\lambda_{2}} d x d t \\
& +\int_{\mathbb{R}^{1+3}} C_{\ll d} \phi_{\mu}\left(\mathcal{C}_{\approx d}^{ \pm \Psi_{1}} \psi_{\lambda_{1}}\right)^{\dagger} \gamma^{0} \mathcal{C}_{d}^{ \pm_{2}} \varphi_{\lambda_{2}} d x d t .
\end{aligned}
$$

The first term in (61) can be handled in an analogous manner to the second term in (59). Namely, decomposing into cubes and applying Bernstein's inequality gives for every $\epsilon>0$

$$
\begin{aligned}
& \left|\int_{\mathbb{R}^{1+3}} C_{\approx d} \phi_{\mu}\left(\mathcal{C}_{\lesssim d}^{ \pm 1} \psi_{\lambda_{1}}\right)^{\dagger} \gamma^{0} \mathcal{C}_{d}^{ \pm 2} \varphi_{\lambda_{2}} d x d t\right| \\
& \quad \lesssim \sum_{\substack{q, q^{\prime} \in \mathcal{Q}_{\lambda_{2}} \\
\left|q-q^{\prime}\right| \lesssim \lambda_{2}}}\left\|C_{\approx d} P_{q^{\prime}} \phi_{\mu}\right\|_{L_{t, x}^{2}}\left\|P_{q} \mathcal{C}_{d}^{ \pm_{1}} \psi_{\lambda_{1}}\right\|_{L_{t, x}^{\infty}\left\|\mathcal{C}_{\approx d}^{ \pm 2} \varphi_{\lambda_{2}}\right\|_{L_{t, x}^{2}}} \\
& \quad \lesssim\left(\frac{d}{\lambda_{2}}\right)^{-1}\left(\frac{\lambda_{2}}{\mu}\right)^{1 / 2-\epsilon} \mu^{1 / 2}\left\|\phi_{\mu}\right\|_{V_{+, 1}^{2}}\left\|\psi_{\lambda_{1}}\right\|_{V_{ \pm_{1}, M}^{2}}\left\|\varphi_{\lambda_{2}}\right\|_{V_{ \pm_{2}, M}^{2}} .
\end{aligned}
$$

Applying an identical argument to the second term in (61), we deduce that

$$
\left|\int_{\mathbb{R}^{1+3}} A_{2} d x d t\right| \lesssim\left(\frac{d}{\lambda_{2}}\right)^{-1}\left(\frac{\lambda_{2}}{\mu}\right)^{1 / 4} \mu^{1 / 2}\left\|\phi_{\mu}\right\|_{V_{+, 1}^{2}}\left\|\psi_{\lambda_{1}}\right\|_{V_{ \pm_{1}, M}^{2}}\left\|\varphi_{\lambda_{2}}\right\|_{V_{ \pm_{2}, M}^{2}} .
$$


Together with the standard bound (58) and the $A_{2}$ version of (55), after summing up over $\lambda_{2} \ll d \ll \mu$ we deduce the final bound required for the $A_{2}$ component.

6.2. Proof of Theorem 7. The first step is to obtain frequency localized versions of the required bounds. Namely, if $\varrho>0$ is sufficiently small and we take $1 / a=\frac{1}{2}+\varrho / 32$ and $b=4\left(1 / a-\frac{1}{2}\right)$, our aim is to show there exists $0<\theta_{1}<\frac{1}{4}$ such that for all $0 \leqslant \theta \leqslant \theta_{1}$ we have for the Dirac Duhamel term, the bounds

$$
\begin{aligned}
\left\|\Pi_{ \pm_{1}} P_{\lambda_{1}} \mathcal{I}^{ \pm_{1}, M}\left(\phi_{\mu} \gamma^{0} \Pi_{ \pm_{2}} \varphi_{\lambda_{2}}\right)\right\|_{V_{ \pm_{1}, M}^{2},} & \\
\lesssim & \left(\min \left\{\mu, \lambda_{2}\right\}\right)^{\varrho}\left(\frac{\min \left\{\mu, \lambda_{2}\right\}}{\max \left\{\mu, \lambda_{2}\right\}}\right)^{\varrho / 100}\left(\left\|\phi_{\mu}\right\|_{L_{t, x}^{4}} \lambda_{2}^{-1 / 2}\left\|\varphi_{\lambda_{2}}\right\|_{L_{t, x}^{4}}\right)^{\theta} \\
& \cdot\left(\mu^{1 / 2}\left\|\phi_{\mu}\right\|_{V_{+, 1}^{2}}\|\varphi\|_{F_{\lambda_{2}}^{ \pm}, M}\right)^{1-\theta}
\end{aligned}
$$

and

$$
\begin{aligned}
\| \Pi_{ \pm_{1}} & \mathcal{I}^{ \pm_{1}, M}\left(\phi_{\mu} \gamma^{0} \Pi_{ \pm_{2}} \varphi_{\lambda_{2}}\right) \|_{Y_{\lambda_{1}}^{ \pm_{1}, M}} \\
\lesssim & \left(\min \left\{\mu, \lambda_{2}\right\}\right)^{\varrho}\left(\frac{\min \left\{\mu, \lambda_{2}\right\}}{\max \left\{\mu, \lambda_{2}\right\}}\right)^{\varrho / 100}\left(\left\|\phi_{\mu}\right\|_{L_{t, x}^{4}} \lambda_{2}^{-1 / 2}\left\|\varphi_{\lambda_{2}}\right\|_{L_{t, x}^{4}}\right)^{\theta} \\
& \cdot\left(\mu^{1 / 2}\left\|\phi_{\mu}\right\|_{V_{+, 1}^{2}}\left\|\varphi_{\lambda_{2}}\right\|_{V_{ \pm_{2}, M}^{2}}\right)^{1-\theta},
\end{aligned}
$$

while for the wave Duhamel term, we have

$$
\begin{aligned}
\mu^{-1 / 2} & \left\|P_{\mu} \mathcal{I}^{+, 1}\left(\overline{\Pi_{ \pm_{1}} \psi_{\lambda_{1}}} \Pi_{ \pm_{2}} \varphi_{\lambda_{2}}\right)\right\|_{V_{+, 1}^{2}} \\
\lesssim & \left(\min \left\{\mu, \lambda_{1}, \lambda_{2}\right\}\right)^{\varrho}\left(\frac{\min \left\{\mu, \lambda_{1}, \lambda_{2}\right\}}{\max \left\{\mu, \lambda_{1}, \lambda_{2}\right\}}\right)^{\varrho / 100}\left(\lambda_{1}^{-1 / 2}\left\|\psi_{\lambda_{1}}\right\|_{L_{t, x}^{4}} \lambda_{2}^{-1 / 2}\left\|\varphi_{\lambda_{2}}\right\|_{L_{t, x}^{4}}\right)^{\theta} \\
& \cdot\left(\|\psi\|_{\left.F_{\lambda_{1}}^{ \pm_{1}, M}\|\varphi\|_{F_{\lambda_{2}}^{ \pm_{2}, M}}\right)^{1-\theta} .}\right.
\end{aligned}
$$

Assuming bounds (63), (64), and (65) for the moment, the estimates in Theorem 7 are a consequence of a straightforward summation argument. More precisely, fix $s_{0}>0$ sufficiently small. We have

$$
\begin{aligned}
& \left\|\Pi_{ \pm_{1}} \mathcal{I}^{ \pm_{1}, M}\left(\phi \gamma^{0} \Pi_{ \pm_{2}} \varphi_{\lambda_{2}}\right)\right\|_{\mathbf{V}_{ \pm_{1}, M}^{s_{0}}} \\
& \lesssim \sum_{\lambda_{1}} \lambda_{1}^{s_{0}}\left(\sum_{\substack{\mu, \lambda_{2} \\
\mu \approx \lambda_{2} \gg \lambda_{1}}}\left\|P_{\lambda_{1}} \Pi_{ \pm_{1}} \mathcal{I}^{ \pm_{1}, M}\left(\phi_{\mu} \gamma^{0} \Pi_{ \pm_{2}} \varphi_{\lambda_{2}}\right)\right\|_{V_{ \pm_{1}, M}^{2}}\right. \\
& \left.+\sum_{\substack{\mu, \lambda_{2} \\
\lambda_{1} \approx \max \left\{\mu, \lambda_{2}\right\}}}\left\|P_{\lambda_{1}} \Pi_{ \pm_{1}} \mathcal{I}^{ \pm_{1}, M}\left(\phi_{\mu} \gamma^{0} \Pi_{ \pm_{2}} \varphi_{\lambda_{2}}\right)\right\|_{V_{ \pm_{1}, M}^{2}}\right) .
\end{aligned}
$$


An application of (63) with $\varrho=\frac{100}{101} s_{0}$ gives $0<\theta_{0}<\frac{1}{2}$ such that for all $0<\theta<$ $\min \left\{\theta_{0}, \frac{1}{202}\right\}$ we have

$$
\begin{aligned}
\| & \Pi_{ \pm_{1}} \mathcal{I}^{ \pm_{1}, M}\left(\phi \gamma^{0} \Pi_{ \pm_{2}} \varphi_{\lambda_{2}}\right) \|_{\mathbf{V}_{ \pm_{1}, M}^{s_{0}}} \\
\lesssim & \left.\left(\sup _{\mu, \lambda_{2}}\left\|\phi_{\mu}\right\|_{L_{t, x}^{4}} \lambda_{2}^{-1 / 2}\left\|\varphi_{\lambda_{2}}\right\|_{L_{t, x}^{4}}\right)^{\theta}\left(\mu^{1 / 2+s_{0}}\left\|\phi_{\mu}\right\|_{V_{+, 1}^{2}} \lambda_{2}^{s_{0}}\|\varphi\|_{F_{\lambda_{2}}^{ \pm}, M}\right)^{(1-\theta)}\right) \\
& \cdot\left(\sum_{\lambda_{1}} \sum_{\mu \gg \lambda_{1}} \lambda_{1}^{-(1 / 101-2 \theta) s_{0}}\left(\frac{\mu}{\lambda_{1}}\right)^{-(102 / 101-2 \theta) s_{0}}+\sum_{\lambda_{1}} \sum_{\mu \lesssim \lambda_{1}} \lambda_{1}^{-(1 / 101-2 \theta) s_{0}}\left(\frac{\mu}{\lambda_{1}}\right)^{\theta s_{0}}\right) \\
\lesssim & \sup _{\mu, \lambda_{2}}\left(\left\|\phi_{\mu}\right\|_{L_{t, x}^{4}} \lambda_{2}^{-1 / 2}\left\|\varphi_{\lambda_{2}}\right\|_{L_{t, x}^{4}}\right)^{\theta}\left(\mu^{1 / 2+s_{0}}\left\|\phi_{\mu}\right\|_{V_{+, 1}^{2}} \lambda_{2}^{s_{0}}\|\varphi\|_{F_{\lambda_{2}}^{ \pm}, M}\right)^{(1-\theta)} .
\end{aligned}
$$

Thus we obtain (34) in the case $s=s_{0}$. The general case $s>s_{0}$ follows by using the fact that $\lambda_{1}^{s} \lesssim \lambda_{1}^{s_{0}}\left(\max \left\{\mu, \lambda_{2}\right\}\right)^{s-s_{0}}$. An identical argument using (64) gives the $\mathbf{Y}_{ \pm_{1}, M}^{s}$ bound (35). Similarly the bound (36) follows from (65).

We now turn to the proof of estimates (63), (64), and (65). It is enough to consider the case $\theta=\theta_{1}$, as the $L_{t, x}^{4}$ terms are dominated by the corresponding $V^{2}$ norms. Bounds (63) and (65) follow directly from Theorem 8 together with (24). On the other hand, the argument used to obtain (64) is slightly more involved. We start by considering the case $\mu \lesssim \lambda_{2}$. An application of the Klein-Gordon Strichartz estimate gives for every $\epsilon>0$ :

$$
\begin{aligned}
& d^{3 / 5}\left(\frac{\min \left\{d, \lambda_{1}\right\}}{\lambda_{1}}\right)^{1-3 / 5}\left\|P_{\lambda_{1}} \mathcal{C}_{d}^{ \pm_{1}, M} \mathcal{I}^{ \pm_{1}, M}\left(\phi_{\mu} \gamma^{0} \Pi_{ \pm_{2}} \varphi_{\lambda_{2}}\right)\right\|_{L_{t}^{5 / 3} L_{x}^{2}} \\
& \quad \lesssim \lambda_{1}^{-2 / 5}\left\|\left(\sum_{q \in Q_{\mu}}\left\|\phi_{\mu} P_{q} \varphi_{\lambda_{2}}\right\|_{L_{x}^{2}}^{2}\right)^{1 / 2}\right\|_{L_{t}^{5 / 3}} \\
& \quad \lesssim \lambda_{1}^{-2 / 5}\left\|\phi_{\mu}\right\|_{L_{t, x}^{10 / 3}}\left(\sum_{q \in Q_{\mu}}\left\|P_{q} \varphi_{\lambda_{2}}\right\|_{L_{t}^{10 / 3} L_{x}^{5}}^{2}\right)^{1 / 2} \\
& \quad \lesssim \lambda_{1}^{-2 / 5}\left(\mu \lambda_{2}\right)^{3 / 10}\left(\frac{\mu}{\lambda_{2}}\right)^{-\epsilon} \mu^{1 / 2}\left\|\phi_{\mu}\right\|_{V_{+, 1}^{2}}\left\|\varphi_{\lambda_{2}}\right\|_{V_{ \pm_{2}, M}^{2}} .
\end{aligned}
$$

As we may assume that $\max \left\{\mu, \lambda_{1}\right\} \approx \lambda_{2}$, by choosing $\epsilon>0$ small, we deduce that

$$
\begin{aligned}
& d^{3 / 5}\left(\frac{\min \left\{d, \lambda_{1}\right\}}{\lambda_{1}}\right)^{1-3 / 5}\left\|P_{\lambda_{1}} \mathcal{C}_{d}^{ \pm_{1}, M} \mathcal{I}_{ \pm_{1}, M}\left(\phi_{\mu} \gamma^{0} \Pi_{ \pm_{2}} \varphi_{\lambda_{2}}\right)\right\|_{L_{t}^{5 / 3} L_{x}^{2}} \\
& \quad \lesssim \mu^{1 / 5}\left(\frac{\mu}{\lambda_{2}}\right)^{1 / 11} \mu^{1 / 2}\left\|\phi_{\mu}\right\|_{V_{+, 1}^{2}}\left\|\varphi_{\lambda_{2}}\right\|_{V_{ \pm_{2}, M}^{2}} \cdot
\end{aligned}
$$


On the other hand, an application of (37) in Theorem 8 gives

$$
\begin{aligned}
d^{1 / 2} & \left\|P_{\lambda_{1}} \mathcal{C}_{d}^{ \pm_{1}, M} \mathcal{I}^{ \pm_{1}, M}\left(\phi_{\mu} \gamma^{0} \Pi_{ \pm_{2}} \varphi_{\lambda_{2}}\right)\right\|_{L_{t, x}^{2}} \\
& \lesssim\left\|P_{\lambda_{1}} \Pi_{ \pm_{1}} \mathcal{I}^{ \pm_{1}, M}\left(\phi_{\mu} \gamma^{0} \Pi_{ \pm_{2}} \varphi_{\lambda_{2}}\right)\right\|_{V_{ \pm_{1}, M}^{2}} \\
& \lesssim \mu^{\varrho / 2}\left(\frac{\mu}{\lambda_{2}}\right)^{1 / 10}\left(\left\|\phi_{\mu}\right\|_{L_{t, x}^{4}} \lambda_{2}^{-1 / 2}\left\|\varphi_{\lambda_{2}}\right\|_{L_{t, x}^{4}}\right)^{\theta_{0}}\left(\mu^{1 / 2}\left\|\phi_{\mu}\right\|_{V_{+, 1}^{2}}\left\|\varphi_{\lambda_{2}}\right\|_{V_{ \pm_{2}, M}^{2}}\right)^{1-\theta_{0}}
\end{aligned}
$$

Hence (64) in the region $\mu \lesssim \lambda_{2}$ follows by interpolating between (66) and (67) and using the condition $1 / a \stackrel{1}{=}+\varrho / 32$.

We now consider the case $\mu \gg \lambda_{2}$. For this frequency interaction, Theorem 8 requires a $Y_{\lambda_{2}}^{ \pm, M}$ norm on the right-hand side. Thus, as our goal is to obtain a bound only using the $V_{ \pm, M}^{2}$ norms, we have to work a little harder. We start by writing the product as

$$
\begin{aligned}
\phi_{\mu} \gamma^{0} \Pi_{ \pm_{2}} \varphi_{\lambda_{2}}= & \left(\phi_{\mu} \gamma^{0} \Pi_{ \pm_{2}} \varphi_{\lambda_{2}}-\sum_{d^{\prime} \lesssim \lambda_{2}} C_{\leqslant d^{\prime}}^{ \pm_{1}, M}\left(C_{\leqslant d^{\prime}} \phi_{\mu} \gamma^{0} \mathcal{C}_{d^{\prime}}^{ \pm_{2}} \varphi_{\lambda_{2}}\right)\right) \\
& +\sum_{d^{\prime} \lesssim \lambda_{2}} C_{\leqslant d^{\prime}}^{ \pm_{1}, M}\left(C_{\leqslant d^{\prime}} \phi_{\mu} \gamma^{0} \mathcal{C}_{d^{\prime}}^{ \pm_{2}} \varphi_{\lambda_{2}}\right) .
\end{aligned}
$$

The first term can be bounded by adapting the argument used in the case $\mu \lesssim \lambda_{2}$ as here (38) in Theorem 8 gives a bound without using the $Y_{\lambda_{2}}^{ \pm, M}$ norm. More precisely, letting $\beta=\left(d^{\prime} / \lambda_{2}\right)^{1 / 2}$ and exploiting the null structure, we have

$$
\begin{aligned}
& d^{3 / 5}\left(\frac{d}{\lambda_{1}}\right)^{1-3 / 5}\left\|P_{\lambda_{1}} \mathcal{C}_{d}^{ \pm 1} \mathcal{I}^{ \pm_{1}, M}\left(\sum_{d^{\prime} \lesssim \lambda_{2}} C_{\leqslant d^{\prime}}^{ \pm, M}\left(C_{\leqslant d^{\prime}} \phi_{\mu} \gamma^{0} \mathcal{C}_{d^{\prime}}^{ \pm 2} \varphi_{\lambda_{2}}\right)\right)\right\|_{L_{t}^{5 / 3} L_{x}^{2}} \\
& \lesssim \mu^{-2 / 5} \sum_{d^{\prime} \lesssim \lambda_{2}}\left\|\left(\sum_{\substack{\kappa, \kappa^{\prime} \in \mathcal{C}_{\beta} \\
\left|\kappa-\kappa^{\prime}\right| \lesssim \beta}}\left\|R_{\kappa} \Pi_{ \pm_{1}}\left(C_{\leqslant d^{\prime}} \phi_{\mu} \gamma^{0} R_{\kappa^{\prime}} \mathcal{C}_{d^{\prime}}^{ \pm 2} \varphi_{\lambda_{2}}\right)\right\|_{L_{x}^{2}}^{2}\right)^{1 / 2}\right\|_{L_{t}^{5 / 3}} \\
& \lesssim \mu^{-2 / 5} \sum_{d^{\prime} \lesssim \lambda_{2}} \beta\left\|C_{\leqslant d^{\prime}} \phi_{\mu}\right\|_{L_{t, x}^{10 / 3}}\left(\sum_{\kappa^{\prime} \in \mathcal{C}_{\beta}}\left\|R_{\kappa^{\prime}} \mathcal{C}_{d^{\prime}}^{ \pm 2} \varphi_{\lambda_{2}}\right\|_{L_{t}^{10 / 3} L_{x}^{5}}^{2}\right)^{1 / 2} \\
& \lesssim \lambda_{2}^{1 / 5}\left(\frac{\lambda_{2}}{\mu}\right)^{2 / 5} \mu^{1 / 2}\left\|\phi_{\mu}\right\|_{V_{+, 1}^{2}}\left\|\varphi_{\lambda_{2}}\right\|_{V_{ \pm_{2}, M}^{2}} .
\end{aligned}
$$

Consequently, applying a similar argument to the $\phi_{\mu} \gamma^{0} \Pi_{ \pm_{2}} \varphi_{\lambda_{2}}$ component, we deduce that

$$
\begin{aligned}
& d^{3 / 5}\left(\frac{d}{\lambda_{1}}\right)^{1-3 / 5}\left\|P_{\lambda_{1}} \mathcal{C}_{d}^{ \pm 1} \mathcal{I}^{ \pm_{1}, M}\left(\phi_{\mu} \gamma^{0} \Pi_{ \pm_{2}} \varphi_{\lambda_{2}}-\sum_{d^{\prime} \lesssim \lambda_{2}} C_{\leqslant d^{\prime}}^{ \pm_{1}, M}\left(C_{\leqslant d^{\prime}} \phi_{\mu} \gamma^{0} \mathcal{C}_{d^{\prime}}^{ \pm_{2}} \varphi_{\lambda_{2}}\right)\right)\right\|_{L_{t}^{5 / 3} L_{x}^{2}} \\
& \lesssim \lambda_{2}^{1 / 5}\left(\frac{\lambda_{2}}{\mu}\right)^{2 / 5} \mu^{1 / 2}\left\|\phi_{\mu}\right\|_{V_{+, 1}^{2}}\left\|\varphi_{\lambda_{2}}\right\|_{V_{ \pm_{2}, M}^{2}} .
\end{aligned}
$$


On the other hand, an application of Theorem 8 gives

$$
\begin{aligned}
d^{1 / 2} \| & P_{\lambda_{1}} \mathcal{C}_{d}^{ \pm_{1}} \mathcal{I}^{ \pm_{1}, M}\left(\phi_{\mu} \gamma^{0} \Pi_{ \pm_{2}} \varphi_{\lambda_{2}}-\sum_{d^{\prime} \lesssim \lambda_{2}} C_{\leqslant d^{\prime}}^{ \pm_{1}, M}\left(C_{\leqslant d^{\prime}} \phi_{\mu} \gamma^{0} \mathcal{C}_{d^{\prime}}^{ \pm_{2}} \varphi_{\lambda_{2}}\right)\right) \|_{L_{t, x}^{2}} \\
& \lesssim\left\|P_{\lambda_{1}} \Pi_{ \pm_{1}} \mathcal{I}^{ \pm_{1}, M}\left(\phi_{\mu} \gamma^{0} \Pi_{ \pm_{2}} \varphi_{\lambda_{2}}-\sum_{d^{\prime} \lesssim \lambda_{2}} C_{\leqslant d^{\prime}}^{ \pm_{1}, M}\left(C_{\leqslant d^{\prime}} \phi_{\mu} \gamma^{0} \mathcal{C}_{d^{\prime}}^{ \pm_{2}} \varphi_{\lambda_{2}}\right)\right)\right\|_{V_{ \pm_{1}, M}^{2},} \\
& \lesssim \lambda_{2}^{\rho / 2}\left(\frac{\lambda_{2}}{\mu}\right)^{1 / 10}\left(\left\|\phi_{\mu}\right\|_{L_{t, x}^{4}} \lambda_{2}^{-1 / 2}\left\|\varphi_{\lambda_{2}}\right\|_{L_{t, x}^{4}}\right)^{\theta_{0}}\left(\mu^{1 / 2}\left\|\phi_{\mu}\right\|_{V_{+, 1}^{2}}\left\|\varphi_{\lambda_{2}}\right\|_{V_{ \pm_{2}, M}^{2}}\right)^{1-\theta_{0}}
\end{aligned}
$$

and therefore interpolating as before gives the required bound for the first term in the decomposition (68). It remains to bound the second term in (68). Let $1<$ $r<a$. Exploiting the null structure and decomposing $\phi_{\mu}$ into caps of size $\alpha=$ $\left(d^{\prime} \lambda_{2} / \mu^{2}\right)^{1 / 2}$ and $\varphi_{\lambda_{2}}$ into caps of size $\beta=\left(d^{\prime} / \lambda_{2}\right)^{1 / 2}$, we deduce that for all $0<$ $\theta<\frac{1}{4}$ and $\max \left\{d, \lambda_{2}^{-1}\right\} \lesssim d^{\prime} \lesssim \lambda_{2}$ we have

$$
\begin{aligned}
& \left\|P_{\lambda_{1}} \mathcal{C}_{d}^{ \pm_{1}} C_{\leqslant d^{\prime}}^{ \pm_{1}, M}\left(C_{\leqslant d^{\prime}} \phi_{\mu} \gamma^{0} \mathcal{C}_{d^{\prime}}^{ \pm 2} \varphi_{\lambda_{2}}\right)\right\|_{L_{t}^{a} L_{x}^{2}} \\
& \lesssim d^{1 / r-1 / a} \|\left(\sum_{q \in Q_{\lambda_{2}}} \sum_{\substack{\kappa, \kappa^{\prime \prime} \in \mathcal{C}_{\alpha} \\
\left|\kappa-\kappa^{\prime \prime}\right| \lesssim \alpha}} \sum_{\substack{\kappa^{\prime} \in \mathcal{C}_{\beta} \\
\left|\kappa-\kappa^{\prime}\right| \lesssim \beta}}\right. \\
& \text { - } \left.\left\|P_{\lambda_{1}} R_{\kappa} \Pi_{ \pm_{1}}\left(C_{\leqslant d^{\prime}} R_{\kappa^{\prime \prime}} P_{q} \phi_{\mu} \gamma^{0} \mathcal{C}_{d^{\prime}}^{ \pm 2} R_{\kappa^{\prime}} \varphi_{\lambda_{2}}\right)\right\|_{L_{x}^{2}}^{2}\right)^{1 / 2} \|_{L_{t}^{r}} \\
& \lesssim d^{1 / r-1 / a} \beta\left(\sum_{q \in Q_{\lambda_{2}}} \sum_{\kappa^{\prime \prime} \in \mathcal{C}_{\alpha}}\left\|C_{\leqslant d^{\prime}} R_{\kappa^{\prime \prime}} P_{q} \phi_{\mu}\right\|_{L_{t}^{2 r} L_{x}^{2 r /(r-1)}}^{2}\right)^{1 / 2}\left(\sum_{\kappa^{\prime} \in \mathcal{C}_{\beta}}\left\|\mathcal{C}_{d^{\prime}}^{ \pm 2} R_{\kappa^{\prime}} \varphi_{\lambda_{2}}\right\|_{L_{t, x}^{2 r}}^{2}\right)^{1 / 2} \\
& \lesssim d^{1 / r-1 / a} \mu^{1-1 / r}\left(\frac{\lambda_{2}}{\mu}\right)^{3 / 2-3 / 2 r-6 \theta}\left(\frac{d^{\prime}}{\lambda_{2}}\right)^{1-1 / r-2 \theta} \\
& \text { - }\left(\left\|\phi_{\mu}\right\|_{L_{t, x}^{4}} \lambda_{2}^{-1 / 2}\left\|\varphi_{\lambda_{2}}\right\|_{L_{t, x}^{4}}\right)^{\theta}\left(\mu^{1 / 2}\left\|\phi_{\mu}\right\|_{V_{+, m}^{2}}\left\|\varphi_{\lambda_{2}}\right\|_{V_{ \pm_{2}, M}^{2}}\right)^{1-\theta},
\end{aligned}
$$

where we used the bounds

$$
\begin{aligned}
& \left(\sum_{\kappa^{\prime \prime} \in \mathcal{C}_{\alpha}} \sum_{q \in \mathcal{Q}_{\lambda_{2}}}\left\|C_{\leqslant d^{\prime}} R_{\kappa^{\prime \prime}} P_{q} \phi_{\mu}\right\|_{L_{t}^{2 r} L_{x}^{2 r}}^{2 r(r-1)}\right)^{1 / 2} \\
& \lesssim \lambda_{2}^{1 / r-1 / 2} \alpha^{-2 \theta}\left(\frac{\lambda_{2}}{\mu}\right)^{1 / 2-1 / 2 r-4 \theta}\left\|\phi_{\mu}\right\|_{L_{t, x}^{4}}^{\theta}\left(\mu^{1 / 2}\left\|\phi_{\mu}\right\|_{V_{+, m}^{2}}\right)^{1-\theta}
\end{aligned}
$$

and

$$
\left(\sum_{\kappa^{\prime} \in \mathcal{C}_{\beta}}\left\|\mathcal{C}_{d^{\prime}}^{ \pm 2} R_{\kappa^{\prime}} \varphi_{\lambda_{2}}\right\|_{L_{t, x}^{2 r}}^{2}\right)^{1 / 2} \lesssim \beta^{-\theta} \lambda_{2}^{1-1 / r}\left(d^{\prime}\right)^{1 / 2-1 / r}\left(\lambda_{2}^{-1 / 2}\left\|\varphi_{\lambda_{2}}\right\|_{L_{t, x}^{4}}\right)^{\theta}\left\|\varphi_{\lambda_{2}}\right\|_{V_{ \pm_{2}, M}^{2}}^{1-\theta},
$$


which, similar to (46), hold for all sufficiently small $\theta>0$ and follow from $L^{p}$ interpolation, Lemma 3, an application of Hölder's inequality, and the square sum bound for $V^{2}$. An application of (43) implies that after restricting the output to modulation $d$, the sum over the modulation is only over the region $\max \left\{d, \lambda_{2}^{-1}\right\} \lesssim$ $d^{\prime} \lesssim \lambda_{2}$. Consequently, summing up (69) we deduce that for $1<r<a$ and $\theta>0$ sufficiently small

$$
\begin{aligned}
& \left\|P_{\lambda_{1}} \mathcal{C}_{d}^{ \pm_{1}} \mathcal{I}^{ \pm_{1}, M}\left(\sum_{d^{\prime} \lesssim \lambda_{2}} C_{\leqslant d^{\prime}}^{ \pm_{1}, M}\left(C_{\leqslant d^{\prime}} \phi_{\mu} \gamma^{0} \mathcal{C}_{d^{\prime}}^{ \pm_{2}} \varphi_{\lambda_{2}}\right)\right)\right\|_{L_{t}^{a} L_{x}^{2}} \\
& \lesssim d^{-1} \quad \sum \quad\left\|P_{\lambda_{1}} \mathcal{C}_{d}^{ \pm 1}\left(C_{\leqslant d^{\prime}} \phi_{\mu} \gamma^{0} \mathcal{C}_{d^{\prime}}^{ \pm 2} \varphi_{\lambda_{2}}\right)\right\|_{L_{t}^{a} L_{x}^{2}} \\
& \max \left\{d, \lambda_{2}^{-1}\right\} \lesssim d^{\prime} \lesssim \lambda_{2} \\
& \lesssim d^{1 / r-1 / a-1} \mu^{1-1 / r}\left(\frac{\lambda_{2}}{\mu}\right)^{3 / 2-3 / 2 r-6 \theta} \sum_{\max \left\{d, \lambda_{2}^{-1}\right\} \lesssim d^{\prime} \lesssim \lambda_{2}}\left(\frac{d^{\prime}}{\lambda_{2}}\right)^{1-1 / r-2 \theta} \\
& \cdot\left(\left\|\phi_{\mu}\right\|_{L_{t, x}^{4}} \lambda_{2}^{-1 / 2}\left\|\varphi_{\lambda_{2}}\right\|_{L_{t, x}^{4}}\right)^{\theta}\left(\mu^{1 / 2}\left\|\phi_{\mu}\right\|_{V_{+, m}^{2}}\left\|\varphi_{\lambda_{2}}\right\|_{V_{ \pm_{2}, M}^{2}}\right)^{1-\theta} \\
& \lesssim d^{-1 / a}\left(\frac{d}{\mu}\right)^{1 / r-1}\left(\frac{\lambda_{2}}{\mu}\right)^{1-1 / r} \\
& \cdot\left(\left\|\phi_{\mu}\right\|_{L_{t, x}^{4}} \lambda_{2}^{-1 / 2}\left\|\varphi_{\lambda_{2}}\right\|_{L_{t, x}^{4}}\right)^{\theta}\left(\mu^{1 / 2}\left\|\phi_{\mu}\right\|_{V_{+, m}^{2}}\left\|\varphi_{\lambda_{2}}\right\|_{V_{ \pm_{2}, M}^{2}}\right)^{1-\theta} \text {. }
\end{aligned}
$$

Therefore, taking $1 / r=1-4\left(1 / a-\frac{1}{2}\right)$ we obtain (64). This completes the proof of Theorem 7.

\section{Multilinear estimates in the critical case}

In this section, we consider the scale-invariant regime with a small amount of angular regularity. Here, after rescaling, we have $m=1$ and $M>0$.

THEOREM 9. Let $M>0$ and $\sigma>0$. There exist $0<\theta<1,1<a<2$, and $b>0$ such that for all $s \geqslant 0$

$$
\begin{aligned}
\| \Pi_{ \pm_{1}} & \mathcal{I}^{ \pm_{1}, M}\left(\phi \gamma^{0} \Pi_{ \pm_{2}} \varphi\right) \|_{\mathbf{V}_{ \pm_{1}, M}^{s, \sigma}} \\
\lesssim & \left(\sup _{\mu, \lambda_{2} \geqslant 1}\left\|\phi_{\mu}\right\|_{\mathbf{D}_{\sigma}^{s}}\left\|\varphi_{\lambda_{2}}\right\|_{\mathbf{D}_{\sigma}^{-1 / 2}}\right)^{\theta}\left(\|\phi\|_{\mathbf{V}_{+, 1}^{s+1 / 2, \sigma}}\|\varphi\|_{\mathbf{F}_{ \pm_{2}, M}^{0, \sigma}}\right)^{1-\theta} \\
& +\left(\sup _{\mu, \lambda_{2} \geqslant 1}\left\|\phi_{\mu}\right\|_{\mathbf{D}_{\sigma}^{0}}\left\|\varphi_{\lambda_{2}}\right\|_{\mathbf{D}_{\sigma}^{s-1 / 2}}\right)^{\theta}\left(\|\phi\|_{\mathbf{V}_{+, 1}^{1 / 2, \sigma}}\|\varphi\|_{\mathbf{F}_{ \pm_{2}, M}^{s, \sigma}}\right)^{1-\theta}
\end{aligned}
$$


and

$$
\begin{aligned}
& \left\|\Pi_{ \pm_{1}} \mathcal{I}^{ \pm_{1}, M}\left(\phi \gamma^{0} \Pi_{ \pm_{2}} \varphi\right)\right\|_{\mathbf{Y}_{ \pm_{1}, M}^{s, \sigma}} \\
& \quad \lesssim\left(\sup _{\mu, \lambda_{2} \geqslant 1}\left\|\phi_{\mu}\right\|_{\mathbf{D}_{\sigma}^{s}}\left\|\varphi_{\lambda_{2}}\right\|_{\mathbf{D}_{\sigma}^{-1 / 2}}\right)^{\theta}\left(\|\phi\|_{\mathbf{V}_{+, 1}^{s+1 / 2, \sigma}}\|\varphi\|_{\mathbf{V}_{ \pm_{2}, M}^{0, \sigma}}\right)^{1-\theta} \\
& \quad+\left(\sup _{\mu, \lambda_{2} \geqslant 1}\left\|\phi_{\mu}\right\|_{\mathbf{D}_{\sigma}^{0}}\left\|\varphi_{\lambda_{2}}\right\|_{\mathbf{D}_{\sigma}^{s-1 / 2}}\right)^{\theta}\left(\|\phi\|_{\mathbf{V}_{+, 1}^{1 / 2, \sigma}}\|\varphi\|_{\mathbf{V}_{ \pm_{2}, M}^{s, \sigma}}\right)^{1-\theta} .
\end{aligned}
$$

Similarly,

$$
\begin{aligned}
\left\|\langle\nabla\rangle^{-1} \mathcal{I}^{+, 1}\left(\overline{\Pi_{ \pm_{1}} \psi} \Pi_{ \pm_{2}} \varphi\right)\right\|_{\mathbf{V}_{+, 1}^{s+1 / 2, \sigma}} \\
\quad \lesssim\left(\sup _{\lambda_{1}, \lambda_{2} \geqslant 1}\left\|\psi_{\lambda_{1}}\right\|_{\mathbf{D}_{\sigma}^{s-1 / 2}}\left\|\varphi_{\lambda_{2}}\right\|_{\mathbf{D}_{\sigma}^{-1 / 2}}\right)^{\theta}\left(\|\psi\|_{\mathbf{F}_{ \pm_{1}, M}^{s, \sigma}}\|\varphi\|_{\mathbf{F}_{ \pm_{2}, M}^{0, \sigma}}\right)^{1-\theta} \\
\quad+\left(\sup _{\lambda_{1}, \lambda_{2} \geqslant 1}\left\|\psi_{\lambda_{1}}\right\|_{\mathbf{D}_{\sigma}^{-1 / 2}}\left\|\varphi_{\lambda_{2}}\right\|_{\mathbf{D}_{\sigma}^{s-1 / 2}}\right)^{\theta}\left(\|\psi\|_{\mathbf{F}_{ \pm_{1}, M}^{0, \sigma}}\|\varphi\|_{\mathbf{F}_{ \pm_{2}, M}^{s, \sigma}}\right)^{1-\theta} .
\end{aligned}
$$

The proof will be postponed to Section 7.2.

7.1. The trilinear estimate. To obtain the $L_{t, x}^{4}$ norms in Theorem 9 , we use the following consequence of Theorem 6 , which gives the required $L_{t, x}^{4}$ at a cost of modulation and high-low factors. However, as we have room in our estimates, we can always absorb a small power of this loss elsewhere, which is sufficient to obtain the required Strichartz norms in Theorem 9.

LEMMA 5. Let $d>0$ and for $j=1,2,3$, let $m_{j}>0$ and $\lambda_{j}, N_{j} \geqslant 1$. If $\sigma>0$ there exists $\theta>0$ such that

$$
\begin{aligned}
& \left|\int_{\mathbb{R}^{1+3}} C_{d}^{ \pm_{1}, m_{1}} u_{\lambda_{1}, N_{1}} v_{\lambda_{2}, N_{2}} w_{\lambda_{3}, N_{3}} d x d t\right| \\
& \quad \lesssim N_{\min }^{\sigma}\left(\frac{\min \left\{d, \lambda_{1}\right\}}{\lambda_{1}}\right)^{-1 / 2-\sigma} \lambda_{\min }^{-1}\left(\left\|u_{\lambda_{1}, N_{1}}\right\|_{L_{t, x}^{4}}\left\|v_{\lambda_{2}, N_{3}}\right\|_{L_{t, x}^{4}}\left\|w_{\lambda_{3}, N_{3}}\right\|_{L_{t, x}^{4}}\right)^{\theta} \\
& \quad \cdot\left(\left(\lambda_{1} \lambda_{2} \lambda_{3}\right)^{1 / 2}\left\|u_{\lambda_{1}, N_{1}}\right\|_{V_{ \pm_{1}, m_{1}}^{2}}\left\|v_{\lambda_{2}, N_{2}}\right\|_{V_{ \pm_{2}, m_{2}}^{2}}\left\|w_{\lambda_{3}, N_{3}}\right\|_{V_{ \pm_{3}, m_{3}}^{2}}\right)^{1-\theta},
\end{aligned}
$$

where $\lambda_{\min }=\min \left\{\lambda_{1}, \lambda_{2}, \lambda_{3}\right\}$ and $N_{\min }=\min \left\{N_{1}, N_{2}, N_{3}\right\}$.

Proof. We first observe that an application of Hölder's inequality gives

$$
\begin{aligned}
& \left|\int_{\mathbb{R}^{1+3}} C_{d}^{ \pm_{1}, m_{1}} u_{\lambda_{1}, N_{1}} v_{\lambda_{2}, N_{2}} w_{\lambda_{3}, N_{3}} d x d t\right| \\
& \quad \leqslant\left\|C_{d}^{ \pm_{1}, m_{1}} u_{\lambda_{1}, N_{1}}\right\|_{L_{t, x}^{2}}\left\|v_{\lambda_{2}, N_{2}}\right\|_{L_{t, x}^{4}}\left\|w_{\lambda_{3}, N_{3}}\right\|_{L_{t, x}^{4}}
\end{aligned}
$$




$$
\begin{aligned}
& \lesssim d^{-1 / 2}\left\|u_{\lambda_{1}, N_{1}}\right\|_{V_{ \pm_{1}, m_{1}}^{2}}\left\|v_{\lambda_{2}, N_{2}}\right\|_{L_{t, x}^{4}}\left\|w_{\lambda_{3}, N_{3}}\right\|_{L_{t, x}^{4}} \\
& \lesssim\left(\frac{\min \left\{d, \lambda_{1}\right\}}{\lambda_{1}}\right)^{-1 / 2} \lambda_{\min }^{-1} \lambda_{1}^{1 / 2}\left\|u_{\lambda_{1}, N_{1}}\right\|_{V_{ \pm_{1}, m_{1}}^{2}}\left\|v_{\lambda_{2}, N_{2}}\right\|_{L_{t, x}^{4}}\left\|w_{\lambda_{3}, N_{3}}\right\|_{L_{t, x}^{4}} .
\end{aligned}
$$

Thus it only remains to show we can gain a power of the $L_{t, x}^{4}$ norm of $u_{\lambda_{1}, N_{1}}$. To this end, we first consider the case where $N_{2}=N_{\min }$. An application of the wave Strichartz estimate with angular regularity in Lemma 3 gives for $3<p<4$ :

$$
\begin{aligned}
& \left|\int_{\mathbb{R}^{1+3}} C_{d}^{ \pm_{1}, m_{1}} u_{\lambda_{1}, N_{1}} v_{\lambda_{2}, N_{2}} w_{\lambda_{3}, N_{3}} d x d t\right| \\
& \leqslant \\
& \quad\left\|C_{d}^{ \pm_{1}, m_{1}} u_{\lambda_{1}, N_{1}}\right\|_{L_{t, x}^{4}}^{4 / p-1}\left\|C_{d}^{ \pm_{1}, m_{1}} u_{\lambda_{1}, N_{1}}\right\|_{L_{t, x}^{2}}^{2-4 / p}\left\|v_{\lambda_{2}, N_{2}}\right\|_{L_{t, x}^{p}}\left\|w_{\lambda_{3}, N_{3}}\right\|_{L_{t, x}^{4}} \\
& \quad \lesssim\left(\frac{\min \left\{d, \lambda_{1}\right\}}{\lambda_{1}}\right)^{-5 / 2(4 / p-1)}\left\|u_{\lambda_{1}, N_{1}}\right\|_{L_{t, x}^{4}}^{4 / p-1}\left(d^{-1 / 2}\left\|u_{\lambda_{1}, N_{1}}\right\|_{V_{ \pm_{1}, m_{1}}^{2}}\right)^{2-4 / p} \\
& \quad \cdot N_{2} \lambda_{2}^{3 / 2-4 / p}\left\|v_{\lambda_{2}, N_{2}}\right\|_{V_{ \pm_{2}, m_{2}}^{2}} \lambda_{3}^{1 / 2}\left\|w_{\lambda_{3}, N_{3}}\right\|_{V_{ \pm_{3}, m_{3}}^{2}} \\
& \lesssim\left(\frac{\min \left\{d, \lambda_{1}\right\}}{\lambda_{1}}\right)^{-3 / 2} \frac{N_{\min }}{\lambda_{\min }}\left\|u_{\lambda_{1}, N_{1}}\right\|_{L_{t, x}^{4}}^{4 / p-1}\left(\lambda_{1}^{1 / 2}\left\|u_{\lambda_{1}, N_{1}}\right\|_{V_{ \pm_{1}, m_{1}}^{2}}\right)^{2-4 / p} \\
& \quad \cdot \lambda_{2}^{1 / 2}\left\|v_{\lambda_{2}, N_{2}}\right\|_{V_{ \pm_{2}, m_{2}}^{2}} \lambda_{3}^{1 / 2}\left\|w_{\lambda_{3}, N_{3}}\right\|_{V_{ \pm_{3}, m_{3}}^{2}},
\end{aligned}
$$

where to remove the $C_{d}^{ \pm_{1}, m_{1}}$ multiplier from the $L_{t, x}^{4}$ norm, we let $\alpha=$ $\left(\min \left\{d, \lambda_{1}\right\} / \lambda_{1}\right)^{1 / 2}$ and apply Lemma 2 to deduce that

$$
\begin{aligned}
\left\|C_{d}^{ \pm{ }_{1}, m_{1}} u_{\lambda_{1}, N_{1}}\right\|_{L_{t, x}^{4}} & \leqslant \sum_{\kappa \in \mathcal{C}_{\alpha}} \sum_{q \in \mathcal{Q}_{\alpha \lambda^{2}}}\left\|C_{d}^{ \pm_{1}, m_{1}} R_{\kappa} P_{q} u_{\lambda_{1}, N_{1}}\right\|_{L_{t, x}^{4}} \\
& \lesssim \alpha^{-2}\left((\alpha \lambda)^{-3}+1\right)\left\|u_{\lambda_{1}, N_{1}}\right\|_{L_{t, x}^{4}} \\
& \lesssim\left(\frac{\min \left\{d, \lambda_{1}\right\}}{\lambda_{1}}\right)^{-5 / 2}\left\|u_{\lambda_{1}, N_{1}}\right\|_{L_{t, x}^{4}} .
\end{aligned}
$$

If we combine (74) and (75) we deduce that

$$
\begin{aligned}
\mid \int_{\mathbb{R}^{1+3}} & C_{d}^{ \pm_{1}, m_{1}} u_{\lambda_{1}, N_{1}} v_{\lambda_{2}, N_{2}} w_{\lambda_{3}, N_{3}} d x d t \mid \\
\lesssim & {\left[\left(\frac{\min \left\{d, \lambda_{1}\right\}}{\lambda_{1}}\right)^{-1 / 2} \lambda_{\min }^{-1} \lambda_{1}^{1 / 2}\left\|u_{\lambda_{1}, N_{1}}\right\|_{V_{ \pm_{1}, m_{1}}^{2}}\left\|v_{\lambda_{2}, N_{2}}\right\|_{L_{t, x}^{4}}\left\|w_{\lambda_{3}, N_{3}}\right\|_{L_{t, x}^{4}}\right]^{1-\sigma} } \\
\cdot & {\left[\left(\frac{\min \left\{d, \lambda_{1}\right\}}{\lambda_{1}}\right)^{-3 / 2} \lambda_{\min }^{-1} N_{\min }\left\|u_{\lambda_{1}, N_{1}}\right\|_{L_{t, x}^{4}}^{4 / p-1}\right.} \\
& \left.\cdot\left(\lambda_{1}^{1 / 2}\left\|u_{\lambda_{1}, N_{1}}\right\|_{V_{ \pm_{1}, m_{1}}^{2}}\right)^{2-4 / p} \lambda_{2}^{1 / 2}\left\|v_{\lambda_{2}, N_{2}}\right\|_{V_{ \pm_{2}, m_{2}}^{2}} \lambda_{3}^{1 / 2}\left\|w_{\lambda_{3}, N_{3}}\right\|_{V_{ \pm_{3}, m_{3}}^{2}}\right]^{\sigma}
\end{aligned}
$$




$$
\begin{aligned}
\lesssim & N_{\min }^{\sigma}\left(\frac{\min \left\{d, \lambda_{1}\right\}}{\lambda_{1}}\right)^{-3 / 2} \lambda_{\min }^{-1}\left(\left\|u_{\lambda_{1}, N_{1}}\right\|_{L_{t, x}^{4}}\left\|v_{\lambda_{2}, N_{3}}\right\|_{L_{t, x}^{4}}\left\|w_{\lambda_{3}, N_{3}}\right\|_{L_{t, x}^{4}}\right)^{\sigma(4 / p-1)} \\
& \cdot\left(\left(\lambda_{1} \lambda_{2} \lambda_{3}\right)^{1 / 2}\left\|u_{\lambda_{1}, N_{1}}\right\|_{V_{ \pm_{1}, m_{1}}^{2}}\left\|v_{\lambda_{2}, N_{2}}\right\|_{V_{ \pm_{2}, m_{2}}^{2}}\left\|w_{\lambda_{3}, N_{3}}\right\|_{V_{ \pm_{3}, m_{3}}^{2}}\right)^{1-\sigma(4 / p-1)}
\end{aligned}
$$

as required. A similar argument gives the case $N_{3}=N_{\text {min }}$. Thus it remains to consider $N_{1}=N_{\min }$. It is also straightforward to deal with the case $\lambda_{2} \approx 1$, since we may then apply the Klein-Gordon Strichartz estimate. More precisely, an application of Lemma 4 to $v_{\lambda_{2}, N_{2}}$ together with $L_{t, x}^{q}$ interpolation, gives for $\frac{10}{3} \leqslant p<4$ :

$$
\begin{aligned}
& \left|\int_{\mathbb{R}^{1+3}} C_{d}^{ \pm_{1}, m_{1}} u_{\lambda_{1}, N_{1}} v_{\lambda_{2}, N_{2}} w_{\lambda_{3}, N_{3}} d x d t\right| \\
& \quad \leqslant\left\|C_{d}^{ \pm_{1}, m_{1}} u_{\lambda_{1}, N_{1}}\right\|_{L_{t, x}^{4}}^{4 / p-1}\left\|C_{d}^{ \pm_{1}, m_{1}} u_{\lambda_{1}, N_{1}}\right\|_{L_{t, x}^{2}}^{2-4 / p}\left\|v_{\lambda_{2}, N_{2}}\right\|_{L_{t, x}^{p}}\left\|w_{\lambda_{3}, N_{3}}\right\|_{L_{t, x}^{4}} \\
& \quad \lesssim\left(\frac{\min \left\{d, \lambda_{1}\right\}}{\lambda_{1}}\right)^{-(5 / 2)(4 / p-1)} \lambda_{3}^{1 / 2}\left\|u_{\lambda_{1}, N_{1}}\right\|_{L_{t, x}^{4}}^{4 / p-1}\left(d^{-1 / 2}\left\|u_{\lambda_{1}, N_{1}}\right\|_{V_{ \pm_{1}, m_{1}}^{2}}\right)^{2-4 / p} \\
& \quad \cdot\left\|v_{\lambda_{2}, N_{2}}\right\|_{V_{ \pm_{2}, m_{2}}^{2}}\left\|w_{\lambda_{3}, N_{3}}\right\|_{V_{ \pm_{3}, m_{3}}^{2}} \\
& \quad \lesssim\left(\frac{\min \left\{d, \lambda_{1}\right\}}{\lambda_{1}}\right)^{3 / 2-8 / p} \lambda_{\min }^{-1}\left\|u_{\lambda_{1}, N_{1}}\right\|_{L_{t, x}^{4}}^{4 / p-1}\left(\lambda_{1}^{1 / 2}\left\|u_{\lambda_{1}, N_{1}}\right\|_{V_{ \pm_{1}, m_{1}}^{2}}\right)^{2-4 / p} \\
& \quad \cdot \lambda_{2}^{1 / 2}\left\|v_{\lambda_{2}, N_{2}}\right\|_{V_{ \pm_{2}, m_{2}}^{2}} \lambda_{3}^{1 / 2}\left\|w_{\lambda_{3}, N_{3}}\right\|_{V_{ \pm_{3}, m_{3}}^{2}},
\end{aligned}
$$

where we applied bound (76). Combining (74) and (77) we obtain the required bound in the case where $\lambda_{2} \approx 1$. A similar argument gives the case $\lambda_{3} \approx 1$.

It remains to gain a power of the $L^{4}$ norm of $u_{\lambda_{1}, N_{1}}$, in the case where $N_{\min }=N_{1}$ and $\lambda_{2}, \lambda_{3} \gg 1$. Clearly we may also assume that $\lambda_{2} \geqslant \lambda_{3}$. In this region we apply the bilinear restriction estimate to deduce the required bound. The first step is to observe that, as either $\lambda_{1} \ll \lambda_{2} \approx \lambda_{3}$ or $\lambda_{1} \approx \max \left\{\lambda_{2}, \lambda_{3}\right\}$, an angular Whitney type decomposition gives the identity

$$
\begin{aligned}
& \int_{\mathbb{R}^{1+3}} C_{d}^{ \pm_{1}, m_{1}} u_{\lambda_{1}, N_{1}} v_{\lambda_{2}, N_{2}} w_{\lambda_{3}, N_{3}} d x d t \\
& =\sum_{\substack { 1 / \lambda_{3} \lesssim \ell \lesssim \max \left\{1, \lambda_{1} / \lambda_{3}\right\} \\
\begin{subarray}{c}{\kappa, \kappa^{\prime} \in \mathcal{C}_{\ell} \\
\left| \pm_{2} \kappa- \pm_{3} \kappa^{\prime}\right| \approx \ell{ 1 / \lambda _ { 3 } \lesssim \ell \lesssim \operatorname { m a x } \{ 1 , \lambda _ { 1 } / \lambda _ { 3 } \} \\
\begin{subarray} { c } { \kappa , \kappa ^ { \prime } \in \mathcal { C } _ { \ell } \\
| \pm _ { 2 } \kappa - \pm _ { 3 } \kappa ^ { \prime } | \approx \ell } }\end{subarray}} \int_{\mathbb{R}^{1+3}} C_{d}^{ \pm_{1}, m_{1}} u_{\lambda_{1}, N_{1}} R_{\kappa} v_{\lambda_{2}, N_{2}} R_{\kappa^{\prime}} w_{\lambda_{3}, N_{3}} d x d t \\
& \quad+\sum_{\substack{\kappa, \kappa^{\prime} \in \mathcal{C}_{1 / \lambda_{3}} \\
\left| \pm_{2} \kappa- \pm_{3} \kappa^{\prime}\right| \lesssim 1 / \lambda_{3}}} \int_{\mathbb{R}^{1+3}} C_{d}^{ \pm_{1}, m_{1}} u_{\lambda_{1}, N_{1}} R_{\kappa} v_{\lambda_{2}, N_{2}} R_{\kappa^{\prime}} w_{\lambda_{3}, N_{3}} d x d t
\end{aligned}
$$


To estimate the first term in (78), we note that as we may restrict the support of $\widehat{u}_{\lambda_{1}, N_{1}}$ to lie in the set

$$
\left\{-(\xi+\eta)\left|\xi \in \kappa, \eta \in \kappa^{\prime},\right| \xi\left|\approx \lambda_{2},\right| \eta \mid \approx \lambda_{3}\right\},
$$

we have for $\kappa, \kappa^{\prime} \in \mathcal{C}_{\ell}$ with $\left| \pm_{2} \kappa- \pm_{3} \kappa^{\prime}\right| \approx \ell$ :

$$
\begin{aligned}
& \int_{\mathbb{R}^{1+3}} C_{d}^{ \pm_{1}, m_{1}} u_{\lambda_{1}, N_{1}} R_{\kappa} v_{\lambda_{2}, N_{2}} R_{\kappa^{\prime}} w_{\lambda_{3}, N_{3}} d x d t \\
& =\sum_{\kappa^{\prime \prime} \in \mathcal{E}} \int_{\mathbb{R}^{1+3}} C_{d}^{ \pm_{1}, m_{1}} R_{\kappa^{\prime \prime}} u_{\lambda_{1}, N_{1}} R_{\kappa} v_{\lambda_{2}, N_{2}} R_{\kappa^{\prime}} w_{\lambda_{3}, N_{3}} d x d t,
\end{aligned}
$$

where $\mathcal{E}$ is the set of all $\kappa^{\prime \prime} \in \mathcal{C}_{\ell\left(\lambda_{3} / \lambda_{1}\right)}$ satisfying $\left.\min \left\{\left|\kappa^{\prime \prime}-\kappa\right|,\left|\kappa^{\prime \prime}+\kappa\right|\right\} \lesssim \ell\left(\lambda_{3} / \lambda_{1}\right)\right\}$. Notice that we have $\# \mathcal{E} \lesssim 1$. Consequently, applying Hölder's inequality, the angular concentration bound (28), and Theorem 6, we deduce that for $\kappa, \kappa^{\prime} \in \mathcal{C}_{\ell}$ with $\left| \pm_{2} \kappa- \pm_{3} \kappa^{\prime}\right| \approx \ell$ and $\frac{3}{2}<p<2$ we have

$$
\begin{aligned}
& \left|\int_{\mathbb{R}^{1+3}} C_{d}^{ \pm_{1}, m_{1}} u_{\lambda_{1}, N_{1}} R_{\kappa} v_{\lambda_{2}, N_{2}} R_{\kappa^{\prime}} w_{\lambda_{3}, N_{3}} d x d t\right| \\
& \quad \lesssim\left(\sup _{\kappa^{\prime \prime} \in \mathcal{C}_{\ell\left(\lambda_{3} / \lambda_{1}\right)}}\left\|C_{d}^{ \pm_{1}, m_{1}} R_{\kappa^{\prime \prime}} u_{\lambda_{1}, N_{1}}\right\|_{L_{t, x}^{4}}^{4(1 / p-1 / 2)}\left\|C_{d}^{ \pm_{1}, m_{1}} R_{\kappa^{\prime \prime}} u_{\lambda_{1}, N_{1}}\right\|_{L_{t, x}^{2}}^{3-4 / p}\right) \\
& \quad \cdot\left\|R_{\kappa} v_{\lambda_{2}, N_{2}} R_{\kappa^{\prime}} w_{\lambda_{3}, N_{3}}\right\|_{L_{t, x}^{p}} \\
& \lesssim N_{1}^{\sigma}\left(\ell \frac{\lambda_{3}}{\lambda_{1}}\right)^{\sigma} \ell^{2-4 / p} \lambda_{3}^{7 / 2-5 / p-\epsilon} \lambda_{2}^{1 / q-1 / 2+\epsilon}\left\|C_{d}^{ \pm_{1}, m_{1}} u_{\lambda_{1}, N_{1}}\right\|_{L_{t, x}^{4}}^{4(1 / p-1 / 2)} \\
& \quad \cdot\left(d^{-1 / 2}\left\|u_{\lambda_{1}, N_{1}}\right\|_{V_{ \pm_{1}, m_{1}}^{2}}\right)^{3-4 / p} \cdot\left\|R_{\kappa} v_{\lambda_{2}, N_{2}}\right\|_{V_{ \pm_{2}, m_{2}}^{2}}\left\|R_{\kappa^{\prime}} w_{\lambda_{3}, N_{3}}\right\|_{V_{ \pm_{3}, m_{3}}^{2}} \\
& \lesssim \ell^{\sigma+2-4 / p} N_{1}^{\sigma}\left(\frac{\min \left\{d, \lambda_{1}\right\}}{\lambda_{1}}\right)^{7 / 2-8 / p} \lambda_{\min }^{-1}\left\|u_{\lambda_{1}, N_{1}}\right\|_{L_{t, x}^{4}}^{4(1 / p-1 / 2)} \\
& \quad \cdot\left(\lambda_{1}^{1 / 2}\left\|u_{\lambda_{1}, N_{1}}\right\|_{V_{ \pm_{1}, m_{1}}^{2}}\right)^{3-4 / p} \cdot \lambda_{2}^{1 / 2}\left\|R_{\kappa} v_{\lambda_{2}, N_{2}}\right\|_{V_{ \pm_{2}, m_{2}}^{2}} \lambda_{3}^{1 / 2}\left\|R_{\kappa^{\prime}} w_{\lambda_{3}, N_{3}}\right\|_{V_{ \pm_{3}, m_{3}}^{2}},
\end{aligned}
$$

where we again applied bound (76) to dispose of the $C_{d}$ multiplier. Hence summing up over caps, letting $1 / p=\frac{1}{2}+\sigma / 8$, and applying the square sum bound in $V^{2}$, we deduce that

$$
\begin{aligned}
& \sum_{\substack{1 / \lambda_{3} \lesssim \ell \lesssim \max \left\{1, \lambda_{1} / \lambda_{3}\right\} \\
\left| \pm_{2} \kappa-\kappa_{3} \kappa^{\prime}\right| \approx \ell}}\left|\int_{\mathbb{R}^{1+3}} C_{d}^{ \pm_{1}, m_{1}} u_{\lambda_{1}, N_{1}} R_{\kappa} v_{\lambda_{2}, N_{2}} R_{\kappa^{\prime}} w_{\lambda_{3}, N_{3}} d x d t\right| \\
\lesssim & N_{1}^{\sigma}\left(\frac{\min \left\{d, \lambda_{1}\right\}}{\lambda_{1}}\right)^{-1 / 2-\sigma} \lambda_{\min }^{-1}\left\|u_{\lambda_{1}, N_{1}}\right\|_{L_{t, k}^{4}}^{\sigma / 2}\left(\lambda_{1}^{1 / 2}\left\|u_{\lambda_{1}, N_{1}}\right\|_{V_{ \pm_{1}, m_{1}}^{2}}\right)^{1-\sigma / 2} \\
\cdot & \lambda_{2}^{1 / 2}\left\|R_{\kappa} v_{\lambda_{2}, N_{2}}\right\|_{V_{ \pm_{2}, m_{2}}^{2}} \lambda_{3}^{1 / 2}\left\|R_{\kappa^{\prime}} w_{\lambda_{3}, N_{3}}\right\|_{V_{ \pm_{3}, m_{3}}^{2}} .
\end{aligned}
$$


Together with (74), we obtain an acceptable bound for the first term in (78). To bound the second term in (78), we apply a similar argument together with the Klein-Gordon Strichartz estimate to deduce that for $\frac{5}{3}<p<2$ we have

$$
\begin{aligned}
& \sum_{\substack{\kappa, \kappa^{\prime} \in \mathcal{C}_{1 / \lambda_{3}} \\
\left| \pm_{2} \kappa- \pm_{3} \kappa^{\prime}\right| \lesssim 1 / \lambda_{3}}}\left|\int_{\mathbb{R}^{1+3}} C_{d}^{ \pm_{1}, m_{1}} u_{\lambda_{1}, N_{1}} R_{\kappa} v_{\lambda_{2}, N_{2}} R_{\kappa^{\prime}} w_{\lambda_{3}, N_{3}} d x d t\right| \\
& \lesssim \sum_{\substack{\kappa, \kappa^{\prime} \in \mathcal{C}_{1 / \lambda_{3}} \\
\left| \pm_{2} \kappa- \pm_{3} \kappa^{\prime}\right| \lesssim 1 / \lambda_{3}}} \sup _{\kappa^{\prime \prime} \in \mathcal{C}_{\ell\left(\lambda_{3} / \lambda_{1}\right)}}\left\|C_{d}^{ \pm_{1}, m_{1}} R_{\kappa^{\prime \prime}} u_{\lambda_{1}, N_{1}}\right\|_{L_{t, x}^{4}}^{4(1 / p-1 / 2)}\left\|C_{d}^{ \pm_{1}, m_{1}} R_{\kappa^{\prime \prime}} u_{\lambda_{1}, N_{1}}\right\|_{L_{t, x}^{2}}^{3-4 / p} \\
& \quad \cdot\left\|R_{\kappa} v_{\lambda_{2}, N_{2}}\right\|_{L_{t, x}^{2 p}}^{2 p}\left\|R_{\kappa^{\prime}} w_{\lambda_{3}, N_{3}}\right\|_{L_{t, x}^{2 p}}^{2 p} \\
& \lesssim N_{1}^{\sigma}\left(\frac{\min \left\{d, \lambda_{1}\right\}}{\lambda_{1}}\right)^{7 / 2-8 / p} \lambda_{1}^{8(1 / p-1 / 2)-\sigma} \lambda_{\min }^{-1}\left\|u_{\lambda_{1}, N_{1}}\right\|_{L_{t, x}^{4}}^{4(1 / p-1 / 2)} \\
& \quad \cdot\left(\lambda_{1}^{-1 / 2}\left\|u_{\lambda_{1}, N_{1}}\right\|_{V_{ \pm_{1}, m_{1}}^{2}}\right)^{3-4 / p} \cdot \lambda_{2}^{1 / 2}\left\|v_{\lambda_{2}, N_{2}}\right\|_{V_{ \pm_{2}, m_{2}}^{2}} \lambda_{3}^{1 / 2}\left\|w_{\lambda_{3}, N_{3}}\right\|_{V_{ \pm_{3}, N_{3}}^{2}} .
\end{aligned}
$$

Choosing $1 / p=\frac{1}{2}+\sigma / 8$ as before and combining the resulting bound with (74), the lemma follows.

We now give the proof of the main step in the proof of Theorem 9.

THEOREM 10. Let $M>\frac{1}{2}, 0<\varrho \ll 1, \frac{5}{3}<a<2$, and $0<b<\varrho / 4$. Define

$$
\mathbf{A}=\left\|\phi_{\mu, N}\right\|_{L_{t, x}^{4}} \lambda_{1}^{-1 / 2}\left\|\psi_{\lambda_{1}, N_{1}}\right\|_{L_{t, x}^{4}} \lambda_{2}^{-1 / 2}\left\|\varphi_{\lambda_{2}, N_{2}}\right\|_{L_{t, x}^{4}} .
$$

There exists $\theta_{0} \in(0,1)$ such that, if $\mu \lesssim \lambda_{1} \sim \lambda_{2}$ we have

$$
\begin{aligned}
& \left|\int_{\mathbb{R}^{3+1}} \phi_{\mu, N} \overline{\Pi_{ \pm_{1}} \psi_{\lambda_{1}, N_{1}}} \Pi_{ \pm_{2}} \varphi_{\lambda_{2}, N_{2}} d x d t\right| \\
& \quad \lesssim N_{\min }^{\varrho}\left(\frac{\mu}{\lambda_{1}}\right)^{1 / 10} \mathbf{A}^{\theta_{0}}\left(\mu^{1 / 2}\left\|\phi_{\mu, N}\right\|_{V_{+, 1}^{2}}\left\|\psi_{\lambda_{1}, N_{1}}\right\|_{V_{ \pm_{1}, M}^{2}}\left\|\varphi_{\lambda_{2}, N_{2}}\right\|_{V_{ \pm_{2}, M}^{2}}\right)^{1-\theta_{0}} .
\end{aligned}
$$

On the other hand, when $\mu \sim \lambda_{1} \gg \lambda_{2}$ we have

$$
\begin{aligned}
& \left|\int_{\mathbb{R}^{3+1}} \phi_{\mu, N} \overline{\Pi_{ \pm_{1}} \psi_{\lambda_{1}, N_{1}}} \Pi_{ \pm_{2}} \varphi_{\lambda_{2}, N_{2}}-\sum_{\lambda_{2}^{-1} \lesssim d \lesssim \lambda_{2}} C_{\leqslant d} \phi_{\mu, N} \overline{\mathcal{C}_{\leqslant d}^{ \pm_{1}} \psi_{\lambda_{1}, N_{1}}} \mathcal{C}_{d}^{ \pm_{2}} \varphi_{\lambda_{2}, N_{2}} d x d t\right| \\
& \quad \lesssim N_{\min }^{\varrho}\left(\frac{\lambda_{2}}{\mu}\right)^{1 / 10} \mathbf{A}^{\theta_{0}}\left(\mu^{1 / 2}\left\|\phi_{\mu, N}\right\|_{V_{+, 1}^{2}}\left\|\psi_{\lambda_{1}, N_{1}}\right\|_{V_{ \pm_{1}, M}^{2}}\left\|\varphi_{\lambda_{2}, N_{2}}\right\|_{V_{ \pm_{2}, M}^{2}}\right)^{1-\theta_{0}}
\end{aligned}
$$


and

$$
\begin{aligned}
& \sum_{\lambda_{2}^{-1} \lesssim d \lesssim \lambda_{2}}\left|\int_{\mathbb{R}^{3+1}} C_{\leqslant d} \phi_{\mu, N} \overline{\mathcal{C}_{\leqslant d}^{ \pm}{ }_{\lambda_{1}, N_{1}}} \mathcal{C}_{d}^{ \pm_{2}} \varphi_{\lambda_{2}, N_{2}} d x d t\right| \\
& \lesssim\left(\min \left\{N, N_{1}\right\}\right)^{\varrho}\left(\frac{\lambda_{2}}{\mu}\right)^{1 / 10} \mathbf{A}^{\theta_{0}}\left(\mu^{1 / 2}\left\|\phi_{\mu, N}\right\|_{V_{+, 1}^{2}}\left\|\psi_{\lambda_{1}, N_{1}}\right\|_{V_{ \pm_{1}, M}^{2}}\left\|\varphi_{\lambda_{2}, N_{2}}\right\|_{V_{ \pm_{2}, M}^{2}}\right)^{1-\theta_{0}} .
\end{aligned}
$$

Moreover, if we also use the $Y^{ \pm, M}$ norm, we have

$$
\begin{aligned}
& \sum_{\lambda_{2}^{-1} \lesssim d \lesssim \lambda_{2}}\left|\int_{\mathbb{R}^{3+1}} C_{\leqslant d} \phi_{\mu, N} \overline{\mathcal{C}_{\leqslant d}^{ \pm 1}} \psi_{\lambda_{1}, N_{1}} \mathcal{C}_{d}^{ \pm_{2}} \varphi_{\lambda_{2}, N_{2}} d x d t\right| \\
& \lesssim N_{\min }^{\varrho}\left(\frac{\lambda_{2}}{\mu}\right)^{(1 / 4)(1 / a-1 / 2)} \mathbf{A}^{\theta_{0}}\left(\mu^{1 / 2}\left\|\phi_{\mu, N}\right\|_{V_{+, 1}^{2}}\left\|\psi_{\lambda_{1}, N_{1}}\right\|_{V_{ \pm_{1}, M}^{2}}\left\|\varphi_{N_{2}}\right\|_{Y_{\lambda_{2}}^{ \pm}, M}\right)^{1-\theta_{0}} .
\end{aligned}
$$

Analogous bounds hold in the case $\lambda_{1} \ll \lambda_{2}$.

Proof. As in the proof of Theorem 8, we begin by decomposing

$$
\phi_{\mu}\left(\Pi_{ \pm_{1}} \psi_{\lambda_{1}}\right)^{\dagger} \gamma^{0} \Pi_{ \pm_{2}} \varphi_{\lambda_{2}}=\sum_{d} A_{0}+A_{1}+A_{2}
$$

where

$$
\begin{aligned}
& A_{0}=C_{d} \phi_{\mu}\left(\mathcal{C}_{\ll d}^{ \pm_{1}} \psi_{\lambda_{1}}\right)^{\dagger} \gamma^{0} \mathcal{C}_{\ll d}^{ \pm_{2}} \varphi_{\lambda_{2}}, \\
& A_{1}=C_{\lesssim d} \phi_{\mu}\left(\mathcal{C}_{d}^{ \pm_{1}} \psi_{\lambda_{1}}\right)^{\dagger} \gamma^{0} \mathcal{C}_{\lesssim d}^{ \pm_{2}} \varphi_{\lambda_{2}}, \\
& A_{2}=C_{\lesssim d} \phi_{\mu}\left(\mathcal{C}_{\lesssim d}^{ \pm_{1}} \psi_{\lambda_{1}}\right)^{\dagger} \gamma^{0} \mathcal{C}_{d}^{ \pm_{2}} \varphi_{\lambda_{2}},
\end{aligned}
$$

and consider separately the small modulation cases:

$\mu \ll \lambda_{1} \approx \lambda_{2} \quad$ and $\quad d \lesssim \mu, \quad \mu \gtrsim \min \left\{\lambda_{1}, \lambda_{2}\right\} \quad$ and $\quad d \lesssim \min \left\{\lambda_{1}, \lambda_{2}\right\}$ and the high modulation case:

$$
d \gg \min \left\{\mu, \lambda_{1}, \lambda_{2}\right\} \text {. }
$$

Note that bound (43) does not hold in the case $M \leqslant \frac{1}{2}$, which is admissible now.

Case 1: $\mu \ll \lambda_{1} \approx \lambda_{2}$ and $d \lesssim \mu$. From [6, (8-16)] we have the bound $\left|\int A_{0} d x d t\right| \lesssim\left(\frac{d}{\mu}\right)^{1 / 8-\epsilon}\left(\frac{\mu}{\lambda_{1}}\right)^{-1} N_{\min }^{1 / 4} \mu^{1 / 2}\left\|\phi_{\mu, N}\right\|_{V_{+, 1}^{2}}\left\|\psi_{\lambda_{1}, N_{1}}\right\|_{V_{ \pm_{1}, M}^{2}}\left\|\varphi_{\lambda_{2}, N_{2}}\right\|_{V_{ \pm_{2}, M}^{2},}$. 
Combining this bound with Lemma 5 , choosing $\epsilon>0$ sufficiently small, and summing up over $d \lesssim \mu$, then gives $\theta>0$ such that

$$
\begin{aligned}
& \sum_{d \lesssim \mu}\left|\int_{\mathbb{R}^{1+3}} A_{0} d x d t\right| \\
& \quad \lesssim N_{\min }^{\varrho}\left(\frac{\mu}{\lambda_{2}}\right)^{1 / 4} \mathbf{A}^{\theta}\left(\mu^{1 / 2}\left\|\phi_{\mu, N}\right\|_{V_{+, 1}^{2}}\left\|\psi_{\lambda_{1}, N_{1}}\right\|_{V_{ \pm_{1}, M}^{2}}\left\|\varphi_{\lambda_{2}, N_{2}}\right\|_{V_{ \pm_{2}, M}^{2}}\right)^{1-\theta} .
\end{aligned}
$$

We now turn to the bound for the $A_{1}$ term. From [6, (8-18)] we have for every $\epsilon>0$ :

$$
\begin{aligned}
& \left|\int A_{1} d x d t\right| \\
& \quad \lesssim\left(\frac{d}{\mu}\right)^{1 / 8-\epsilon}\left(\frac{\mu}{\lambda_{1}}\right)^{-1 / 2} N_{\min }^{1 / 4} \mu^{1 / 2}\left\|\phi_{\mu, N}\right\|_{V_{+, 1}^{2}}\left\|\psi_{\lambda_{1}, N_{1}}\right\|_{V_{ \pm_{1}, M}^{2},}\left\|\varphi_{\lambda_{2}, N_{2}}\right\|_{V_{ \pm_{2}, M}^{2}} .
\end{aligned}
$$

Again combining this bound with a small power of the estimate from Lemma 5, we get an acceptable contribution for the $A_{1}$ term. The proof for the $A_{2}$ term is identical.

Case 2: $\mu \gtrsim \min \left\{\lambda_{1}, \lambda_{2}\right\}$ and $\left(\min \left\{\lambda_{1}, \lambda_{2}\right\}\right)^{-1} \lesssim d \lesssim \min \left\{\lambda_{1}, \lambda_{2}\right\}$. We may assume that $\lambda_{1} \geqslant \lambda_{2}$. From [6, (8-23)], we have for every $\epsilon>0$ :

$$
\begin{aligned}
& \left|\int_{\mathbb{R}^{1+3}} A_{0} d x d t\right|+\left|\int_{\mathbb{R}^{1+3}} A_{1} d x d t\right| \\
& \quad \lesssim\left(\frac{d}{\lambda_{2}}\right)^{1 / 8-\epsilon} N_{\min }^{1 / 4} \mu^{1 / 2}\left\|\phi_{\mu, N}\right\|_{V_{+, 1}^{2}}\left\|\psi_{\lambda_{1}, N_{1}}\right\|_{V_{ \pm_{1}, M}^{2}}\left\|\varphi_{\lambda_{2}, N_{2}}\right\|_{V_{ \pm_{2}, M}^{2}} .
\end{aligned}
$$

An application of Lemma 5, summing up over $\lambda_{2}^{-1} \lesssim d \lesssim \lambda_{2}$, then gives an acceptable bound for both $A_{0}$ and $A_{1}$.

It remains to bound the $A_{2}$ term. From [6, (8-25)] we have

$$
\begin{aligned}
& \left|\int_{\mathbb{R}^{1+3}} A_{2} d x d t\right| \\
& \quad \lesssim\left(\frac{d}{\lambda_{2}}\right)^{1 / 4-\epsilon}\left(\frac{\lambda_{2}}{\mu}\right)^{1 / 4-\epsilon} \min \left\{N, N_{1}\right\} \mu^{1 / 2}\left\|\phi_{\mu, N}\right\|_{V_{+, 1}^{2}}\left\|\psi_{\lambda_{1}, N_{1}}\right\|_{V_{ \pm_{1}, M}^{2}}\left\|\varphi_{\lambda_{2}, N_{2}}\right\|_{V_{ \pm_{2}, M}^{2}} .
\end{aligned}
$$

As previously, together with Lemma 5, this is enough to deduce the required estimates. The remaining case, where $N_{2}=N_{\min }$, requires the use of the $Y_{\lambda_{2}}^{ \pm_{2}, M}$ norm. To this end, a similar argument to (51) implies that if we let $\beta=\left(d / \lambda_{2}\right)^{1 / 2}$, 
then for every $\epsilon>0$ by using the angular concentration bound on $\varphi_{\lambda_{2}, N_{2}}$ we have

$$
\begin{aligned}
& \left|\int_{\mathbb{R}^{1+3}} A_{2} d x d t\right| \\
& \lesssim \sum_{\substack{q, q^{\prime \prime} \in Q_{\lambda_{2}} \\
\left|q-q^{\prime \prime}\right| \approx \lambda_{2}}} \sum_{\substack{\kappa, \kappa^{\prime} \kappa^{\prime \prime} \in \mathcal{C}_{\beta} \\
\left| \pm_{1} \kappa-\kappa^{\prime \prime}\right| \lesssim \beta,\left| \pm_{1} \kappa- \pm_{2} \kappa^{\prime}\right| \lesssim \beta}} \beta\left\|P_{q^{\prime \prime}} R_{\kappa^{\prime \prime}} C_{\lesssim_{d}}^{+} \phi_{\mu, N}\right\|_{L_{t}^{2 a /(a-1)} L_{x}^{2 a}} \\
& \text { - }\left\|R_{\kappa} P_{q} \mathcal{C}_{\lesssim d}^{ \pm_{1}} \psi_{\lambda_{1}, N_{1}}\right\|_{L_{t}^{2 a /(a-1)} L_{x}^{2 a}}\left\|R_{\kappa^{\prime}} \mathcal{C}_{d}^{ \pm_{2}} \varphi_{\lambda_{2}, N_{2}}\right\|_{L_{t}^{a} L_{x}^{a /(a-1)}} \\
& \lesssim\left(\frac{d}{\lambda_{2}}\right)^{\varrho / 2-b-\epsilon}\left(\frac{\lambda_{2}}{\mu}\right)^{1 / 2 a-1 / 4-\epsilon} N_{2}^{\varrho} \mu^{1 / 2}\left\|\phi_{\mu, N}\right\|_{V_{+, 1}^{2}}\left\|\psi_{\lambda_{1}, N_{1}}\right\|_{V_{ \pm_{1}, M}^{2}}\left\|H_{N_{2}} \varphi\right\|_{Y_{\lambda_{2}}^{ \pm}, M} .
\end{aligned}
$$

Therefore, since $1 / a=\frac{1}{2}+\varrho / 16$, provided we choose $\epsilon>0$ sufficiently small, by combining the above estimate with Lemma 5 we get the claimed bound for the $A_{2}$ component.

Case 3: $\mu \gtrsim \min \left\{\lambda_{1}, \lambda_{2}\right\}$ and $d \ll\left(\min \left\{\lambda_{1}, \lambda_{2}\right\}\right)^{-1}$. We now turn our attention to the resonant region where we no longer have a lower bound on $d$. It is worth noting that in this region the proof deviates somewhat from the argument in [6], as here we obtain an improvement in the amount of angular regularity required.

If $\mu \gg \min \left\{\lambda_{1}, \lambda_{2}\right\}$, [6, Lemma 8.7] implies that

$$
\mathfrak{M}_{ \pm_{1}, \pm_{2}} \gtrsim\left(\min \left\{\lambda_{1}, \lambda_{2}\right\}\right)^{-1}
$$

which is ruled out in Case 3. Hence from now on we may assume $\mu \approx \lambda_{1} \approx \lambda_{2}$. If $\pm_{1}= \pm_{2}$, or $\left( \pm_{1}, \pm_{2}\right)=(-,+)$, or $M>\frac{1}{2}[6$, Lemma 8.7] implies (83) again, so that it remains to consider $\left( \pm_{1}, \pm_{1}\right)=(+,-)$ and we are either in the weakly resonant regime $M=\frac{1}{2}$ or the strongly resonant case $0<M<\frac{1}{2}$. In order to treat this case, we use

$$
\begin{aligned}
& \mathfrak{M}_{+,-}(\xi, \eta) \\
& \quad \approx \frac{1}{\langle\xi\rangle+\langle\eta\rangle}\left|M^{2} \frac{(|\xi|-|\eta|)^{2}}{\langle\xi\rangle_{M}\langle\eta\rangle_{M}+|\xi||\eta|+M^{2}}+\right| \xi|| \eta\left|+\xi \cdot \eta+\frac{4 M^{2}-1}{2}\right| \\
& \quad \gtrsim \frac{1}{\langle\eta\rangle}\left|\frac{(|\xi|-M|\xi-\eta|)^{2}}{\langle\xi\rangle_{M}\langle\xi-\eta\rangle+|\xi||\xi-\eta|+M}+\right| \xi|| \xi-\eta\left|-\xi \cdot(\xi-\eta)+\frac{2 M-1}{2}\right|
\end{aligned}
$$

from [6, Lemma 8.7]. We start by considering the case $M=\frac{1}{2}$. The key observation (originally made in [6]) is that the null structure now acts at 
all scales $0<d<\min \left\{\lambda_{1}, \lambda_{2}\right\}$. More precisely, (84) and (31) imply that for $|\xi| \approx|\eta| \approx \mu$ :

$$
\left|\Pi_{+}(\xi) \gamma^{0} \Pi_{-}(\eta)\right|^{2} \lesssim\left|\frac{|| \xi|-| \eta||}{\mu^{2}}+\theta(\xi,-\eta)\right|^{2} \lesssim \mu^{-1} \mathfrak{M}_{+,-}(\xi, \eta),
$$

which we exploit via (30). In particular, letting $\beta=(d / \mu)^{1 / 2}$, using (84) and the $L_{t, x}^{4}$ Strichartz bound in Lemma 3 we obtain

$$
\begin{aligned}
& \left|\int_{\mathbb{R}^{1+3}} A_{0} d x d t\right| \\
& \quad \lesssim \beta \sum_{\substack{\kappa, \kappa^{\prime}, \kappa^{\prime \prime} \in \mathcal{C}_{\beta} \\
\left|\kappa+\kappa^{\prime}\right|,\left|\kappa-\kappa^{\prime \prime}\right| \lesssim \beta}} \sum_{\substack{q, q^{\prime} \in \mathcal{Q}_{\mu^{2} \beta} \\
\left|q-q^{\prime}\right| \mu^{2} \beta}}\left\|R_{\kappa^{\prime \prime}} C_{d} \phi_{\mu, N}\right\|_{L_{t, x}^{2}}\left\|R_{\kappa} P_{q} \psi_{\lambda_{1}, N_{1}}\right\|_{L_{t, x}^{4}}\left\|R_{\kappa^{\prime}} P_{q^{\prime}} \varphi_{\lambda_{2}, N_{2}}\right\|_{L_{t, x}^{4}} \\
& \quad \lesssim(\beta \mu)^{1 / 4} \mu^{1 / 2}\left\|\phi_{\mu, N}\right\|_{V_{+, 1}^{2}}\left\|\psi_{\lambda_{1}, N_{1}}\right\|_{V_{ \pm_{1}, M}^{2}}\left\|\varphi_{\lambda_{2}, N_{2}}\right\|_{V_{ \pm_{2}, M}^{2}} .
\end{aligned}
$$

Together with Lemma 5 , and as the sum over modulation $0<d \lesssim \mu^{-1}$ is bounded, this gives control over the trilinear product when $M=\frac{1}{2}$. The arguments for the $A_{1}$ and $A_{2}$ terms are essentially identical.

It remains to consider the fully resonant case $0<M<\frac{1}{2}$. In this regime the null structure no longer gives any gain at modulation scales $d \ll \mu^{-1}$. Consequently, if we followed the argument used in the $M=\frac{1}{2}$ case, we would not be able to sum up over modulation scales. Instead, our goal will be to simply estimate the remaining trilinear term

$$
\int_{\mathbb{R}^{1+3}} C_{\ll \mu^{-1}} \phi_{\mu, N} \overline{\mathcal{C}_{\ll \mu^{-1}}^{+} \psi_{\lambda_{1}, N_{1}}} \mathcal{C}_{\ll \mu^{-1}}^{-} \varphi_{\lambda_{2}, N_{2}} d x d t
$$

directly. The key observation, which was exploited in [6], is that in this trilinear interaction the three waves are already transverse, and thus we can apply the bilinear restriction estimates contained in Theorem 6 . The argument is as follows. We first observe that, by Lemma 3 and $L^{p}$ interpolation, for every $\frac{10}{3}<1 / r<\frac{5}{14}$ there exists $\theta>0$ such that

$$
\left\|\phi_{\mu, N}\right\|_{L_{t, x}^{r}} \lesssim \mu^{3 / 14} N^{2 / 5}\left\|\phi_{\mu, N}\right\|_{L_{t, x}^{4}}^{\theta}\left\|\phi_{\mu, N}\right\|_{V_{+, 1}^{2}}^{1-\theta} .
$$

Interpolating with the trivial $L_{t}^{\infty} L_{x}^{2}$ estimate, we conclude that for all $\frac{3}{2}\left(\frac{1}{2}-1 / r\right)<$ $1 / q<\frac{5}{2}\left(\frac{1}{2}-1 / r\right)$ and sufficiently small $\theta>0$ the bound

$$
\left\|\phi_{\mu, N}\right\|_{L_{t}^{q} L_{x}^{r}} \lesssim \mu^{3 / 7 q+3 / 7(1 / 2-1 / r)} N^{4 / 5 q+4 / 5(1 / 2-1 / r)}\left\|\phi_{\mu, N}\right\|_{L_{t, x}^{4}}^{\theta}\left\|\phi_{\mu, N}\right\|_{V_{+, 1}^{2}}^{1-\theta} .
$$


On the other hand, by interpolating Theorem 6 with Hölder's inequality and exploiting the null structure, we have for $1 \leqslant q, r<2,1 / q+2 / r<2$, and all sufficiently small $\theta>0$ :

$$
\begin{aligned}
& \sum_{\substack { \kappa, \kappa^{\prime} \in \mathcal{C}_{\mu^{-1}} \\
\begin{subarray}{c}{q, q^{\prime} \in \mathcal{Q}_{\mu} \\
\left|q-q^{\prime}\right| / \mu^{2}+\left|\kappa+\kappa^{\prime}\right| \approx \mu^{-1}{ \kappa , \kappa ^ { \prime } \in \mathcal { C } _ { \mu ^ { - 1 } } \\
\begin{subarray} { c } { q , q ^ { \prime } \in \mathcal { Q } _ { \mu } \\
| q - q ^ { \prime } | / \mu ^ { 2 } + | \kappa + \kappa ^ { \prime } | \approx \mu ^ { - 1 } } }\end{subarray}}\left\|\overline{R_{\kappa} P_{q} \mathcal{C}_{\ll \mu^{-1}}^{+} \psi_{\lambda_{1}, N_{1}}} R_{\kappa^{\prime}} P_{q^{\prime}} \mathcal{C}_{\ll \mu^{-1}}^{-} \varphi_{\lambda_{2}, N_{2}}\right\|_{L_{t}^{q} L_{x}^{r}} \\
& \lesssim \mu^{1 / q-1 / r+6 \theta}\left(\lambda_{1}^{-1 / 2}\left\|\psi_{\lambda_{1}, N_{1}}\right\|_{L_{t, x}^{4}} \lambda_{2}^{-1 / 2}\left\|\varphi_{\lambda_{2}, N_{2}}\right\|_{L_{t, x}^{4}}\right)^{\theta} \\
& \quad \cdot\left(\left\|\psi_{\lambda_{1}, N_{1}}\right\|_{V_{ \pm_{1}, M}^{2}}\left\|\varphi_{\lambda_{2}, N_{2}}\right\|_{V_{V_{2}, M}^{2}}\right)^{1-\theta} .
\end{aligned}
$$

Therefore, if we let $1 / r=\frac{1}{2}+\epsilon$ and $1 / q=1-\frac{9}{4} \epsilon$, then the above estimates, together with the orthogonality implied by (84), give

$$
\begin{aligned}
& \left|\int_{\mathbb{R}^{1+3}} C_{\ll \mu^{-1}} \phi_{\mu, N} \overline{\mathcal{C}_{\ll \mu^{-1}}^{+} \psi_{\lambda_{1}, N_{1}}} \mathcal{C}_{\ll \mu^{-1}}^{-} \varphi_{\lambda_{2}, N_{2}} d x d t\right| \\
& \quad \lesssim \sum_{\kappa, \kappa^{\prime} \in \mathcal{C}_{\mu^{-1}}} \sum_{\substack{q, q^{\prime} \in \mathcal{Q}_{\mu} \\
\left|q-q^{\prime}\right| / \mu^{2}+\left|\kappa+\kappa^{\prime}\right| \approx \mu^{-1}}}\left\|\phi_{\mu, N}\right\|_{L_{t}^{q^{\prime}} L_{x}^{r^{\prime}}} \\
& \quad \cdot\left\|\overline{R_{\kappa} P_{q} \mathcal{C}_{\ll \mu^{-1}}^{+} \psi_{\lambda_{1}, N_{1}}} R_{\kappa^{\prime}} P_{q^{\prime}} \mathcal{C}_{\ll \mu^{-1}}^{-} \varphi_{\lambda_{2}, N_{2}}\right\|_{L_{t}^{q} L_{x}^{r}} \\
& \quad \lesssim \mu^{7 \theta-(13 / 4) \epsilon} N^{(13 / 5) \epsilon} \mathbf{A}^{\theta}\left(\mu^{1 / 2}\left\|\phi_{\mu, N}\right\|_{V_{+, 1}^{2}}\left\|\psi_{\lambda_{1}, N_{1}}\right\|_{V_{ \pm_{1}, M}^{2}}\left\|\varphi_{\lambda_{2}, N_{2}}\right\|_{V_{ \pm_{2}, M}^{2}}\right)^{1-\theta} .
\end{aligned}
$$

Choosing $\epsilon$ and $\theta$ sufficiently small, we obtain the required bound in the case $N_{\min }=N$. The remaining cases $N_{1}=N_{\min }$ and $N_{2}=N_{\text {min }}$ are similar; the only change is to use (85) on the term with the smallest angular frequency, and control the remaining pair using the bilinear restriction estimate in Theorem 6.

Case 4: $d \gg \min \left\{\mu, \lambda_{1}, \lambda_{2}\right\}$. We start by estimating the $A_{0}$ component. As in the subcritical case, nontrivial contributions require $\mathfrak{M}_{ \pm_{1}, \pm_{2}} \approx d$. From the definition of $\mathfrak{M}_{ \pm_{1}, \pm_{2}}$ we see that either $\mathfrak{M}_{ \pm_{1}, \pm_{2}} \lesssim \min \left\{\mu, \lambda_{1}, \lambda_{2}\right\}$ or $\mathfrak{M}_{ \pm_{1}, \pm_{2}} \approx \max \{\mu$, $\left.\lambda_{1}, \lambda_{2}\right\}$. In conclusion, we must have $d \approx \max \left\{\mu, \lambda_{1}, \lambda_{2}\right\}$. If $\mu \lesssim \lambda_{1} \approx \lambda_{2}$, an application of Theorem 5 gives

$$
\begin{aligned}
\left|\int_{\mathbb{R}^{1+3}} A_{0} d x d t\right| & \leqslant\left\|C_{d} \phi_{\mu, N}\right\|_{L_{t, x}^{2}}\left\|\overline{\mathcal{C}_{\ll d}^{ \pm 1} \psi_{\lambda_{1}, N_{1}}} \mathcal{C}_{\ll d}^{ \pm_{2}} \varphi_{\lambda_{2}, N_{2}}\right\|_{L_{t, x}^{2}} \\
& \lesssim\left(\frac{d}{\lambda_{2}}\right)^{-1 / 2}\left(\frac{\mu}{\lambda_{2}}\right)^{1 / 2-\epsilon} \mu^{1 / 2}\left\|\phi_{\mu, N}\right\|_{V_{+, 1}^{2}}\left\|\psi_{\lambda_{1}, N_{1}}\right\|_{V_{ \pm_{1}, m_{1}}^{2}}\left\|\varphi_{\lambda_{2}, N_{2}}\right\|_{V_{ \pm_{2}, m_{2}}^{2}} .
\end{aligned}
$$

On the other hand, if $\mu \gg \min \left\{\lambda_{1}, \lambda_{2}\right\}$ (thus $\mu$ is essentially the largest frequency), we simply have 


$$
\begin{aligned}
\left|\int_{\mathbb{R}^{1+3}} A_{0} d x d t\right| \leqslant & \left\|C_{d} \phi_{\mu, N}\right\|_{L_{t, x}^{2}}\left\|\mathcal{C}_{\ll d}^{ \pm_{1}} \psi_{\lambda_{1}, N_{1}}\right\|_{L_{t, x}^{4}}\left\|\mathcal{C}_{\ll d}^{ \pm_{2}} \varphi_{\lambda_{2}, N_{2}}\right\|_{L_{t, x}^{4}} \\
\lesssim & \left(\frac{d}{\mu}\right)^{-1 / 2}\left(\frac{\min \left\{\lambda_{1}, \lambda_{2}\right\}}{\mu}\right)^{1 / 2} \mu^{1 / 2} \\
& \cdot\left\|\phi_{\mu, N}\right\|_{V_{+, 1}^{2}}\left\|\psi_{\lambda_{1}, N_{1}}\right\|_{V_{ \pm_{1}, m_{1}}^{2}}\left\|\varphi_{\lambda_{2}, N_{2}}\right\|_{V_{ \pm_{2}, m_{2}}^{2}} .
\end{aligned}
$$

Thus in either case we have a high-low gain, and consequently applying Lemma 5 to gain $L_{t, x}^{4}$ norms, and summing up over $d \approx \max \left\{\mu, \lambda_{1}, \lambda_{2}\right\}$ we obtain the required bound for the $A_{0}$ component.

To estimate the $A_{1}$ component, as in the subcritical case, we consider separately the cases $\min \left\{\mu, \lambda_{1}, \lambda_{2}\right\} \ll d \ll \max \left\{\mu, \lambda_{1}, \lambda_{2}\right\}$ and $d \gtrsim \max \left\{\mu, \lambda_{1}, \lambda_{2}\right\}$. In the latter case, we only require Hölder's inequality together with the (refined) $L_{t, x}^{4}$ Strichartz estimate. In particular, decomposing into cubes of size $\lambda_{\min }=$ $\min \left\{\mu, \lambda_{1}, \lambda_{2}\right\}$, we obtain

$$
\begin{aligned}
\left|\int_{\mathbb{R}^{1+3}} A_{1} d x d t\right| \lesssim & \sum_{\substack{q, q^{\prime}, q^{\prime \prime} \in \mathcal{Q}_{\lambda_{\min }} \\
\left|q-q^{\prime}+q^{\prime \prime}\right| \lesssim \lambda_{\min }}}\left\|P_{q^{\prime \prime}} \phi_{\mu, N}\right\|_{L_{t, x}^{4}}\left\|P_{q} \mathcal{C}_{d}^{ \pm_{1}} \psi_{\lambda_{1}, N_{1}}\right\|_{L_{t, x}^{2}}\left\|P_{q^{\prime}} \varphi_{\lambda_{2}, N_{2}}\right\|_{L_{t, x}^{4}} \\
\lesssim & \left(\frac{d}{\lambda_{2}}\right)^{-1 / 2}\left(\frac{\lambda_{\min }}{\lambda_{2}}\right)^{1 / 4-\epsilon}\left(\frac{\lambda_{\min }}{\mu}\right)^{1 / 4-\epsilon} \mu^{1 / 2} \\
& \cdot\left\|\phi_{\mu, N}\right\|_{V_{+, 1}^{2}}\left\|\psi_{\lambda_{1}, N_{1}}\right\|_{V_{ \pm_{1}, M}^{2}}\left\|\varphi_{\lambda_{2}, N_{1}}\right\|_{V_{ \pm_{2}, M}^{2}} .
\end{aligned}
$$

Again applying Lemma 5 and summing up over $d \gtrsim \max \left\{\mu, \lambda_{1}, \lambda_{2}\right\}$ controls the $A_{1}$ term.

We now consider the region $\min \left\{\mu, \lambda_{1}, \lambda_{2}\right\} \ll d \ll \max \left\{\mu, \lambda_{1}, \lambda_{2}\right\}$. Here we can simply observe that the bounds in the subcritical case, namely (56) and (60), imply that we have

$$
\begin{aligned}
\left|\int_{\mathbb{R}^{1+3}} A_{1} d x d t\right| \lesssim & \left(\frac{d}{\min \left\{\mu, \lambda_{1}, \lambda_{2}\right\}}\right)^{-1 / 2}\left(\frac{\min \left\{\mu, \lambda_{1}, \lambda_{2}\right\}}{\max \left\{\mu, \lambda_{1}, \lambda_{2}\right\}}\right)^{1 / 4} \mu^{1 / 2} \\
& \cdot\left\|\phi_{\mu, N}\right\|_{V_{+, 1}^{2}}\left\|\psi_{\lambda_{1}, N_{1}}\right\|_{V_{ \pm_{1}, M}^{2}}\left\|\varphi_{\lambda_{2}, N_{1}}\right\|_{V_{ \pm_{2}, M}^{2}}
\end{aligned}
$$

Again applying Lemma 5 and summing up over

$$
\min \left\{\mu, \lambda_{1}, \lambda_{2}\right\} \ll d \ll \max \left\{\mu, \lambda_{1}, \lambda_{2}\right\}
$$

we deduce the required bound for the $A_{1}$ term. An identical argument bounds the $A_{2}$ term. 
7.2. Proof of Theorem 9. Similarly to Section 6.2, the first step is to obtain frequency localized versions of the required bounds. Let $\lambda_{\min }=\min \left\{\mu, \lambda_{1}, \lambda_{2}\right\}$ and $\lambda_{\max }=\max \left\{\mu, \lambda_{1}, \lambda_{2}\right\}$. Our aim is to show that, if $\varrho>0$ is sufficiently small, $1 / a=\frac{1}{2}+\varrho / 16$, and $b=2\left(1 / a-\frac{1}{2}\right)$, then there exists $0<\theta_{1}<\frac{1}{4}$ such that for all $0 \leqslant \theta \leqslant \theta_{1}$ we have for the Dirac Duhamel term, the bounds

$$
\begin{aligned}
& \left\|P_{\lambda_{1}} H_{N_{1}} \Pi_{ \pm_{1}} \mathcal{I}^{ \pm_{1}, M}\left(\phi_{\mu, N} \gamma^{0} \Pi_{ \pm_{2}} \varphi_{\lambda_{2}, N_{2}}\right)\right\|_{V_{ \pm_{1}, M}^{2}} \\
& \lesssim\left(\min \left\{N, N_{2}\right\}\right)^{\varrho}\left(\frac{\lambda_{\min }}{\lambda_{\max }}\right)^{\varrho / 100}\left(\left\|\phi_{\mu, N}\right\|_{L_{t, x}^{4}} \lambda_{2}^{-1 / 2}\left\|\varphi_{\lambda_{2}, N_{2}}\right\|_{L_{t, x}^{4}}\right)^{\theta} \\
& \quad \cdot\left(\mu^{1 / 2}\left\|\phi_{\mu, N}\right\|_{V_{+, 1}^{2}}\left\|\varphi_{N_{2}}\right\|_{F_{\lambda_{2}}^{ \pm_{2}, M}}\right)^{1-\theta}
\end{aligned}
$$

and

$$
\begin{aligned}
\left\|H_{N_{1}} \Pi_{ \pm_{1}} \mathcal{I}^{ \pm_{1}, M}\left(\phi_{\mu, N} \gamma^{0} \Pi_{ \pm_{2}} \varphi_{\lambda_{2}, N_{2}}\right)\right\|_{Y_{\lambda_{1}}^{ \pm 1}, M} \\
\lesssim\left(\min \left\{N, N_{2}\right\}\right)^{\varrho}\left(\frac{\lambda_{\min }}{\lambda_{\max }}\right)^{\varrho / 100}\left(\left\|\phi_{\mu, N}\right\|_{L_{t, x}^{4}} \lambda_{2}^{-1 / 2}\left\|\varphi_{\lambda_{2}, N_{2}}\right\|_{L_{t, x}^{4}}\right)^{\theta} \\
\quad \cdot\left(\mu^{1 / 2}\left\|\phi_{\mu, N}\right\|_{V_{+, 1}^{2}}\left\|\varphi_{\lambda_{2}, N_{2}}\right\|_{V_{ \pm_{2}, M}^{2}}\right)^{1-\theta},
\end{aligned}
$$

while for the wave Duhamel term, we have

$$
\begin{aligned}
& \mu^{-1 / 2}\left\|P_{\mu} H_{N} \mathcal{I}^{+, 1}\left(\overline{\Pi_{ \pm_{1}} \psi_{\lambda_{1}, N_{1}}} \Pi_{ \pm_{2}} \varphi_{\lambda_{2}, N_{2}}\right)\right\|_{V_{+, 1}^{2}} \\
& \lesssim\left(\min \left\{N_{1}, N_{2}\right\}\right)^{\varrho}\left(\frac{\lambda_{\min }}{\lambda_{\max }}\right)^{\varrho / 100}\left(\lambda_{1}^{-1 / 2}\left\|\psi_{\lambda_{1}, N_{1}}\right\|_{L_{t, x}^{4}} \lambda_{2}^{-1 / 2}\left\|\varphi_{\lambda_{2}, N_{2}}\right\|_{L_{t, x}^{4}}\right)^{\theta} \\
& \cdot\left(\left\|\psi_{N_{1}}\right\|_{F_{\lambda_{1}}^{ \pm_{1}, M}}\left\|\varphi_{N_{2}}\right\|_{F_{\lambda_{2}}^{ \pm_{2}, M}}\right)^{1-\theta} .
\end{aligned}
$$

Assuming bounds (86), (87), and (88) for the moment, the estimates in Theorem 9 are a consequence of a straightforward summation argument. Fix $\sigma>0$. As in the subcritical case, it is enough to consider the case $s=0$ by using that, due to the convolution constraint, we always have $\lambda_{1}^{s} \lesssim\left(\max \left\{\mu, \lambda_{2}\right\}\right)^{s}$. Summing up (86) with $\varrho=\sigma / 2$ over angular frequencies $N_{1}$ gives $0<\theta_{0}<\frac{1}{2}$ such that for all $0<\theta<\theta_{0}$ we have

$$
\begin{aligned}
\left(\sum_{N_{1} \in 2^{\mathbb{N}}} N_{1}^{2 \sigma}\left\|P_{\lambda_{1}} H_{N_{1}} \Pi_{ \pm_{1}} \mathcal{I}_{M}^{ \pm 1}\left(\phi_{\mu} \gamma^{0} \Pi_{ \pm_{2}} \varphi_{\lambda_{2}}\right)\right\|_{V_{ \pm_{1}, M}^{2}}^{2}\right)^{1 / 2} & \\
\lesssim & \left(\frac{\lambda_{\min }}{\lambda_{\max }}\right)^{\sigma / 200}\left(\left\|\phi_{\mu}\right\|_{\mathbf{D}^{0, \sigma}}\left\|\varphi_{\lambda_{2}}\right\|_{\mathbf{D}^{-1 / 2, \sigma}}\right)^{\theta} \\
& \cdot\left[\mu^{1 / 2}\left(\sum_{N \in 2^{\mathbb{N}}} N^{2 \sigma}\left\|\phi_{\mu, N}\right\|_{V_{+, 1}^{2}}^{2}\right)^{1 / 2}\left(\sum_{N_{2} \in 2^{\mathbb{N}}} N_{2}^{2 \sigma}\left\|\varphi_{N_{2}}\right\|_{F_{\lambda_{2}}^{ \pm 2, M}}^{2}\right)^{1 / 2}\right]^{1-\theta} .
\end{aligned}
$$


Note that for $1 / q+1 / r=\frac{1}{2}$ and $\epsilon>0$ we have the elementary inequality

$$
\begin{aligned}
& \left\|\left(\sum_{\substack{\mu, \lambda_{2} \in 2^{\mathbb{N}} \\
\mu+\lambda_{2} \approx \lambda_{1}}}\left(\frac{\min \left\{\mu, \lambda_{2}\right\}}{\lambda_{1}}\right)^{\epsilon} a_{\mu} b_{\lambda_{2}}\right)_{\lambda_{1} \in 2^{\mathbb{N}}}\right\|_{\ell^{2}}+\left\|\left(\sum_{\substack{\mu, \lambda_{2} \in 2^{\mathbb{N}} \\
\mu \approx \lambda_{2} \gtrsim \lambda_{1}}}\left(\frac{\lambda_{1}}{\lambda_{2}}\right)^{\epsilon} a_{\mu} b_{\lambda_{2}}\right)_{\lambda_{1} \in 2^{\mathbb{N}}}\right\|_{\ell^{2}} \| \\
& \quad \lesssim\left\|\left(a_{\mu}\right)_{\mu \in 2^{\mathbb{N}}}\right\|_{\ell q}\left\|\left(b_{\lambda_{2}}\right)_{\lambda_{2} \in 2^{\mathbb{N}}}\right\|_{\ell^{r}} .
\end{aligned}
$$

Thus summing up (89) over spatial frequencies, and assuming that $0<\theta<\frac{1}{4}$, we deduce bound (71). An identical argument using (87) gives the $\mathbf{Y}_{ \pm_{1}, M}^{s}$ bound (72). Similarly the bound for $\phi$ follows from (88).

We now turn to the proof of estimates (86), (87), and (88). It is enough to consider the case $\theta=\theta_{1}$, as the $L_{t, x}^{4}$ terms are dominated by the corresponding $V^{2}$ norms. Bounds (86) and (88) follow directly from Theorem 10 together with (24). On the other hand, the argument used to obtain (87) is slightly more involved. We first note that, from [6, (8-38)-(8-40)], we have the bound

$$
\begin{aligned}
& d^{2 / 3}\left(\frac{\min \left\{d, \lambda_{1}\right\}}{\lambda_{1}}\right)^{1-2 / 3}\left\|P_{\lambda_{1}} H_{N_{1}} \mathcal{C}_{d}^{ \pm_{1}} \mathcal{I}^{ \pm_{1}, M}\left(\phi_{\mu, N} \gamma^{0} \Pi_{ \pm_{2}} \varphi_{\lambda_{2}, N_{2}}\right)\right\|_{L_{t}^{3 / 2} L_{x}^{2}} \\
& \lesssim \min \left\{N, N_{2}\right\}\left(\frac{\lambda_{\max }}{\lambda_{\min }}\right)^{1 / 3} \mu^{1 / 2}\left\|\phi_{\mu, N}\right\|_{V_{+, 1}^{2}}\left\|\psi_{\lambda_{1}, N_{1}}\right\|_{V_{ \pm_{2}, M}^{2}} .
\end{aligned}
$$

On the other hand, if $\mu \lesssim \lambda_{2}$, then an application of (79) gives

$$
\begin{aligned}
& d^{1 / 2} \| P_{\lambda_{1}} H_{N_{1}} \mathcal{C}_{d}^{ \pm_{1}} \mathcal{I}^{ \pm_{1}, M}\left(\phi_{\mu, N} \gamma^{0} \Pi_{ \pm_{2}} \varphi_{\lambda_{2}, N_{2}}\right) \|_{L_{t, x}^{2}} \\
& \lesssim\left\|P_{\lambda_{1}} H_{N_{1}} \mathcal{C}_{d}^{ \pm_{1}} \mathcal{I}^{ \pm_{1}, M}\left(\phi_{\mu, N} \gamma^{0} \Pi_{ \pm_{2}} \varphi_{\lambda_{2}, N_{2}}\right)\right\|_{V_{ \pm_{1}, M}^{2}} \\
& \lesssim\left(\min \left\{N, N_{2}\right\}\right)^{\varrho / 2}\left(\frac{\mu}{\lambda_{2}}\right)^{1 / 10}\left(\left\|\phi_{\mu}\right\|_{L_{t, x}^{4}} \lambda_{2}^{-1 / 2}\left\|\varphi_{\lambda_{2}}\right\|_{L_{t, x}^{4}}\right)^{\theta_{0}} \\
& \quad \cdot\left(\mu^{1 / 2}\left\|\phi_{\mu}\right\|_{V_{+, 1}^{2}}\left\|\varphi_{\lambda_{2}}\right\|_{V_{ \pm_{2}, M}^{2}}\right)^{1-\theta_{0}} .
\end{aligned}
$$

Hence (87) in the region $\mu \lesssim \lambda_{2}$ follows by interpolating between (90) and (91) and using the conditions $1 / a=\frac{1}{2}+\varrho / 16$ and $b=2\left(1 / a-\frac{1}{2}\right)$. The case $\mu \gg \lambda_{2}$ and $N \leqslant N_{2}$ follows from a similar argument using (80) and (81).

It remains to consider the case $\mu \gg \lambda_{2}$ and $N_{2} \leqslant N$. For this frequency interaction, Theorem 10 requires a $Y_{\lambda_{2}}^{ \pm, M}$ norm on the right-hand side. Thus, as our goal is to obtain a bound only using the $V_{ \pm, M}^{2}$ norms, we have to work a 
little harder. We start by writing the product as

$$
\begin{aligned}
\phi_{\mu, N} \gamma^{0} \Pi_{ \pm_{2}} \varphi_{\lambda_{2}, N_{2}}= & \left(\phi_{\mu, N} \gamma^{0} \Pi_{ \pm_{2}} \varphi_{\lambda_{2}, N_{2}}-\sum_{d^{\prime} \lesssim \lambda_{2}} C_{\leqslant d^{\prime}}^{ \pm A_{1}, M}\left(C_{\leqslant d^{\prime}} \phi_{\mu, N} \gamma^{0} \mathcal{C}_{d^{\prime}}^{ \pm_{2}} \varphi_{\lambda_{2}, N_{2}}\right)\right) \\
& +\sum_{d^{\prime} \lesssim \lambda_{2}} C_{\leqslant d^{\prime}}^{ \pm_{1}, M}\left(C_{\leqslant d^{\prime}} \phi_{\mu, N} \gamma^{0} \mathcal{C}_{d^{\prime}}^{ \pm_{2}} \varphi_{\lambda_{2}, N_{2}}\right) .
\end{aligned}
$$

The first term can be bounded by adapting the argument used in the previous cases as here (80) in Theorem 10 gives a bound without using the $Y_{\lambda_{2}}^{ \pm, M}$ norm. More precisely, letting $\beta=\left(d^{\prime} / \lambda_{2}\right)^{1 / 2}$ and exploiting the null structure together with the now familiar modulation bounds, we have

$$
\begin{aligned}
& d^{2 / 3}\left(\frac{d}{\lambda_{1}}\right)^{1-2 / 3}\left\|P_{\lambda_{1}} H_{N_{1}} \mathcal{C}_{d}^{ \pm} \mathcal{I}^{ \pm_{1}, M}\left(\sum_{d^{\prime} \lesssim \lambda_{2}} C_{\leqslant d^{\prime}}^{ \pm_{1}, M}\left(C_{\leqslant d^{\prime}} \phi_{\mu, N} \gamma^{0} \mathcal{C}_{d^{\prime}}^{ \pm_{2}} \varphi_{\lambda_{2}, N_{2}}\right)\right)\right\|_{L_{t}^{3 / 2} L_{x}^{2}}\left\|\sum_{d^{\prime} \lesssim \lambda_{2}}\right\|\left(\sum_{\substack{\kappa, \kappa^{\prime}, k^{\prime \prime} \in \mathcal{C}_{\beta} \\
\left| \pm_{1} \kappa- \pm_{2} \kappa^{\prime}\right|,\left|\kappa-\kappa^{\prime \prime}\right| \lesssim \beta}}\left\|R_{\kappa}\left(C_{\leqslant d^{\prime}} R_{\kappa^{\prime \prime}} \phi_{\mu, N} \gamma^{0} R_{\kappa^{\prime}} \mathcal{C}_{d^{\prime}}^{ \pm_{2}} \varphi_{\lambda_{2}, N_{2}}\right)\right\|_{L_{x}^{2}}^{2}\right)^{1 / 2} \|_{L_{t}^{3 / 2}} \\
& \quad \lesssim \mu^{-1 / 3} \sum_{d^{\prime} \lesssim \lambda_{2}} \beta\left(\sum_{\kappa^{\prime \prime} \in \mathcal{C}_{\beta}}\left\|C_{\leqslant d^{\prime}} R_{\kappa^{\prime \prime}} \phi_{\mu, N}\right\|_{L_{t, x}^{4}}^{2}\right)^{1 / 2}\left\|\varphi_{\lambda_{2}, N_{2}}\right\|_{L_{t}^{12 / 5} L_{x}^{4}} \\
& \lesssim N_{2}\left(\frac{\lambda_{2}}{\mu}\right)^{1 / 3} \mu^{1 / 2}\left\|\phi_{\mu, N}\right\|_{V_{+, 1}^{2}}\left\|\varphi_{\lambda_{2}, N_{2}}\right\|_{V_{ \pm_{2}, M}^{2}} .
\end{aligned}
$$

Together with (90), we deduce that

$$
\begin{gathered}
d^{2 / 3}\left(\frac{\min \left\{d, \lambda_{1}\right\}}{\lambda_{1}}\right)^{1-2 / 3} \| P_{\lambda_{1}} H_{N_{1}} \mathcal{C}_{d}^{ \pm_{1}} \mathcal{I}^{ \pm_{1}, M}\left(\phi_{\mu, N} \gamma^{0} \varphi_{\lambda_{2}, N_{2}}\right. \\
\left.\quad-\sum_{d^{\prime} \lesssim \lambda_{2}} C_{\leqslant d^{\prime}}^{ \pm_{1}, M}\left(C_{\leqslant d^{\prime}} \phi_{\mu, N} \gamma^{0} \mathcal{C}_{d^{\prime}}^{ \pm_{2}} \varphi_{\lambda_{2}, N_{2}}\right)\right) \|_{L_{t}^{3 / 2} L_{x}^{2}} \\
\lesssim N\left(\frac{\mu}{\lambda_{2}}\right)^{1 / 3} \mu^{1 / 2}\left\|\phi_{\mu, N}\right\|_{V_{+, 1}^{2}}\left\|\varphi_{\lambda_{2}, N_{2}}\right\|_{V_{ \pm_{2}, M}^{2}} .
\end{gathered}
$$

Applying $L^{p}$ interpolation together with (80) and arguing as previously, we deduce that

$$
\begin{aligned}
& \left\|H_{N_{1}} \Pi_{ \pm_{1}} \mathcal{I}^{ \pm_{1}, M}\left(\phi_{\mu, N} \gamma^{0} \varphi_{\lambda_{2}, N_{2}}-\sum_{d^{\prime} \lesssim \lambda_{2}} C_{\leqslant d^{\prime}}^{ \pm_{1}, M}\left(C_{\leqslant d^{\prime}} \phi_{\mu, N} \gamma^{0} \mathcal{C}_{d^{\prime}}^{ \pm 2} \varphi_{\lambda_{2}, N_{2}}\right)\right)\right\|_{Y_{\lambda_{1}}^{ \pm}, M} \\
& \lesssim N^{\varrho}\left(\frac{\lambda_{2}}{\mu}\right)^{\varrho / 100}\left(\left\|\phi_{\mu, N}\right\|_{L_{t, x}^{4}} \lambda_{2}^{-1 / 2}\left\|\varphi_{\lambda_{2}, N_{2}}\right\|_{L_{t, x}^{4}}\right)^{\theta}\left(\mu^{1 / 2}\left\|\phi_{\mu, N}\right\|_{V_{+, 1}^{2}}\left\|\varphi_{\lambda_{2}, N_{2}}\right\|_{V_{ \pm_{2}, M}^{2}}\right)^{1-\theta} .
\end{aligned}
$$


Therefore, it only remains to bound the second term in (92), but this follows by taking $1 / r=1-2\left(1 / a-\frac{1}{2}\right)$ in $(70)$.

\section{Acknowledgements}

The authors acknowledge financial support by the DFG through the CRC 1283 'Taming uncertainty and profiting from randomness and low regularity in analysis, stochastics and their applications'. The authors would also like to thank the referees for their careful reading of the manuscript.

\section{References}

[1] I. Bejenaru and S. Herr, 'On global well-posedness and scattering for the massive DiracKlein-Gordon system', J. Eur. Math. Soc. (JEMS) 19(8) (2017), 2445-2467. MR 3668064.

[2] J. D. Bjorken and S. D. Drell, Relativistic Quantum Mechanics (McGraw-Hill Book Co., New York-Toronto-London, 1964), MR 0187641.

[3] J. Bourgain, Global Solutions of Nonlinear Schrödinger Equations, American Mathematical Society Colloquium Publications, 46 (American Mathematical Society, Providence, RI, 1999). MR 1691575.

[4] T. Candy, 'Multiscale bilinear restriction estimates for general phases', Preprint, 2017, arXiv:1707.08944 [math.CA].

[5] T. Candy and S. Herr, 'On the Majorana condition for nonlinear Dirac systems', Ann. Inst. H. Poincaré Anal. Non Linéaire (2018), doi:10.1016/j.anihpc.2018.02.001.

[6] T. Candy and S. Herr, 'Transference of bilinear restriction estimates to quadratic variation norms and the Dirac-Klein-Gordon system', Anal. PDE 11(5) (2018), 1171-1240. MR 3785603.

[7] Y. Cho and S. Lee, 'Strichartz estimates in spherical coordinates', Indiana Univ. Math. J. 62(3) (2013), 991-1020. MR 3164853.

[8] J. Colliander, M. Keel, G. Staffilani, H. Takaoka and T. Tao, 'Global well-posedness and scattering for the energy-critical nonlinear Schrödinger equation in $\mathbb{R}^{3}$, Ann. of Math. (2) 167(3) (2008), 767-865. MR 2415387.

[9] P. D'Ancona, D. Foschi and S. Selberg, 'Null structure and almost optimal local regularity for the Dirac-Klein-Gordon system', J. Eur. Math. Soc. (JEMS) 9(4) (2007), 877-899. MR 2341835.

[10] B. Dodson and P. Smith, 'A controlling norm for energy-critical Schrödinger maps', Trans. Amer. Math. Soc. 367(10) (2015), 7193-7220. MR 3378828.

[11] M. J. Esteban, V. Georgiev and E. Séré, 'Stationary solutions of the Maxwell-Dirac and the Klein-Gordon-Dirac equations', Calc. Var. Partial Differ. Equ. 4(3) (1996), 265-281. MR 1386737.

[12] M. Hadac, S. Herr and H. Koch, 'Well-posedness and scattering for the KP-II equation in a critical space', Ann. Inst. H. Poincaré Anal. Non Linéaire 26(3) (2009), 917-941. MR 2526409.

[13] C. E. Kenig, Lectures on the Energy Critical Nonlinear Wave Equation, CBMS Regional Conference Series in Mathematics, 122 (American Mathematical Society, Providence, RI, 2015), Published for the Conference Board of the Mathematical Sciences, Washington, DC. MR 3328916. 
[14] R. Killip and M. Vişan, 'Nonlinear Schrödinger equations at critical regularity', in Evolution Equations, Clay Math. Proc., 17 (American Mathematical Society, Providence, RI, 2013), 325-437. MR 3098643.

[15] H. Koch and D. Tataru, 'Dispersive estimates for principally normal pseudodifferential operators', Comm. Pure Appl. Math. 58(2) (2005), 217-284. MR 2094851.

[16] H. Koch, D. Tataru and M. Vişan, Dispersive Equations and Nonlinear Waves. Generalized Korteweg-de Vries, Nonlinear Schrödinger, Wave and Schrödinger Maps, (Birkhäuser/Springer, Basel, 2014), (English).

[17] S. Machihara, K. Nakanishi and T. Ozawa, 'Small global solutions and the nonrelativistic limit for the nonlinear Dirac equation', Rev. Mat. Iberoam. 19(1) (2003), 179-194.

MR 1993419.

[18] S.-J. Oh and D. Tataru, 'Global well-posedness and scattering of the $(4+1)$-dimensional Maxwell-Klein-Gordon equation', Invent. Math. 205(3) (2016), 781-877. MR 3539926.

[19] S.-J. Oh and D. Tataru, 'Local well-posedness of the $(4+1)$-dimensional Maxwell-KleinGordon equation at energy regularity', Ann. PDE 2(1) (2016), 70. Art. 2, MR 3462105.

[20] J. Sterbenz, 'Global regularity and scattering for general non-linear wave equations. II. $(4+1)$ dimensional Yang-Mills equations in the Lorentz gauge', Amer. J. Math. 129(3) (2007), 611-664. MR 2325100.

[21] J. Sterbenz and D. Tataru, 'Energy dispersed large data wave maps in $2+1$ dimensions', Comm. Math. Phys. 298(1) (2010), 139-230. MR 2657817.

[22] J. Sterbenz and D. Tataru, 'Regularity of wave-maps in dimension 2+1', Comm. Math. Phys. 298(1) (2010), 231-264.

[23] B. Thaller, The Dirac Equation, Texts and Monographs in Physics (Springer, Berlin, 1992). MR 1219537.

[24] X. Wang, 'On global existence of 3D charge critical Dirac-Klein-Gordon system', Int. Math. Res. Not. IMRN 2015(21) (2015), 10801-10846. MR 3456028. 